\title{
Higher-order spin effects in the amplitude and phase of gravitational waveforms emitted by inspiraling compact binaries: Ready-to-use gravitational waveforms
}

\author{
K. G. Arun, ${ }^{1,2, *}$ Alessandra Buonanno, ${ }^{3, \text {, }}$ Guillaume Faye, ${ }^{1, \text { I }}$ and Evan Ochsner ${ }^{3}$, 鸟 \\ ${ }^{1}$ Institut d'Astrophysique de Paris, UMR 7095-CNRS, \\ Université Pierre et Marie Curie, $98^{\text {bis }}$ boulevard Arago, 75014 Paris, France \\ ${ }^{2}$ LAL, Université Paris-Sud, IN2P3/CNRS, Orsay, France \\ ${ }^{3}$ Maryland Center for Fundamental Physics, Department of Physics, \\ University of Maryland, College Park, MD 20742, USA
}

\begin{abstract}
We provide ready-to-use time-domain gravitational waveforms for spinning compact binaries with precession effects through 1.5PN order in amplitude and compute their mode decomposition using spin-weighted -2 spherical harmonics. In the presence of precession, the gravitational-wave modes $(\ell, m)$ contain harmonics originating from combinations of the orbital frequency and precession frequencies. We find that the gravitational radiation from binary systems with large mass asymmetry and large inclination angle can be distributed among several modes. For example, during the last stages of inspiral, for some maximally spinning configurations, the amplitude of the $(2,0)$ and $(2,1)$ modes can be comparable to the amplitude of the $(2,2)$ mode. If the mass ratio is not too extreme, the $\ell=3$ and $\ell=4$ modes are generally one or two orders of magnitude smaller than the $\ell=2$ modes. Restricting ourselves to spinning, non-precessing compact binaries, we apply the stationaryphase approximation and derive the frequency-domain gravitational waveforms including spin-orbit and spin(1)-spin(2) effects through $1.5 \mathrm{PN}$ and $2 \mathrm{PN}$ order respectively in amplitude, and $2.5 \mathrm{PN}$ order in phase. Since spin effects in the amplitude through $2 \mathrm{PN}$ order affect only the first and second harmonics of the orbital phase, they do not extend the mass reach of gravitational-wave detectors. However, they can interfere with other harmonics and lower or raise the signal-to-noise ratio depending on the spin orientation. These ready-to-use waveforms could be employed in the data-analysis of the spinning, inspiraling binaries as well as in comparison studies at the interface between analytical and numerical relativity.
\end{abstract}

\section{INTRODUCTION}

Coalescing compact binaries made of neutron stars (NS) and/or black holes (BH) can produce gravitational waves (GW) strong enough to be detected by ground-based interferometers, such as LIGO [1], Virgo [2] and GEO [3], operating in the frequency range $10-10^{4} \mathrm{~Hz}$. Moreover, supermassive BH binaries could be observed at lower frequencies $10^{-5}-10^{-1} \mathrm{~Hz}$ and up to cosmological distances by the proposed laser space-based antenna LISA [4]. For detection purposes, matched filtering is applied to noisy data in order to extract any signals that match members of the template bank [5] 7 .

Gravitational waves produced during the long inspiral phase can accurately be modeled by the post-Newtonian (PN) approximation to general relativity [8]. As the BHs approach each other and their velocities increase, the PN expansion is expected to become less and less reliable. Late in the evolution, non-perturbative information contained in numerical-relativity (NR) simulations and PN-resummed methods [9], as well as perturbation theory need to be taken into account in building analytical templates for inspiral, merger, and ringdown. In this paper, we shall limit the discussion to the inspiral phase of coalescing BHs.

In constructing templates for detecting inspiraling signals, it is recommended to account for all physical effects which contribute significantly to the gravitational waveform. Those produced by the spins of the binary constituents are among the most important ones, especially for asymmetric compact binaries [10], such as NS-BH binaries [11], and BH-BH binaries with component masses $\left(m_{1}, m_{2}\right) \in[5,15] M_{\odot} \times[1,5] M_{\odot}$. For detecting such systems, one may be able to employ phenomenological methods which capture the essential features of spinning, precessing waveforms [12]. However, parameter extraction [13 15] would warrant the inclusion of as much information about the spins of the binaries as possible, so that one should employ physical templates [16 18] at the highest PN order for this purpose.

\footnotetext{
*Electronic address: arun@iap.fr

$\dagger$ Electronic address: buonanno@umd.edu

$\ddagger$ Electronic address: faye@iap.fr

$\S$ Electronic address: evano@umd.edu
} 
For non-spinning compact binaries, the GW phase evolution has been computed through 3.5PN order 19 23 and the $h_{+}$and $h_{\times}$polarizations are available through 3PN order [24 28]. For spinning, precessing binaries, the GW phase evolution is known through 2.5PN order [29, 30] for spin-orbit couplings, and through 2PN order [31 for spin-spin couplings ( $\operatorname{spin}(1)$-spin(1) and $\operatorname{spin}(2)$-spin(2) contributions have been obtained in Refs. 32, 33]). Spin-orbit and spin(1)-spin(2) effects in the $h_{+}$and $h_{\times}$polarizations were computed through $1.5 \mathrm{PN}$ and $2 \mathrm{PN}$ order, respectively, in Refs. [34, 35]. ${ }^{1}$ More recently, the spin(1)-spin(2) contributions at 3PN order in the conservative two-body dynamics were found employing either effective-field theory techniques [37 40] or the Hamiltonian formalism of Arnowitt, Deser and Misner [41 43]. Now, spin(1)-spin(1) and spin(2)-spin(2) effects at 3PN order in the conservative twobody dynamics are also available 44. For including those higher-order spin effects in the GW phase evolution and polarizations, the results [39 41, 43, 44] need to be extended to the non-conservative dynamics, notably to the GW energy flux.

The importance of using templates that have amplitude corrections beyond the leading PN order (henceforth referred to as Newtonian approximation ${ }^{2}$ ) was emphasized by different authors in the context of ground-based [45[48] and space-based detectors [49 [55], both for detection and parameter estimation. So far, the effect of spins and precession on parameter estimation was studied in Refs. 15, 56 [58], but those studies were limited to non-spinning and Newtonian GW polarizations 24 26].

In this paper we provide ready-to-use $h_{+}$and $h_{\times}$polarizations in time domain for spinning, precessing binaries through 1.5PN order. The actual computation of the gravitational waveform $h_{i j}$ through $1.5 \mathrm{PN}$ order was done by Kidder [34], as well as Will and Wiseman [35], but the ready-to-use $h_{+}$and $h_{\times}$polarizations at 1.5PN order were only written explicitly for strictly circular orbits for which spins are aligned with the orbital angular momentum. Recently, Ref. [59] has obtained the time-domain GW polarizations for generic orbits through 1.5PN order in the binary's comoving frame. The $h_{+}$and $h_{\times}$polarizations derived in the present paper for spinning, precessing binaries through 1.5PN order reduces to that of Refs. [34, 35] in the aligned case except for a few typographical errors which we correct.

In view of future studies at the interface between analytical and numerical relativity [60 71] we decompose the timedomain $h_{+}$and $h_{\times}$polarizations in spin-weighted -2 spherical harmonics and compute the modes, $h_{\ell m}$, to $1.5 \mathrm{PN}$ order. We then consider spinning, non-precessing binaries for which we derive the Fourier domain representation of the generated gravitational waveform within the stationary phase approximation (SPA). We provide a very compact way of writing the Fourier transforms of $h_{+}$and $h_{\times}$which can readily be used for data analysis, for comparisons with numerical simulations, or for building analytical frequency-domain templates including inspiral, merger and ringdown [72, 73]. The impact of spinning, precessing waveforms for parameter estimation will be investigated in a future paper.

The remainder of the paper is organized in the following way. In Sec. III we draw the source and detector frames, and introduce conventions and notations. In Sec. III we provide ready-to-use $h_{+}$and $h_{\times}$polarizations in time domain for nearly circular orbits. The polarization modes $h_{\ell m}$ with respect to the spin-weighted -2 spherical harmonics are derived in Sec. IV] The features of the modes when spins are present is then discussed in Sec. V. Section VI focuses on spinning, non-precessing binaries. We compute there the Fourier domain waveforms with spin effects through 2PN order in the amplitude and 2.5PN order in the phase before discussing the main features caused by higher harmonics. Finally, we summarize in Sec. VII our main conclusions. Appendices $\mathrm{A}$ and B present the GW polarizations and modes for precessing binaries on nearly circular orbits through 1.5PN order for generic inclination angles, whereas Appendix [C shows the PN coefficients of the center-of-mass energy and radiative energy flux for non-precessing, spinning binaries. Appendix D gives explicitly the frequency domain amplitude coefficients with non-spin terms to $2.5 \mathrm{PN}$ and spin terms to $2 \mathrm{PN}$ order.

\section{SOURCE FRAME, POLARIZATION AND PARAMETER CONVENTIONS}

To obtain the GW polarizations, it is useful to express the gravitational strain tensor, $h_{i j}$, in an appropriate source

frame. Next, one specifies an orthonormal polarization triad composed of the direction of propagation $\hat{\mathbf{N}}$ and two

\footnotetext{
${ }^{1}$ Note that spin-orbit effects through $2 \mathrm{PN}$ order in the $h_{+}$and $h_{\times}$polarizations were calculated in Ref. [36]. However, Ref. [30] pointed out that a few multipole moments were computed incorrectly there.

2 Note that the leading PN order in the polarization amplitude is proportional to $1 / c^{4}$ when one turns the fundamental constants on. However, being the leading term in a PN expansion, it is has become common to call it Newtonian.
} 
polarization vectors $\hat{\mathbf{P}}$ and $\hat{\mathbf{Q}}$ which are used to construct the GW polarizations from the strain tensor [74]:

$$
\begin{aligned}
& h_{+}=\frac{1}{2}\left(\hat{P}^{i} \hat{P}^{j}-\hat{Q}^{i} \hat{Q}^{j}\right) h_{i j}, \\
& h_{\times}=\frac{1}{2}\left(\hat{P}^{i} \hat{Q}^{j}+\hat{Q}^{i} \hat{P}^{j}\right) h_{i j} .
\end{aligned}
$$

The gravitational strain measured by a detector is then given by

$$
h_{\text {strain }}(t)=F_{+} h_{+}(t)+F_{\times} h_{\times}(t),
$$

where $F_{+}$and $F_{\times}$are the antenna response functions that describe the detector's sensitivity to the two different polarizations. For laser interferometers with arms at a right angle, such as the LIGO and Virgo detectors, the antenna response functions for a GW coming from the sky location $(\bar{\theta}, \bar{\phi})$ in the spherical coordinate grid built from the arm basis, with polarization angle $\bar{\psi}$, are 74 ]

$$
\begin{aligned}
& F_{+}=\frac{1}{2}\left(1+\cos ^{2} \bar{\theta}\right) \cos 2 \bar{\phi} \cos 2 \bar{\psi}-\cos \bar{\theta} \sin 2 \bar{\phi} \sin 2 \bar{\psi}, \\
& F_{\times}=\frac{1}{2}\left(1+\cos ^{2} \bar{\theta}\right) \cos 2 \bar{\phi} \sin 2 \bar{\psi}+\cos \bar{\theta} \sin 2 \bar{\phi} \cos 2 \bar{\psi}
\end{aligned}
$$

Note that the strain measured in a given instrument, $h_{\text {strain }}(t)$, is the same regardless of convention, whereas the wave polarizations depend on the choice of polarization vectors. Different choices of $\hat{\mathbf{P}}$ and $\hat{\mathbf{Q}}$ give different polarizations, but there is a compensating rotation of the polarization angle $\bar{\psi}$ so that $h_{\text {strain }}(t)$ is unchanged ${ }^{3}$. Here, we follow the convention of Refs. 25, 35], in which

$$
\hat{\mathbf{P}}=\frac{\hat{\mathbf{N}} \times \mathbf{J}_{0}}{\left|\hat{\mathbf{N}} \times \mathbf{J}_{0}\right|}, \quad \hat{\mathbf{Q}}=\hat{\mathbf{N}} \times \hat{\mathbf{P}},
$$

where $\mathbf{J}_{0}$ is the unit vector along the initial total angular momentum of the binary. In the absence of precession, the Newtonian orbital angular momentum $\mathbf{L}_{N}=\mu \mathbf{r} \times \mathbf{v}$ (with $\mathbf{r}, \mathbf{v}$, and $\mu$ being the binary separation vector, velocity, and reduced mass, respectively) is parallel to $\mathbf{J}_{0}$. In this case, $\hat{\mathbf{P}}$ coincides with the ascending node where the orbital separation vector crosses the plane of the sky from below. In the presence of precession, $\hat{\mathbf{P}}$ is still defined as $\hat{\mathbf{N}} \times \mathbf{J}_{0} /\left|\hat{\mathbf{N}} \times \mathbf{J}_{0}\right|$, but it is not in general the point where the orbital separation vector ascends through the plane of the sky. 4

For our source frame, we construct an adapted orthonormal basis $(\hat{\mathbf{x}}, \hat{\mathbf{y}}, \hat{\mathbf{z}})$ (see Fig. 11). We take the $z$-axis to be along $\mathbf{J}_{0}$ and the direction of GW propagation, $\hat{\mathbf{N}}$, to lie in the $x-z$ plane, tilted by an angle $\theta$ from the $z$-axis towards the $x$-axis. We describe the direction of the Newtonian orbital angular momentum with the spherical coordinate angles $(\iota, \alpha)$, where $\iota$ denotes the angle between the orbital angular momentum and the $z$-axis while $\alpha$ is the angle between the $x$-axis and the projection of the orbital angular momentum onto the $x-y$ plane. For precessing binaries, as these angles vary in time, one must solve the precession equations to find their evolution. Notice that this source frame is the same as used in Ref. [34], and depicted in Fig. 2 of that paper.

We also find it useful to define basis vectors for the instantaneous orbital plane. These vectors have an implicit time dependence through the angles $(\iota, \alpha)$, and rotate about $\hat{\mathbf{L}}_{N}$ as it precesses. Here are their components in the $(\hat{\mathbf{x}}, \hat{\mathbf{y}}, \hat{\mathbf{z}})$ source basis:

$$
\begin{aligned}
& \hat{\mathbf{x}}_{L}=\frac{\mathbf{J}_{0} \times \hat{\mathbf{L}}_{\mathrm{N}}}{\left|\mathbf{J}_{0} \times \hat{\mathbf{L}}_{\mathrm{N}}\right|}=(-\sin \alpha, \cos \alpha, 0), \\
& \hat{\mathbf{y}}_{L}=\hat{\mathbf{L}}_{\mathrm{N}} \times \hat{\mathbf{x}}_{L}=(-\cos \iota \cos \alpha,-\cos \iota \sin \alpha, \sin \iota) .
\end{aligned}
$$

\footnotetext{
3 This can be seen explicitly from the relation linking $h_{\text {strain }}(t)$ to the complex polarization $h(t)$ introduced in Eq. 4.1): $h_{\text {strain }}=$ $\Re\left[h e^{2 i \bar{\Psi}}\left(e^{2 i \bar{\phi}} \cos ^{4}(\bar{\theta} / 2)+e^{-2 i \bar{\phi}} \sin ^{4}(\bar{\theta} / 2)\right)\right]$. The reader can easily check the equivalence with Eqs. (2.3), 2.4) and (2.5)

${ }^{4}$ Note that Ref. [34] chooses polarization vectors rotated by $\pi / 2$ relative to ours. This results in an overall sign difference from our polarizations, as can be seen by making the substitutions $\hat{\mathbf{P}} \rightarrow \hat{\mathbf{Q}}$ and $\hat{\mathbf{Q}} \rightarrow-\hat{\mathbf{P}}$, or by noting that GWs are spin-2 objects and flip sign under a $\pi / 2$ rotation. As mentioned, the polarization angle of this convention is then rotated by $\pi / 2$ relative to ours. This flips the sign of the antenna response functions as well, and so the same strain 2.3 is measured by either convention.
} 


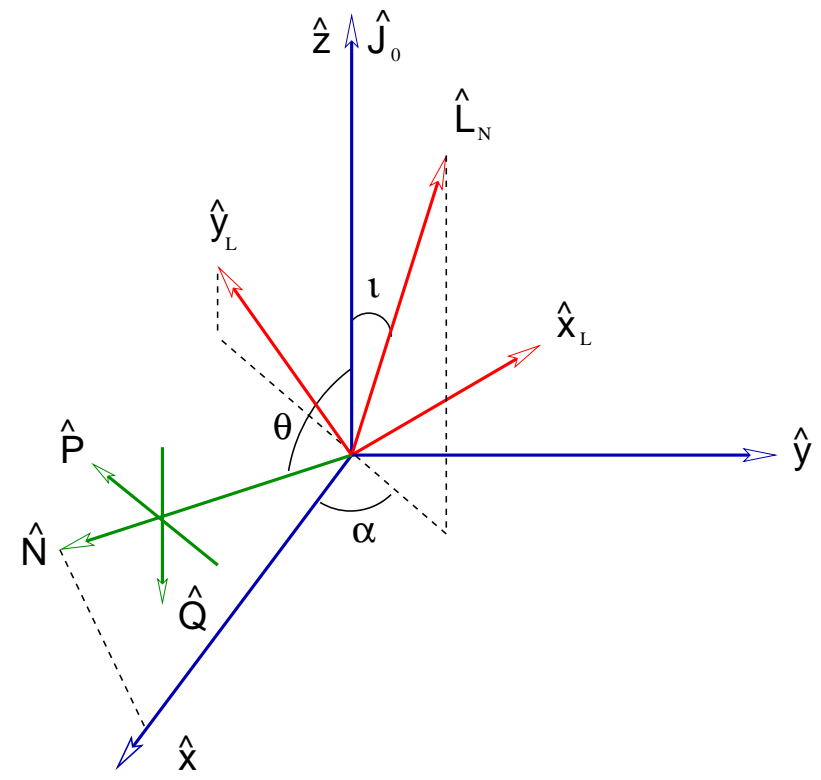

FIG. 1: We show (i) our source frame defined by the orthonormal basis $(\hat{\mathbf{x}}, \hat{\mathbf{y}}, \hat{\mathbf{z}})$, (ii) the instantaneous orbital plane which is described by the orthonormal basis $\left(\hat{\mathbf{x}}_{\mathrm{L}}, \hat{\mathbf{y}}_{\mathrm{L}}, \hat{\mathbf{L}}_{\mathrm{N}}\right)$, (iii) the polarization triad $(\hat{\mathbf{N}}, \hat{\mathbf{P}}, \hat{\mathbf{Q}})$, and (iv) the direction of the total angular momentum at initial time $\mathbf{J}_{0}$. Dashed lines show projections into the $x-y$ plane.

As an initial condition, we take the orbital separation vector $\hat{\mathbf{n}}$ to lie along $\hat{\mathbf{x}}_{L}$ at initial time, i.e., $\hat{\mathbf{n}}(t=0)=\hat{\mathbf{x}}_{L}(t=0)$. Then, we define the phase $\Phi(t)$ to be the cumulative angle between $\hat{\mathbf{x}}_{L}(t)$ and $\hat{\mathbf{n}}(t)$.

$$
\begin{aligned}
\hat{\mathbf{n}}(t) & =\hat{\mathbf{x}}_{L}(t) \cos \Phi(t)+\hat{\mathbf{y}}_{L}(t) \sin \Phi(t), \\
\hat{\boldsymbol{\lambda}}(t) & =-\hat{\mathbf{x}}_{L}(t) \sin \Phi(t)+\hat{\mathbf{y}}_{L}(t) \cos \Phi(t) .
\end{aligned}
$$

We thus see that the phase $\Phi(t)$ measures how $\hat{\mathbf{n}}$ has rotated relative to the vector $\hat{\mathbf{x}}_{L}$. However, for a precessing binary, $\hat{\mathbf{x}}_{L}$ is itself rotating about $\hat{\mathbf{L}}_{\mathrm{N}}$. This means that the total rotation of $\hat{\mathbf{n}}$ about $\hat{\mathbf{L}}_{\mathrm{N}}$ can be decomposed as a rotation of $\hat{\mathbf{n}}$ in the comoving basis parametrized by $\Phi(t)$ times a rotation parametrized by a precession phase due to the movement of the orbital plane itself. In the non-precessing case we have $\mathbf{J}_{0} \| \hat{\mathbf{L}}_{\mathrm{N}}$ and $\Phi(t)$ is expected to become the standard orbital phase whose time derivative is the orbital frequency. However, when $\mathbf{J}_{0} \| \hat{\mathbf{L}}_{\mathrm{N}}$ holds, we cannot define $\Phi=0$ for $\hat{\mathbf{n}}=\mathbf{J}_{0} \times \hat{\mathbf{L}}_{\mathrm{N}} /\left|\mathbf{J}_{0} \times \hat{\mathbf{L}}_{\mathrm{N}}\right|$, and we set $\Phi=0$ at the ascending node $\hat{\mathbf{N}} \times \hat{\mathbf{L}}_{\mathrm{N}}=\hat{\mathbf{P}}$, where the orbital separation crosses the plane of the sky from below. Now, $\Phi=0$ at the ascending node is achieved for $\hat{\mathbf{x}}_{L}=\hat{\mathbf{n}}$ and $\hat{\mathbf{P}}=\hat{\mathbf{n}}$, hence $\alpha=\pi$, so that the non-precessing regime is reached in the limit where $\iota=0$ and $\alpha=\pi$ for all time. This is applicable when the spins of the bodies are aligned or anti-aligned with the orbital angular momentum (or in the non-spinning limit). The waveforms are then greatly simplified.

We define the following mass parameters

$$
\begin{aligned}
M & =m_{1}+m_{2}, \\
\nu & =\frac{m_{1} m_{2}}{\left(m_{1}+m_{2}\right)^{2}}, \\
\mathcal{M} & =M \nu^{3 / 5}, \\
\delta & =\frac{m_{1}-m_{2}}{m_{1}+m_{2}}, \\
\nu & =\frac{1}{4}\left(1-\delta^{2}\right) .
\end{aligned}
$$

They are the total mass $M$, the symmetric mass ratio $\nu$, the fractional mass difference $\delta$, the chirp mass $\mathcal{M}$. The symmetric mass ratio is bounded according to $0<\nu \leq 1 / 4$ and the fractional mass difference satisfies $-1<\delta<1$.

The spin of a rotating compact body is of the order $\bar{S} \sim m l v_{\text {spin }}$ with $l \sim G m / c^{2}$. If the compact body is maximally rotating, then $v_{\text {spin }} \sim c$ and $S \sim \chi G \mathrm{~m}^{2} / c$. In words, from the PN point of view, the spin is formally of order 0.5PN. By contrast, if the compact body is slowly rotating, then $v_{\text {spin }} \ll c$, and the spin is formally of higher PN order, 
$S \sim \chi G m^{2} v_{\text {spin }} / c^{2} \sim 1 / c^{2}$. Throughout the paper, we use geometrical units where $G=c=1$. Henceforth, we shall work with the spin vectors normalized by the component masses as

$$
\chi_{n}=\frac{\mathbf{S}_{n}}{m_{n}^{2}}, \quad n=1,2,
$$

so that $\left|\chi_{n}\right| \leq 1$ for objects that obey the Kerr bound on rotational angular momentum. We also define symmetric and anti-symmetric spin combinations as in Ref. [35],

$$
\begin{aligned}
\chi_{s} & =\frac{1}{2}\left(\chi_{1}+\chi_{2}\right), \\
\chi_{a} & =\frac{1}{2}\left(\chi_{1}-\chi_{2}\right) .
\end{aligned}
$$

\section{READY-TO-USE GRAVITATIONAL-WAVE POLARIZATIONS FOR PRECESSING BINARIES ON CIRCULAR ORBITS THROUGH 1.5PN ORDER: SMALL INCLINATION ANGLES}

The expression of the strain tensor $h_{i j}$ for generic orbits through 1.5PN order was derived in Refs. [19, 35] and is given by Eq. (6.11) of Ref. [35]. In this section we compute ready-to-use polarizations in time domain through 1.5PN order within the adiabatic regime where the binary inspiral is modeled as a quasi-stationary sequence of orbits assumed to be nearly circular. By nearly circular, we essentially mean an orbit that would be exactly circular, with separation vector $\mathbf{r}_{0}$ of constant radius $r_{0}$, in the absence of spins and gravitational radiation. The perturbation of the separation $\delta r$ of such a motion is assumed to remain small with respect to $r_{0}$ on timescales on which the radiation-reaction effects can be neglected. This can only happen when the precession angles are at most of the same order of magnitude as the relative corrections induced by the spins in the dynamical quantities. Now, the evolution of $\delta r$ is governed by the radial part of the 1.5PN perturbation of the force per mass unit given in Eq. (2.1) of Ref. 34]. It turns out that this perturbation depends on the spin exclusively through the two projections $\left(\mathbf{S}_{n} \cdot \hat{\mathbf{L}}_{\mathrm{N}}\right)$ with $n=1,2$ which are almost constant apart from remainders that will contribute at higher orders in our weak precession hypothesis. In order to write the equation for $\delta r$, we project the relative acceleration a in the basis $\left\{\hat{\mathbf{n}}, \hat{\boldsymbol{\lambda}}, \hat{\boldsymbol{L}}_{\mathrm{N}}\right\}$. For the sake of convenience, we introduce an "orbital"-like frequency $\omega_{\text {orb }}$, defined as $\omega_{\text {orb }}=(\mathbf{v} \cdot \hat{\boldsymbol{\lambda}}) / r$. The closure relation yields the following decomposition for $\mathbf{v}$ and $\mathbf{a}$ :

$$
\begin{aligned}
& \mathbf{v}=\dot{r} \hat{\mathbf{n}}+\omega_{\text {orb }} r \hat{\boldsymbol{\lambda}} \\
& \mathbf{a}=\left(\ddot{r}-r \omega_{\text {orb }}^{2}\right) \hat{\mathbf{n}}+\left(r \dot{\omega}_{\text {orb }}+2 \dot{r} \omega_{\text {orb }}\right) \hat{\boldsymbol{\lambda}}-r \omega_{\text {orb }}\left(\hat{\boldsymbol{\lambda}} \cdot \frac{d \hat{\mathbf{L}}_{\mathrm{N}}}{d t}\right) \hat{\mathbf{L}}_{\mathrm{N}},
\end{aligned}
$$

with $\dot{r} \equiv d r / d t$. Splitting a into an unperturbed part $\mathbf{a}_{0}$ plus a perturbation $\delta \mathbf{a}$ and using the equations of motion, we find finally that $\delta r$ satisfies the equation $\delta \ddot{r}+\omega_{0}^{2} \delta r=$ const. where $\omega_{0}$ is the constant angular frequency of the background motion. A particular solution is given by a constant perturbation, $\delta r=$ const., whereas the homogeneous solution satisfies an harmonic oscillator equation independent of the spin.

By making the particular choice of a zero homogeneous solution, we can always eliminate the oscillations of $r$ that are not directly linked to the non-zero spins of the BHs. Based on these observations, we shall define precisely a nearly circular motion to be a perturbed circular motion whose homogeneous radial perturbation solution $(\delta r)_{\text {hom }}$ is zero, as it would be for an exactly circular motion. ${ }^{5}$ Assuming such a dynamics for our binary system implies that both $\delta r$ and $r=r_{0}+\delta r$ must be constant, provided we neglect higher order spin terms and radiative effects. We can generalize nearly circular motions to the case where spin precession angles are arbitrary in the absence of spin-spin interactions. This is achieved by introducing the concept of spherical motion defined as a motion having a constant separation $r$. It immediately follows from Eq. (3.1) that the full (conservative) acceleration is still of the form $-\omega_{\text {orb }}^{2} r \mathbf{n}$. Moreover, when radiation-reaction effects are neglected, the orbital frequency computed from the 1.5PN equations of motion keeps being almost constant [31, 34], even for precession angles that are no longer small. This can be seen [29] by noticing that the only possible non-constant terms in $\omega_{\text {orb }}$ at the $1.5 \mathrm{PN}$ order come from the leading spin contribution of the equations of motion, and thus, are of the form $\left(\mathbf{S}_{n} \cdot \hat{\mathbf{L}}_{\mathrm{N}}\right)$. Their time derivative reads

\footnotetext{
5 Though this type of motion can exist and is more general than the spin-aligned or anti-aligned case, it does not necessarily represent yet the most likely evolution to be observed.
} 
$\left(d \hat{\mathbf{L}}_{\mathrm{N}} / d t \cdot \mathbf{S}_{n}\right)+\left(\hat{\mathbf{L}}_{\mathrm{N}} \cdot d \mathbf{S}_{n} / d t\right)$. The first term is zero due to the precession equation $d \mathbf{S}_{n} / d t=\boldsymbol{\Omega}_{n} \times \mathbf{S}_{n}$, while the second term is a higher order correction quadratic in spins because of the approximate conservation of $\hat{\mathbf{L}}_{N}$. The treatment of the spin-spin dynamics is more delicate. A possible way to proceed consists in averaging the time dependent spin contributions in $\omega_{\text {orb }}$ over one orbital period 34,75$]$.

Introducing the invariant velocity,

$$
v \equiv\left(M \omega_{\text {orb }}\right)^{1 / 3}
$$

we reduce Eq. (6.11) of Ref. [35] to nearly circular orbits and expand it in powers of $v$ with the help of the relativistic extension of Kepler's law linking $\omega_{\text {orb }}$ and $M / r$ provided by Eq. (7.1) of Ref. [35]. Schematically, we obtain

$$
h_{i j}=\frac{2 M \nu v^{2}}{D_{L}}\left[\mathrm{Q}_{i j}+\mathrm{P}^{1 / 2} \mathrm{Q}_{i j} v+\left(\mathrm{P}^{1} \mathrm{Q}_{i j}+\mathrm{P}^{1} \mathrm{Q}_{i j}^{\mathrm{SO}}\right) v^{2}+\left(\mathrm{P}^{3 / 2} \mathrm{Q}_{i j}+\mathrm{P}^{3 / 2} \mathrm{Q}_{i j}^{\text {tail }}+\mathrm{P}^{3 / 2} \mathrm{Q}_{i j}^{\mathrm{SO}}\right) v^{3}\right]_{\mathrm{TT}},
$$

where SO indicates the spin-orbit terms; the tail integral $\mathrm{P}^{3 / 2} \mathrm{Q}_{i j}^{\text {tail }}$ given by Eq. (6.11e) of Ref. [35] reads

$$
\mathrm{P}^{3 / 2} \mathrm{Q}_{i j}^{\mathrm{tail}}=4\left[\pi\left(\hat{\lambda}^{i} \hat{\lambda}^{j}-\hat{n}^{i} \hat{n}^{j}\right)+12 \ln \left(\frac{v}{v_{0}}\right) \hat{\lambda}^{(i} \hat{n}^{j)}\right]_{\mathrm{TT}},
$$

$v_{0}$ being an arbitrary numerical constant reflecting the freedom in the choice of the radiative time origin. The symbol TT on the square bracket indicates the transverse trace-free projection in the plane orthogonal to the direction $\hat{\mathbf{N}}$ of the observer. We remind the reader that the non-spinning contributions to Eq. (3.3) are known through 3PN order 27, 28].

Apart from the spins, there are four vectors that appear in the expressions for the $\mathrm{P}^{n} \mathrm{Q}_{i j}$ 's in Eq. (3.3). In the source frame constructed in Sec. III they have the following $(x, y, z)$ components

$$
\begin{gathered}
\hat{\mathbf{n}}=(-\sin \alpha \cos \Phi-\cos \iota \cos \alpha \sin \Phi, \cos \alpha \cos \Phi-\cos \iota \sin \alpha \sin \Phi, \sin \iota \sin \Phi), \\
\hat{\boldsymbol{\lambda}}=(\sin \alpha \sin \Phi-\cos \iota \cos \alpha \cos \Phi,-\cos \alpha \sin \Phi-\cos \iota \sin \alpha \cos \Phi, \sin \iota \cos \Phi), \\
\hat{\mathbf{N}}=(\sin \theta, 0, \cos \theta), \\
\hat{\mathbf{L}}_{\mathrm{N}}=(\sin \iota \cos \alpha, \sin \iota \sin \alpha, \cos \iota),
\end{gathered}
$$

where $\Phi$ is the phase defined in Eq. (2.9) that measures how $\hat{\mathbf{n}}$ has rotated relative to the vector $\hat{\mathbf{x}}_{L}$. As $\hat{\mathbf{x}}_{L}$ is itself rotating about $\hat{\mathbf{L}}_{\mathrm{N}}$ for a precessing binary, the orbital frequency, or the total angular velocity of $\hat{\mathbf{n}}$ about $\hat{\mathbf{L}}_{\mathrm{N}}$, is the angular velocity of the motion of the binary within its instantaneous orbital plane, plus a precession velocity due to the movement of the orbital plane itself. To derive the relationship between the phase $\Phi(t)$ and the orbital phase (or carrier phase), we compute the derivative of $\hat{\mathbf{n}}(t)$ by means of Eqs. (2.7), (2.9), obtaining

$$
\frac{d \hat{\mathbf{n}}}{d t}=\left(\frac{d \Phi}{d t}+\cos \iota \frac{d \alpha}{d t}\right) \hat{\boldsymbol{\lambda}}+\left(\frac{d \iota}{d t} \sin \Phi-\sin \iota \cos \Phi \frac{d \alpha}{d t}\right) \hat{\mathbf{L}}_{\mathrm{N}}
$$

By imposing $\hat{\mathbf{L}}_{\mathrm{N}}=\hat{\mathbf{n}} \times \mathbf{v} /|\hat{\mathbf{n}} \times \mathbf{v}|=\hat{\mathbf{n}} \times d \hat{\mathbf{n}} / d t /|\hat{\mathbf{n}} \times d \hat{\mathbf{n}} / d t|$, and using Eq. (3.5) as well as Eq. (3.8), we find that the term proportional to $\hat{\mathbf{L}}_{\mathrm{N}}$ in Eq. (3.9) must be zero. Thus, we have $d \hat{\mathbf{n}} / d t=\dot{r} \mathbf{r} / r^{2}+\dot{\mathbf{r}} / r \equiv(\mathbf{v} \cdot \hat{\boldsymbol{\lambda}}) \hat{\boldsymbol{\lambda}}$, where $\mathbf{v} \cdot \hat{\boldsymbol{\lambda}}$ is the orbital frequency $\omega_{\text {orb }}$ defined before Eq. (3.1), which may be now interpreted as the angular velocity with which $\hat{\mathbf{n}}$ rotates about $\hat{\mathbf{L}}_{\mathrm{N}}$. Identification with Eq. (3.9) leads to the relation

$$
\omega_{\text {orb }}=\dot{\Phi}+\cos \iota \dot{\alpha}
$$

the phase $\Phi(t)$ being simply the integral

$$
\Phi(t)=\int_{0}^{t}\left[\omega_{\mathrm{orb}}\left(t^{\prime}\right)-\cos \iota\left(t^{\prime}\right) \dot{\alpha}\left(t^{\prime}\right)\right] d t^{\prime} .
$$

Due to the freedom in the choice of the time origin by the radiative observer, $h_{i j}$ depends on an undetermined time scale or, equivalently, on an arbitrary reference orbital frequency $\omega_{0}$. The constant $\omega_{0}$ is actually associated to the 
presence of gravitational-wave tails and appears solely in logarithms of the form $\ln \left(\omega_{\text {orb }} / \omega_{0}\right)$. Such contributions may be absorbed in the orbital phase by a redefinition of $\Phi$ into a shifted phase $\Psi$ [24]. Through $1.5 \mathrm{PN}$ order in the shift, we can pose $\Psi=\Phi-2 v^{3} \ln \left(\omega_{\text {orb }} / \omega_{0}\right)$. By plugging Eqs. (3.5)-(3.8) into Eq. (3.3), taking the combinations given in Eq. (2.1), and collecting terms by powers in $v$, we obtain the waveform polarizations

$$
h_{+, \times}=\frac{2 M \nu v^{2}}{D_{L}}\left[H_{+, \times}^{(0)}+H_{+, \times}^{(1 / 2)}+H_{+, \times}^{(1 / 2, \mathrm{SO})}+H_{+, \times}^{(1)}+H_{+, \times}^{(1, \mathrm{SO})}+H_{+, \times}^{(3 / 2)}+H_{+, \times}^{(3 / 2, \mathrm{SO})}\right] .
$$

The Newtonian, 0.5PN and 1PN order terms were already computed explicitly in Refs. 34, 35] [see in particular Eqs. (B2), (B3) of Ref. [34]], but as a series expansion of $M / r$ rather than $v$. Let us list for the reader convenience a few typographical errors we found there. In Eq. (4.9d) of Ref. 34], the factor of $(1 / 6)(149-6 \nu)$ has to be replaced with $(1 / 6)(149-36 \nu)$; in Eq. (B2c) $Q_{+}$must be changed to $-Q_{+}$; in Eq. (B3c) the right parenthesis is missing in the expression $\left(\cos ^{2} i \sin ^{2} \alpha+\cos ^{2} \alpha\right)$; at last, in Eq. (B3j) $c d$ should be read as $-c d$. In Ref. [35], Eq. (F14b) must be multiplied by $3 \nu$; in Eq. (F20) $-3 \nu$ has to be replaced with $+3 \nu$; there should be an overall minus sign in front of Eq. (F25c). If we re-expand Kidder's polarizations in $v$, and correct all the previous typos, we obtain complete agreement with both results through $1 \mathrm{PN}$ order. ${ }^{6}$

The lengthy expression for the GW polarizations can be reduced to a much more compact form by noticing (see also Sec. IVD in Ref. 34]) that in the limit $S \ll L$ the angle $\iota$ can be considered a 0.5PN order correction. This can be seen from

$$
\sin \iota=\frac{\left|\mathbf{J}_{0} \times \mathbf{L}\right|}{J_{0} L}
$$

if we neglect radiation reaction effects, i.e., we assume $\mathbf{J}_{0}=\mathbf{J}$, and use $\mathbf{J}=\mathbf{L}+\mathbf{S}_{1}+\mathbf{S}_{2}$ and $\mathbf{S}_{n}=\mathcal{O}(1 / c)$. We may then replace $\sin \iota$ and $\cos \iota$ in $h_{+, \times}$with their Taylor series expansions in $\iota$,

$$
\begin{aligned}
& \sin \iota=\iota-\frac{\iota^{3}}{6}+\mathcal{O}\left(\iota^{5}\right), \\
& \cos \iota=1-\frac{\iota^{2}}{2}+\mathcal{O}\left(\iota^{4}\right) .
\end{aligned}
$$

However, the assumption $S \ll L$ becomes less and less reliable for smaller mass ratio binaries. In fact, as a first approximation, we have $S_{n} / L=\left(m_{n} / M\right)^{2} \chi_{n} v / \nu$ with $v=\left(G M \omega_{\text {orb }} / c^{3}\right)^{1 / 3}$. Thus, even if $S_{n} \sim \mathcal{O}(1 / c), L$ can become comparable to $S_{n}$ when $\nu$ is sufficiently small. Moreover, we have assumed $\mathbf{J}_{0}=\mathbf{J}$ in Eq. (3.13), but the latter is not exact when radiation reaction is included, and it can be strongly violated in presence of transitional precession [76]. For these reasons, though we have decided to list in this section the GW polarizations expanded in $\iota$, we display in Appendix $\mathrm{A}$ the full expressions for generic inclination angles. For the $\iota$-expanded polarizations, we find

$$
\begin{aligned}
H_{+}^{(0)} & =-\left(c_{\theta}^{2}+1\right) \cos 2(\alpha+\Psi) \\
H_{+}^{(1 / 2)} & =v \delta s_{\theta}\left[\left(\frac{c_{\theta}^{2}}{8}+\frac{5}{8}\right) \cos (\alpha+\Psi)-\frac{9}{8}\left(c_{\theta}^{2}+1\right) \cos 3(\alpha+\Psi)\right] \\
H_{+}^{(1)} & =v^{2}\left[\left(-\frac{c_{\theta}{ }^{4}}{3}+\frac{3 c_{\theta}^{2}}{2}+\frac{19}{6}+\left(c_{\theta}^{4}+\frac{11 c_{\theta}{ }^{2}}{6}-\frac{19}{6}\right) \nu\right) \cos 2(\alpha+\Psi)\right. \\
& \left.+\frac{4}{3}\left(1-c_{\theta}{ }^{4}\right)(3 \nu-1) \cos 4(\alpha+\Psi)\right] \\
H_{+}^{(3 / 2)} & =v^{3}\left[\delta s_{\theta}\left(\frac{c_{\theta}{ }^{4}}{192}-\frac{5 c_{\theta}^{2}}{16}-\frac{19}{64}+\left(-\frac{c_{\theta}^{4}}{96}-\frac{c_{\theta}{ }^{2}}{8}+\frac{49}{96}\right) \nu\right) \cos (\alpha+\Psi)-2 \pi\left(c_{\theta}^{2}+1\right) \cos 2(\alpha+\Psi)\right.
\end{aligned}
$$

\footnotetext{
${ }^{6}$ It is also worth noting that Ref. [34] sets the origin of phase to be at a point referred to as the ascending node and defined to be the point where the orbital separation crosses the $x-y$ plane. This is in fact the same as our phase origin, $\hat{\mathbf{x}}_{L}=\mathbf{J}_{0} \times \hat{\mathbf{L}}_{\mathrm{N}} /\left|\mathbf{J}_{0} \times \hat{\mathbf{L}}_{\mathrm{N}}\right|$, but to reduce the possibility of confusion, we do not call this point the ascending node. We reserve this term to mean the point where the separation vector crosses the plane of the sky from below.
} 


$$
\begin{aligned}
& +\delta s_{\theta}\left(\left(-\frac{81 c_{\theta}^{4}}{128}+\frac{45 c_{\theta}^{2}}{16}+\frac{657}{128}\right)+\left(\frac{81 c_{\theta}^{4}}{64}+\frac{9 c_{\theta}^{2}}{8}-\frac{225}{64}\right) \nu\right) \cos 3(\alpha+\Psi) \\
& \left.+\delta s_{\theta} \frac{625}{384}\left(1-c_{\theta}^{4}\right)(2 \nu-1) \cos 5(\alpha+\Psi)\right] \\
& H_{+}^{(1 / 2, \mathrm{SO})}=-2 \iota c_{\theta} s_{\theta} \cos (\alpha+2 \Psi), \\
& H_{+}^{(1, \mathrm{SO})}=v^{2}\left[\left(c_{\theta}\left(\chi_{a}^{x}+\delta \chi_{s}^{x}\right)-s_{\theta}\left(\chi_{a}^{z}+\delta \chi_{s}^{z}\right)\right) \cos (\alpha+\Psi)-c_{\theta}\left(\chi_{a}^{y}+\delta \chi_{s}^{y}\right) \sin (\alpha+\Psi)\right] \\
& +v \iota \delta c_{\theta}\left[\frac{1}{4} s_{\theta}^{2} \cos \Psi-\left(\frac{c_{\theta}{ }^{2}}{8}+\frac{5}{8}\right) \cos (2 \alpha+\Psi)+\left(-\frac{9}{8}+\frac{27 c_{\theta}^{2}}{8}\right) \cos (2 \alpha+3 \Psi)\right] \\
& +\iota^{2}\left[-\frac{3}{2} s_{\theta}^{2} \cos 2 \Psi+\frac{1}{2}\left(c_{\theta}^{2}+1\right) \cos 2(\alpha+\Psi)\right] \\
& H_{+}^{(3 / 2, \mathrm{SO})}=v^{3}\left[s_{\theta} c_{\theta}\left(2 \delta \chi_{a}^{x}+(2-\nu) \chi_{s}^{x}\right)+\left(\frac{4}{3}\left(1+c_{\theta}{ }^{2}\right) \delta \chi_{a}^{z}+\frac{4}{3}\left(\left(1+c_{\theta}{ }^{2}\right)+\nu\left(1-5 c_{\theta}{ }^{2}\right)\right) \chi_{s}^{z}\right.\right. \\
& \left.\left.-s_{\theta} c_{\theta}\left(2 \delta \chi_{a}^{x}+(2+7 \nu) \chi_{s}^{x}\right)\right) \cos 2(\alpha+\Psi)-s_{\theta} c_{\theta}\left(2 \delta \chi_{a}^{y}+(2-\nu) \chi_{s}^{y}\right) \sin 2(\alpha+\Psi)\right] \\
& +v^{2} \iota s_{\theta}\left[c_{\theta}\left(-c_{\theta}^{2}+4+\left(3 c_{\theta}^{2}+\frac{2}{3}\right) \nu\right) \cos (\alpha+2 \Psi)+c_{\theta}\left(-\frac{c_{\theta}^{2}}{3}-1+\left(c_{\theta}^{2}+3\right) \nu\right) \cos (3 \alpha+2 \Psi)\right. \\
& \left.+c_{\theta}^{3}\left(\frac{16}{3}-16 \nu\right) \cos (3 \alpha+4 \Psi)-\left(\chi_{a}^{y}+\delta \chi_{s}^{y}\right) \sin \Psi\right]+v \iota^{2} s_{\theta} \delta\left[\left(-\frac{3 c_{\theta}^{2}}{16}+\frac{9}{16}\right) \cos (\alpha-\Psi)\right. \\
& -\left(\frac{11 c_{\theta}^{2}}{32}+\frac{23}{32}\right) \cos (\alpha+\Psi)+\frac{27}{32}\left(c_{\theta}^{2}+1\right) \cos 3(\alpha+\Psi)-\frac{1}{32}\left(c_{\theta}^{2}+1\right) \cos (3 \alpha+\Psi) \\
& \left.+\left(-\frac{45}{32}+\frac{135 c_{\theta}^{2}}{32}\right) \cos (\alpha+3 \Psi)\right]+\iota^{3} c_{\theta} s_{\theta}\left[\frac{1}{2} \cos (\alpha-2 \Psi)+\frac{5}{6} \cos (\alpha+2 \Psi)\right] \\
& H_{\times}^{(0)}=-2 c_{\theta} \sin 2(\alpha+\Psi), \\
& H_{\times}^{(1 / 2)}=v \delta c_{\theta} s_{\theta}\left[-\frac{9}{4} \sin 3(\alpha+\Psi)+\frac{3}{4} \sin (\alpha+\Psi)\right], \\
& H_{\times}^{(1)}=v^{2} c_{\theta}\left[\left(-\frac{4 c_{\theta}^{2}}{3}+\frac{17}{3}+\left(-\frac{13}{3}+4 c_{\theta}^{2}\right) \nu\right) \sin 2(\alpha+\Psi)+s_{\theta}^{2}\left(-\frac{8}{3}+8 \nu\right) \sin 4(\alpha+\Psi)\right] \\
& H_{\times}^{(3 / 2)}=v^{3} c_{\theta}\left[\delta s_{\theta}\left(\left(-\frac{21}{32}+\frac{5 c_{\theta}^{2}}{96}\right)+\left(-\frac{5 c_{\theta}^{2}}{48}+\frac{23}{48}\right) \nu\right) \sin (\alpha+\Psi)-4 \pi \sin 2(\alpha+\Psi)\right. \\
& +\delta s_{\theta}\left(\left(-\frac{135 c_{\theta}^{2}}{64}+\frac{603}{64}\right)+\left(-\frac{171}{32}+\frac{135 c_{\theta}^{2}}{32}\right) \nu\right) \sin 3(\alpha+\Psi) \\
& \left.+\delta s_{\theta}\left(\frac{625}{192}(2 \nu-1) s_{\theta}^{2}\right) \sin 5(\alpha+\Psi)\right] \\
& H_{\times}^{(1 / 2, \mathrm{SO})}=-2 \iota s_{\theta} \sin (\alpha+2 \Psi) \text {, } \\
& H_{\times}^{(1, \mathrm{SO})}=v^{2}\left[\left(\chi_{a}^{y}+\delta \chi_{s}^{y}\right) \cos (\alpha+\Psi)+c_{\theta}\left(c_{\theta}\left(\chi_{a}^{x}+\delta \chi_{s}^{x}\right)-s_{\theta}\left(\chi_{a}^{z}+\delta \chi_{s}^{z}\right)\right) \sin (\alpha+\Psi)\right] \\
& +\iota v \delta\left[s_{\theta}^{2} \sin \Psi-\left(\frac{c_{\theta}^{2}}{2}+\frac{1}{4}\right) \sin (2 \alpha+\Psi)+\left(-\frac{9}{4}+\frac{9 c_{\theta}^{2}}{2}\right) \sin (2 \alpha+3 \Psi)\right]+\iota^{2} c_{\theta} \sin 2(\alpha+\Psi)
\end{aligned}
$$




$$
\begin{aligned}
H_{\times}^{(3 / 2, \mathrm{SO})} & =v^{3}\left[s_{\theta}\left(2 \delta \chi_{a}^{y}+(2-\nu) \chi_{s}^{y}\right)(1+\cos 2(\alpha+\Psi))\right. \\
& \left.+\left(\frac{8}{3} c_{\theta} \delta \chi_{a}^{z}+c_{\theta}\left(\frac{8}{3}-\left(\frac{4}{3}+4 c_{\theta}^{2}\right) \nu\right) \chi_{s}^{z}-s_{\theta}\left(2 \delta \chi_{a}^{x}+\left(2+\left(3+4 c_{\theta}^{2}\right) \nu\right) \chi_{s}^{x}\right)\right) \sin 2(\alpha+\Psi)\right] \\
& +\iota v^{2} s_{\theta}\left[\left(c_{\theta}\left(\chi_{a}^{x}+\delta \chi_{s}^{x}\right)-s_{\theta}\left(\chi_{a}^{z}+\delta \chi_{s}^{z}\right)\right) \sin \Psi+\left(\left(-3 c_{\theta}^{2}+6\right)+\left(-\frac{16}{3}+9 c_{\theta}^{2}\right) \nu\right) \sin (\alpha+2 \Psi)\right. \\
& \left.+\left(-\left(c_{\theta}^{2}+\frac{1}{3}\right)+\left(3 c_{\theta}^{2}+1\right) \nu\right) \sin (3 \alpha+2 \Psi)+\left(\left(-\frac{8}{3}+8 c_{\theta}^{2}\right)+\left(-24 c_{\theta}^{2}+8\right) \nu\right) \sin (3 \alpha+4 \Psi)\right] \\
& +\iota^{2} v \delta c_{\theta} s_{\theta}\left[\frac{3}{8} \sin (\alpha-\Psi)-\frac{17}{16} \sin (\alpha+\Psi)+\frac{27}{16} \sin 3(\alpha+\Psi)-\frac{1}{16} \sin (3 \alpha+\Psi)+\frac{45}{16} \sin (\alpha+3 \Psi)\right] \\
& +\iota^{3}\left[\frac{1}{2} s_{\theta} \sin (\alpha-2 \Psi)+\frac{5}{6} s_{\theta} \sin (\alpha+2 \Psi)\right]
\end{aligned}
$$

where $s_{\theta}$ and $c_{\theta}$ are shorthand notations for $\sin \theta$ and $\cos \theta$ respectively. In Sec. IV (see Fig. 2), we shall discuss typical variations of the inclination angle $\iota$ depending on spin orientations and binary mass ratios. Note that whereas the terms of $H_{+, \times}^{(1, \mathrm{SO})}$ linear in $\chi_{n}$ depend on the first harmonic of the orbital frequency, those of $H_{+, \times}^{(3 / 2, \mathrm{SO})}$ depend on its zeroth and second harmonic, and so do the terms of $H_{+, \times}^{(2, \mathrm{SS})}$ quadratic in the spin components, although we do not use them here. We include these 2PN SS polarization corrections when constructing frequency-domain waveforms for binaries having their spins aligned or anti-aligned with the orbital angular momentum in Sec. VI] The harmonic dependence of the polarization corrections produced by the spins can be understood from the explicit expression for $h_{i j}$ shown in Eqs. (4.9c) and (4.9d) of Ref. 34] or Eqs. (F15a)-(F15c) of Ref. 35]. The 1PN SO contributions are proportional to the components of the orbital separation vector, $\hat{\mathbf{n}}$, which are themselves proportional to $\sin \Phi$ and $\cos \Phi$, so that $H_{+, \times}^{(1, \mathrm{SO})}$ depend on the first harmonic of the orbital phase. Next, the 1.5PN SO and 2PN SS contributions are proportional to products of the orbital separation or instantaneous velocity unit vectors, $\hat{\mathbf{n}}$ or $\hat{\boldsymbol{\lambda}}$, and to products of $\sin \Phi$ or $\cos \Phi$. These can be re-expressed in terms of $\sin 2 \Phi, \cos 2 \Phi$ or constant quantities independent of $\Phi$, so that $H_{+, \times}^{(3 / 2, \mathrm{SO})}$ and $H_{+, \times}^{(2, \mathrm{SS})}$ depend on the zeroth and second harmonics of the orbital phase. Because the expressions for $h_{i j}$ in Refs. [34, 35] are expanded in $(M / r)$, while we use an expansion in $v=\left(M \omega_{\text {orb }}\right)^{1 / 3}$, one has to convert from one expansion to the other by using Eqs. (7.1) and (F20) of Ref. [35]. In doing so, the $v$-expansion gains additional 1.5PN SO and 2PN SS corrections proportional to the Newtonian order term depending on the second harmonic of the orbital phase. The 1PN SO term is left unchanged.

Ready-to-use time-domain GW polarizations for spinning, precessing binaries through 1.5PN order in phase and amplitude can be obtained by solving numerically the following equations: (i) The spin precession equations [29, 30]

$$
\begin{aligned}
\frac{d \mathbf{S}_{1}}{d t} & =\boldsymbol{\Omega}_{1} \times \mathbf{S}_{1}, \\
\frac{d \mathbf{S}_{2}}{d t} & =\boldsymbol{\Omega}_{2} \times \mathbf{S}_{2},
\end{aligned}
$$

where at $1.5 \mathrm{PN}$ order

$$
\boldsymbol{\Omega}_{1,2}=\omega_{\mathrm{orb}}^{5 / 3}\left(\frac{3}{4}+\frac{\nu}{2} \mp \frac{3}{4} \delta\right) \hat{\mathbf{L}}_{\mathrm{N}}
$$

(ii) The evolution equation for the Newtonian angular momentum

$$
\dot{\hat{L}}_{\mathrm{N}}=-\frac{v}{\nu}\left(\dot{\mathbf{S}}_{1}+\dot{\mathbf{S}}_{2}\right)
$$

(iii) The equation for the orbital frequency

$$
\frac{\dot{\omega}_{\text {orb }}}{\omega_{\text {orb }}^{2}}=\frac{96}{5} \nu v^{5}\left\{1-\left(\frac{743}{336}+\frac{11}{4} \nu\right) v^{2}+\left[\left(\frac{19}{3} \nu-\frac{113}{12}\right) \chi_{s} \cdot \hat{\mathbf{L}}_{\mathrm{N}}-\frac{113}{12} \delta \boldsymbol{\chi}_{a} \cdot \hat{\mathbf{L}}_{\mathrm{N}}\right] v^{3}+4 \pi v^{3}\right\} .
$$

Integrating $\omega_{\text {orb }}$ yields the orbital phase $\Phi_{\text {orb }}(t) \equiv \int_{0}^{t} \omega_{\text {orb }}\left(t^{\prime}\right) d t^{\prime}$. The GW polarizations (3.12) through 1.5PN order in phase and amplitude are computed by solving numerically Eqs. (3.11), (3.18), (3.20), and (3.21). In order to compute 
the GW polarizations (3.12) through 1.5PN order in amplitude, but at the highest available $\mathrm{PN}$ order in phase, one should replace Eqs. (3.18), (3.20) with Eq. (7.5) in Ref. [30] and Eq. (32) in Ref. [60], respectively.

\section{GRAVITATIONAL-WAVE MODES FOR PRECESSING BINARIES ON NEARLY CIRCULAR ORBITS THROUGH 1.5PN ORDER: SMALL INCLINATION ANGLES}

Due to the spin-2 nature of GWs, it is convenient to decompose the waveform components in the dyad $\{(\mathbf{P}+$ $i \mathbf{Q}) / \sqrt{2},(\mathbf{P}-i \mathbf{Q}) / \sqrt{2}\}$ with respect to an orthonormal basis of spin \pm 2 functions that are defined on the 2 -sphere and belong to an irreducible representation of $\mathrm{SO}(3)$. Most commonly, the complex polarization

$$
h=h_{j k} \frac{P^{j}-i Q^{j}}{\sqrt{2}} \frac{P^{k}-i Q^{k}}{\sqrt{2}}=h_{+}-i h_{\times},
$$

is expanded into the set of spin-weighted -2 spherical harmonics. Like the standard spherical harmonics, these functions of the two angles of spherical coordinates are labeled by a pair of integers, say $(\ell, m)$, with $\ell \geq 2$ and $m \leq|\ell|$. The spin-weighted $-s$ spherical harmonics associated to any such pair are given by ${ }^{7}$ [77]

$$
{ }_{-s} Y^{\ell m}(\theta, \phi)=(-1)^{s} \sqrt{\frac{2 \ell+1}{4 \pi}} d_{s m}^{\ell}(\theta) e^{i m \phi},
$$

with

$$
d_{s m}^{\ell}(\theta)=\sum_{k=\max (0, m-s)}^{\min (\ell+m, \ell-s)} \frac{(-1)^{k}}{k !} \frac{\sqrt{(\ell+m) !(\ell-m) !(\ell+s) !(\ell-s) !}}{(k-m+s) !(\ell+m-k) !(\ell-k-s) !}\left(\cos \frac{\theta}{2}\right)^{2 \ell+m-2 k-s}\left(\sin \frac{\theta}{2}\right)^{2 k-m+s}
$$

and the orthogonality relation holds

$$
\int d \Omega_{-s} Y^{\ell m}(\theta, \phi)_{-s} Y^{\ell^{\prime} m^{\prime} *}(\theta, \phi)=\delta^{\ell \ell^{\prime}} \delta^{m m^{\prime}}
$$

where $d \Omega=\sin \theta d \theta d \phi$ denotes the element of solid angle and $\delta^{\ell \ell^{\prime}}$ is the Kronecker symbol. The integration is performed over the unit sphere, so that $0 \leq \theta \leq \pi$ and $0 \leq \phi \leq 2 \pi$. The mode expansion of the complex polarization (4.1) is then of the form

$$
h(\theta, \phi)=\sum_{\ell=2}^{+\infty} \sum_{m=-\ell}^{\ell} h_{\ell m-2} Y^{\ell m}(\theta, \phi) .
$$

The GW modes $h_{\ell m}$ are extracted using the orthogonality property (4.4) by means of the surface integral

$$
h_{\ell m}=\int d \Omega h(\theta, \phi)_{-2} Y^{\ell m *}(\theta, \phi),
$$

where the star on the spin-weighted -2 harmonic indicates the complex conjugation. Therefore, the calculation of $h_{\ell m}$ requires the knowledge of the polarizations $h_{+}$and $h_{\times}$for an arbitrary value of the azimuthal angle $\phi$ of the direction $\hat{\mathbf{N}}$. In Sec. III we have computed $h_{+}$and $h_{\times}$only for $\phi=0$; however, a specific choice of the $x$-axis orientation cannot be responsible for any information loss. Thus, we must be able to recover $h(\theta, \phi)$ from the expression of $h(\theta, 0)$ alone.

The quantity $h$ at a given point depends on a number of parameters, such as $\iota$ or the spin variables, and can actually be regarded as a function of $\theta, \phi$ as well as a function of the whole set of parameters that possess a geometrical character. More precisely, we may write $h(\theta, \phi)=\bar{h}\left(\theta, \phi, \iota, \alpha, \Phi, \chi_{n}^{x}, \chi_{n}^{y}, \chi_{n}^{z}\right)$. Let us now introduce the projection basis $\left(\hat{\mathbf{x}}^{\prime}, \hat{\mathbf{y}}^{\prime}, \hat{\mathbf{z}}^{\prime}=\hat{\mathbf{z}}\right)$ obtained by applying a rotation of angle $\phi$ about the $z$-axis on the vectors of the original basis $(\hat{\mathbf{x}}, \hat{\mathbf{y}}, \hat{\mathbf{z}})$. Let us also associate to each variable of $\bar{h}$ a primed counterpart, which is defined in the same way as the unprimed variable but refers to the new basis rather than the original one. For instance, $\alpha^{\prime}$ denotes the azimuthal

\footnotetext{
7 Our definition of ${ }_{-s} Y^{\ell m}(\theta, \phi)$ differs from that of Ref. [7] by a factor $(-1)^{m}$ so that ${ }_{0} Y^{\ell m}(\theta, \phi)$ coincides with the most broadly used definition of $Y^{\ell m}(\theta, \phi)$; for the d-matrix $d_{m^{\prime} m}^{\ell}(\theta)$, we adopt the same convention as Landau-Lifchitz [78].
} 
angle of the orbital angular momentum measured from the fixed vector $\hat{\mathbf{x}}^{\prime}$ instead of $\hat{\mathbf{x}}$. In particular, we have $\theta^{\prime}=\theta$, $\phi^{\prime}=0, \iota^{\prime}=\iota, \alpha^{\prime}=\alpha-\phi$. The phase $\Phi$, defined as the angle $\left(\hat{\mathbf{L}}_{\mathrm{N}} \times \hat{\mathbf{z}}, \hat{\mathbf{n}}\right)=\left(\hat{\mathbf{L}}_{\mathrm{N}} \times \hat{\mathbf{z}}^{\prime}, \hat{\mathbf{n}}\right)$, is not affected by the transformation: $\Phi^{\prime}=\Phi$. The $x^{\prime}$ and $y^{\prime}$ spin components can be obtained from the 2-dimensional formula for a passive rotation of angle $\phi$, that is

$$
\begin{aligned}
& \chi_{n}^{\prime x}=\chi_{n}^{x} \cos \phi+\chi_{n}^{y} \sin \phi, \\
& \chi_{n}^{\prime y}=-\chi_{n}^{x} \sin \phi+\chi_{n}^{y} \cos \phi,
\end{aligned}
$$

while the third component is left unchanged. With our conventions, the polarization vectors in the new basis remain equal to $\mathbf{P}$ and $\mathbf{Q}$ respectively. Therefore, the complex polarization is identical to that of the old frame. Moreover, by construction of the primed variables, the functional dependence of $h$ is the same as before, meaning that $h=$ $\bar{h}\left(\theta, \phi, \iota, \alpha, \Phi, \chi_{n}^{x}, \chi_{n}^{y}, \chi_{n}^{z}\right)=\bar{h}\left(\theta^{\prime}, \phi^{\prime}, \iota^{\prime}, \alpha^{\prime}, \Phi^{\prime}, \chi_{n}^{\prime x}, \chi_{n}^{\prime y}, \chi_{n}^{\prime z}\right)$. Hence the important relation

$$
h \equiv \bar{h}\left(\theta, \phi, \iota, \alpha, \Phi, \chi_{n}^{x}, \chi_{n}^{y}, \chi_{n}^{z}\right)=\bar{h}\left(\theta, 0, \iota, \alpha-\phi, \Phi, \cos \phi \chi_{n}^{x}+\sin \phi \chi_{n}^{y},-\sin \phi \chi_{n}^{x}+\cos \phi \chi_{n}^{y}, \chi_{n}^{z}\right),
$$

where the function $\bar{h}\left(\theta, 0, \iota, \alpha, \chi_{n}^{x}, \chi_{n}^{y}, \chi_{n}^{z}\right)$ is given by Eqs. (3.16), (3.17) for the $\iota$-expanded expressions or by Eqs. (A2), (A3) for the full ones.

At $1.5 \mathrm{PN}$ order, the GW polarizations decompose into a sum of 3 terms, $h_{0}(\theta, \iota, \alpha, \Phi)+\sum_{n=1,2} \chi_{n} . \mathbf{h}_{n}(\theta, \iota, \alpha, \Phi)$, which shows that $h$ may be written as

$$
h_{0}(\theta, \iota, \alpha-\phi, \Phi)+\sum_{n=1,2}\left[e^{i \phi} \zeta_{n}^{*} k_{n}(\theta, \iota, \alpha-\phi, \Phi)+e^{-i \phi} \zeta_{n} k_{n}^{*}(\theta, \iota, \alpha-\phi, \Phi)+\chi_{n}^{z} h_{n}^{z}(\theta, \iota, \alpha-\phi, \Phi)\right]
$$

with $\zeta_{n}=\left(\chi_{n}^{x}+i \chi_{n}^{y}\right) / \sqrt{2}$ and $k_{n}=\left(h_{n}^{x}+i h_{n}^{y}\right) / \sqrt{2}$. Each mode $h_{\ell m}$ splits accordingly into 7 contributions: the spinfree term and 6 terms proportional to each of the spin variable components. These contributions are parametrized by a vector weight $m^{\prime}=-1,0,1$, as well as the body label $n=1,2$ of the spins; $n=0$ refers to quantities entering the spin-free part of $h$ for which we also set $m^{\prime}=0$. As a result, for precessing binaries, the integral to compute $h_{\ell m}$ takes the form:

$$
h_{\ell m}=\sum_{m^{\prime}=-1}^{1} \sum_{n=1}^{2} X_{m^{\prime}, n} \int d \Omega K_{m^{\prime}, n}(\theta, \iota, \alpha-\phi, \Phi) e^{i\left(-m^{\prime}-m\right) \phi}{ }_{-2} Y^{\ell m *}(\theta, 0),
$$

where $X_{0,0}=1, X_{0, n^{\prime}}=\chi_{n^{\prime}}^{z}\left(\right.$ for $\left.n^{\prime}=1,2\right), X_{-1, n^{\prime}}=\zeta_{n^{\prime}}^{*}, X_{1, n^{\prime}}=\zeta_{n^{\prime}}, K_{0,0}=h_{0}, K_{0, n^{\prime}}=h_{n^{\prime}}^{z}, K_{-1, n^{\prime}}=k_{n^{\prime}}$ and $K_{1, n^{\prime}}=k_{n^{\prime}}^{*}$. By means of the change of variable $\phi \rightarrow \phi+\alpha$, we are able to factor out a complex exponential $e^{-i\left(m+m^{\prime}\right) \alpha}$ which contain all the dependence in $\alpha$. Let us now focus henceforth on the case where the waveform has been expanded in powers of $\iota$. As we shall explicitly see below, [see Eq. (4.17)], the $h_{\ell m}$ 's are then made of: (i) a spin-free piece proportional to $e^{-i m \alpha}$, (ii) two spin pieces proportional to $e^{-i(m-1) \alpha}$ and to $\zeta_{1}^{*}$ or $\zeta_{2}^{*}$ respectively, (iii) two spin pieces proportional to $e^{-i(m+1) \alpha}$ and to $\zeta_{1}$ or $\zeta_{2}$ respectively, (iv) two spin pieces proportional to $e^{-i m \alpha}$ and to $\chi_{1}^{z}$ or $\chi_{2}^{z}$ respectively. In contrast to what happens in the non-spinning case, $h_{\ell m}$ is not in general proportional to $e^{-i m \Phi}$ except for the terms that are free of $\iota$, since both $\hat{\mathbf{n}}$ and $\hat{\boldsymbol{\lambda}}$ reduce to trigonometric functions of $\Phi+\alpha$ as $\iota \rightarrow 0$. The contributions to the polarization modes that are linear in $\iota$ involve couplings of the type $e^{-i\left(m+m^{\prime}\right)(\Phi+\alpha)} \cos \Phi$ or $e^{-i\left(m+m^{\prime}\right)(\Phi+\alpha)} \sin \Phi$ because the terms of first order in $\iota$ entering $\hat{\mathbf{n}}$ and $\hat{\boldsymbol{\lambda}}$ can only be linear combinations of $\iota$ cos $\Phi$ or $\iota \sin \Phi$ (or equivalently $\left.\iota e^{ \pm i \Phi}\right)$. Couplings like $e^{-i\left(m+m^{\prime}\right)(\Phi+\alpha)} \cos ^{a} \Phi \sin ^{b} \Phi$, with $a, b \in \mathbb{N}$, arise at higher orders making the dependence in $\Phi$ more complicated. A close inspection to the results below [see Eqs. 4.17a - 4.17r with $\Psi \rightarrow \Phi]$ confirm these expectations. Beware that our mode normalization is tuned to factor out the exponential factors $e^{-i m \alpha}$.

The structure of the modes is much more complicated for precessing binaries than for non-precessing binaries. When the orbital angular momentum is aligned with the total angular momentum $(\iota=0, \alpha=\pi)$, note that a rotation by $\phi$ about the z-axis produces an offset in the orbital phase angle, so that

$$
\bar{h}(\theta, \phi, \Phi)=\bar{h}(\theta, 0, \Phi-\phi)
$$

This ensures that only terms proportional to $e^{-i m \Phi}$ contribute to the integral over $\phi$ to compute $h_{\ell m}$. In the precessing case, a rotation by $\phi$ produces an offset in the $\alpha$ angle, and so terms with different powers of $e^{-i \Phi}$ can contribute to the same $h_{\ell m}$ mode. As we will see below, these terms with different powers of $e^{-i \Phi}$ interfere to produce rather complicated modulations to the modes on the orbital time scale. Since the precessional motion is typically much slower than the orbital motion (several orbital cycles are completed in any precessional cycle for the systems we consider), it may be surprising that the relatively slow precessional motion can produce such rapid oscillations in the 
modes. This is simply a breakdown of the nice structure (i.e. that $h_{\ell m} \propto e^{-i m \Phi}$ ) of the $h_{\ell m}$ modes in the precessing case. Note however, that what is actually observed are the gravitational wave polarizations. In the polarizations, precessional effects are indeed on a slower time scale than the orbital motion. They modulate the "envelope" of the waveform, rather than create orbital timescale interference.

A useful property of $\bar{h}$ coming from the arbitrariness of the body labeling is that it must be invariant in the exchange of particles 1 and 2: $m_{1} \leftrightarrow m_{2}, \chi_{1} \leftrightarrow \chi_{2}, \mathbf{n} \rightarrow-\mathbf{n}, \mathbf{v} \rightarrow-\mathbf{v}$. Under this transformation, the direction of the angular momentum $\hat{\mathbf{L}}$ remains invariant hence $\boldsymbol{\lambda} \rightarrow-\boldsymbol{\lambda}$. The orbital frequency $\omega_{\text {orb }}=(\mathbf{v} \cdot \boldsymbol{\lambda})$ is unchanged as well as the direction of the total angular momentum, due to its structure and parity. Therefore, the phase $\Phi$ becomes $\left(\hat{\mathbf{x}}_{L},-\hat{\mathbf{n}}\right)=\Phi+\pi$ whereas the angles $\alpha$ and $\iota$ are unaffected. This yields the relation

$$
\bar{h}\left(\theta, \phi, \iota, \alpha, \Phi, \chi_{1}^{x}, \chi_{2}^{x}, \chi_{1}^{y}, \chi_{2}^{y}, \chi_{1}^{z}, \chi_{2}^{z}\right)=\left[\bar{h}\left(\theta, \phi, \iota, \alpha, \Phi+\pi, \chi_{2}^{x}, \chi_{1}^{x}, \chi_{2}^{y}, \chi_{1}^{y}, \chi_{2}^{z}, \chi_{1}^{z}\right)\right]_{\delta \rightarrow-\delta} .
$$

The previous identity may be supplemented by another one which originates from the classical parity invariance of physics: for any given time instant $t$, the waveform resulting from the stress-energy tensor parametrized by the worldlines $\mathbf{x}_{n}$ and the spins $\chi_{n}$ must have the same value at point $\mathbf{x}$ as the waveform resulting from $-\mathbf{x}_{n}$ and $+\chi_{n}$ at point $-\mathbf{x}$. Taking into account the transformation of the polarization vectors under parity, this means for the function $\bar{h}$ :

$$
\bar{h}\left(\theta, \phi, \iota, \alpha, \Phi, \chi_{n}^{x}, \chi_{n}^{y}, \chi_{n}^{z}\right)=\bar{h}^{*}\left(\pi-\theta, \phi+\pi, \iota, \alpha, \Phi+\pi, \chi_{n}^{x}, \chi_{n}^{y}, \chi_{n}^{z}\right) .
$$

The above formula allows us to express the modes $h_{\ell m}$ in terms of the modes $h_{\ell-m}$ by performing the change of variable $\theta \rightarrow \pi-\theta$ and $\phi \rightarrow \phi+\pi$ in Eq. (4.6). The first factor of the integrand can be then rewritten as $\bar{h}^{*}(\theta, \phi, \iota, \alpha, \Phi+$ $\left.\pi, \chi_{n}^{x}, \chi_{n}^{y}, \chi_{n}^{z}\right)$ making use of Eq. (4.14). The second factor ${ }_{-2} Y^{\ell m *}(\pi-\theta, \phi+\pi)$ may be transformed by means of two important symmetry properties of the spin-weighted spherical harmonics: ${ }_{2} Y^{\ell m}(\pi-\theta, \phi+\pi)=(-1)^{\ell}+2 Y^{\ell m}(\theta, \phi)$ and ${ }_{+2} Y^{\ell m}(\theta, \phi)=(-1)^{m}-2 Y^{\ell-m *}(\theta, \phi)$, which leads to the new expression $(-1)^{m+\ell}{ }_{2} Y^{\ell-m}(\theta, \phi)$ for this factor. As a consequence, the link between $h_{\ell m}(\Phi) \equiv \int d \Omega \bar{h}\left(\theta, \phi, \iota, \alpha, \Phi, \chi_{n}^{x}, \chi_{n}^{y}, \chi_{n}^{z}\right)_{-2} Y^{\ell m *}(\theta, \phi)$ and $h_{\ell-m}(\Phi)$ is given by

$$
h_{\ell m}(\Phi)=(-1)^{m+\ell} \int d \Omega \bar{h}^{*}\left(\theta, \phi, \iota, \alpha, \Phi+\pi, \chi_{n}^{x}, \chi_{n}^{y}, \chi_{n}^{z}\right)_{-2} Y^{\ell-m}(\theta, \phi)=(-1)^{\ell+m} h_{\ell-m}^{*}(\Phi+\pi)
$$

The explicit expressions for the modes $h_{\ell m}$ are obtained by inserting Eq. (4.9) into the surface integral (4.4). We normalize them in such a way that the leading order mode starts with coefficient 1 . Posing

$$
h_{\ell m}=-\frac{\left(2 M \nu v^{2}\right)}{D_{L}} \sqrt{\frac{16 \pi}{5}} e^{-i m(\Psi+\alpha)} \hat{h}_{\ell m}
$$

and expanding in the inclination angle $\iota$, we arrive $a t^{8}$

$$
\begin{aligned}
\hat{h}_{22} & =1+\frac{1}{3} e^{i \Psi} \delta v \iota+v^{2}\left\{\frac{1}{42}(-107+55 \nu)-\frac{1}{2} e^{i(\alpha+\Psi)}\left[\chi_{a}^{x}-i \chi_{a}^{y}+\delta\left(\chi_{s}^{x}-i \chi_{s}^{y}\right)\right]\right\}-\frac{\iota^{2}}{2}+v^{3}\left[2 \pi-\frac{4 \delta \chi_{a}^{z}}{3}\right. \\
& \left.+\frac{4}{3}(-1+\nu) \chi_{s}^{z}\right]+\mathcal{O}\left(\frac{1}{c^{4}}\right) \\
\hat{h}_{21} & =-\frac{\delta v}{3}+e^{-i \Psi} \iota+\frac{v^{2}}{2}\left(\chi_{a}^{z}+\delta \chi_{s}^{z}\right)+\delta \iota^{2} v\left(\frac{5}{12}-\frac{1}{4} e^{2 i \Psi}\right)+v^{3}\left\{-e^{i(\alpha+\Psi)}\left[\delta\left(\chi_{a}^{x}-i \chi_{a}^{y}\right)+\left(1-\frac{\nu}{2}\right)\left(\chi_{s}^{x}-i \chi_{s}^{y}\right)\right]\right. \\
& \left.+e^{-i(\alpha+\Psi)}\left[\delta\left(\chi_{a}^{x}+i \chi_{a}^{y}\right)+\left(1+\frac{5}{6} \nu\right)\left(\chi_{s}^{x}+i \chi_{s}^{y}\right)\right]+\frac{\delta}{84}(17-20 \nu)\right\}+v^{2} \iota\left\{\frac{e^{-i \Psi}}{42}(-107+55 \nu)\right. \\
& \left.+\frac{1}{4}\left(\chi_{a}^{x}-i \chi_{a}^{y}+\delta\left(\chi_{s}^{x}-i \chi_{s}^{y}\right)\right) e^{i \alpha}\left(-1+e^{2 i \Psi}\right)\right\}+\frac{\iota^{3}}{4}\left(-\frac{5}{3} e^{-i \Psi}-e^{3 i \Psi}\right)+\mathcal{O}\left(\frac{1}{c^{4}}\right) \\
\hat{h}_{20} & =\frac{1}{2} \sqrt{\frac{3}{2}}\left\{\frac{v^{2}}{3}\left[-e^{i(\alpha+\Psi)}\left(\chi_{a}^{x}-i \chi_{a}^{y}+\delta\left(\chi_{s}^{x}-i \chi_{s}^{y}\right)\right)+e^{-i(\alpha+\Psi)}\left(\chi_{a}^{x}+i \chi_{a}^{y}+\delta\left(\chi_{s}^{x}+i \chi_{s}^{y}\right)\right)\right]\right. \\
& \left.+\frac{4 i}{3} v \iota \delta \sin \Psi+2 \iota^{2} \cos 2 \Psi-\frac{4 i}{3} v^{2} \iota \sin \Psi\left(\chi_{a}^{z}+\delta \chi_{s}^{z}\right)\right\}+\mathcal{O}\left(\frac{1}{c^{4}}\right)
\end{aligned}
$$

\footnotetext{
8 In the case of spins aligned or anti-aligned with the Newtonian angular momentum, the modes $(2,2),(2,1)$ and $(3,2)$ were also computed in Ref. [71]. We fully agree with their results.
} 


$$
\begin{aligned}
& \hat{h}_{33}=-\frac{3}{4} \sqrt{\frac{15}{14}}\left\{\delta v+v^{3}\left[2 \delta(-2+\nu)+\frac{16}{9} e^{i(\alpha+\Psi)} \nu\left(\chi_{s}^{x}-i \chi_{s}^{y}\right)\right]-\frac{4}{9} e^{i \Psi} \iota v^{2}(-1+3 \nu)\right. \\
& \left.+\frac{\delta v \iota^{2}}{4}\left(-3+\frac{e^{2 i \Psi}}{9}\right)\right\}+\mathcal{O}\left(\frac{1}{c^{4}}\right) \\
& \hat{h}_{32}=-\frac{9}{8} \sqrt{\frac{5}{7}}\left[\frac{8}{27} v^{2}(-1+3 \nu)+\delta v \iota\left(e^{-i \Psi}-\frac{e^{i \Psi}}{27}\right)-\frac{32}{27} v^{3} \nu \chi_{s}^{z}\right]+\mathcal{O}\left(\frac{1}{c^{4}}\right), \\
& \hat{h}_{31}=-\frac{1}{12 \sqrt{14}}\left\{\delta v+v^{3}\left[-\frac{2}{3} \delta(4+\nu)-16 e^{-i(\alpha+\Psi)} \nu\left(\chi_{s}^{x}+i \chi_{s}^{y}\right)\right]+20 v^{2} \iota(-1+3 \nu) e^{-i \Psi}\right. \\
& \left.+\frac{\delta v \iota^{2}}{2}\left(-\frac{11}{2}+\frac{135}{2} e^{-2 i \Psi}-3 e^{2 i \Psi}\right)\right\}+\mathcal{O}\left(\frac{1}{c^{4}}\right), \\
& \hat{h}_{30}=-\frac{1}{2 \sqrt{42}} \delta v \iota \cos \Psi+\mathcal{O}\left(\frac{1}{c^{4}}\right) \text {, } \\
& \hat{h}_{44}=\frac{8}{9} \sqrt{\frac{5}{7}} v^{2}(1-3 \nu)+\mathcal{O}\left(\frac{1}{c^{4}}\right) \text {, } \\
& \hat{h}_{43}=\frac{8}{9} \sqrt{\frac{10}{7}}\left[\frac{81}{320} v^{3} \delta(-1+2 \nu)+v^{2} \iota(1-3 \nu)\left(e^{-i \Psi}-\frac{e^{i \Psi}}{16}\right)\right]+\mathcal{O}\left(\frac{1}{c^{4}}\right), \\
& \hat{h}_{42}=\frac{\sqrt{5}}{63} v^{2}(1-3 \nu)+\mathcal{O}\left(\frac{1}{c^{4}}\right) \text {, } \\
& \hat{h}_{41}=\frac{1}{21} \sqrt{\frac{5}{2}}\left[\frac{\delta v^{3}}{20}(-1+2 \nu)+v^{2} \iota(1-3 \nu) e^{-i \Psi}\right]+\mathcal{O}\left(\frac{1}{c^{4}}\right), \\
& \hat{h}_{40}=\mathcal{O}\left(\frac{1}{c^{4}}\right) \text {, } \\
& \hat{h}_{55}=-\frac{625}{96 \sqrt{66}} \delta v^{3}(1-2 \nu)+\mathcal{O}\left(\frac{1}{c^{4}}\right) \text {, } \\
& \hat{h}_{54}=\mathcal{O}\left(\frac{1}{c^{4}}\right) \text {, } \\
& \hat{h}_{53}=-\frac{9}{32} \sqrt{\frac{3}{110}} v^{3} \delta(1-2 \nu)+\mathcal{O}\left(\frac{1}{c^{4}}\right), \\
& \hat{h}_{52}=\mathcal{O}\left(\frac{1}{c^{4}}\right) \text {, } \\
& \hat{h}_{51}=-\frac{1}{288 \sqrt{385}} \delta v^{3}(1-2 \nu)+\mathcal{O}\left(\frac{1}{c^{4}}\right) \text {, } \\
& \hat{h}_{50}=\mathcal{O}\left(\frac{1}{c^{4}}\right) \text {. }
\end{aligned}
$$

In Appendix B we display the modes $h_{22}, h_{33}$, and $h_{21}$ for generic inclination angle $\iota$. The modes $\hat{h}_{\ell m}$ for $m<0$ are derived from Eq. (4.17) by means of the relation $\hat{h}_{\ell-m}(\Phi)=(-1)^{\ell} \hat{h}_{\ell m}^{*}(\Phi+\pi)$. The non-precessing expressions are obtained by setting $\iota=0$ and $\alpha=\pi$. Notice that when $h_{\ell m}(\Phi)$ does not depend on $\Phi$, we have simply $\hat{h}_{\ell-m}=(-1)^{\ell} \hat{h}_{\ell m}^{*}$. For comparison with the modes of Refs. [27, 28] in the non-spinning case, it is important to be aware that the origin of the azimuthal angle there differs from ours by $-\pi / 2$, which produces an extra factor $(-i)^{m}$ (respectively $i^{m}$ ) with respect to us in the modes (respectively in the spin-weighted spherical harmonics).

Finally, let us emphasize that the $(\ell, m)$ modes defined by Eq. (4.5) depend on the particular choice of the source frame. In fact, they are functions of the spin and angular momentum components with respect to the $(\hat{\mathbf{x}}, \hat{\mathbf{y}}, \hat{\mathbf{z}})$ basis introduced in Sec. II] As there is no canonical way to fix the reference frame for precessing binaries because of the secular but perpetual variation of the direction $\mathbf{J} /|\mathbf{J}|$, it is important to be able to relate the $\hat{h}_{\ell m}$ 's given in Eq. (4.17) to the polarization modes $h_{\ell m}^{\prime}$ computed in another frame with different polarization vectors. Under a passive rotation

$$
R_{j}^{i}(A, B, \Gamma)=\left(\begin{array}{ccc}
\cos A & -\sin A & 0 \\
\sin A & \cos A & 0 \\
0 & 0 & 1
\end{array}\right)\left(\begin{array}{ccc}
\cos B & 0 & \sin B \\
0 & 1 & 0 \\
-\sin B & 0 & \cos B
\end{array}\right)\left(\begin{array}{ccc}
\cos \Gamma & -\sin \Gamma & 0 \\
\sin \Gamma & \cos \Gamma & 0 \\
0 & 0 & 1
\end{array}\right)
$$


the $(\ell, m)$ modes transform in the same way as they would in the case of a standard spherical harmonics decomposition [79, 80]. In fact, the spin-weighted -2 spherical harmonics are precisely devised to ensure this property for the modes of a spin-weighted -2 object [7]. The law of transformation for the $h_{\ell m}$ 's is given by

$$
h_{\ell m^{\prime}}^{\prime}\left(\Phi^{\prime}, \alpha^{\prime}, \iota^{\prime}, \chi_{n}^{\prime x^{\prime}}, \chi_{n}^{\prime y^{\prime}}, \chi_{n}^{\prime z^{\prime}}\right)=\sum_{m=-\ell}^{\ell} D_{m m^{\prime}}^{* \ell}(A, B, \Gamma) h_{\ell m}\left(\Phi, \alpha, \iota, \chi_{n}^{x}, \chi_{n}^{y}, \chi_{n}^{z}\right),
$$

where the primed quantities refer to the new frame and where $D_{m^{\prime} m}^{\ell}$ is the unitary Wigner matrix [7]

$$
D_{m^{\prime} m}^{\ell}(A, B, \Gamma)=(-1)^{m^{\prime}} \sqrt{\frac{4 \pi}{2 \ell+1}}-m^{\prime} Y^{\ell m}(B, A) e^{i m^{\prime} \Gamma}
$$

with the convention of Landau-Lifchitz [78]. The new angles read

$$
\begin{aligned}
& \iota^{\prime}=\arccos [\cos B \cos \iota-\cos (\Gamma+\alpha) \sin B \sin \iota] \\
& \alpha^{\prime}=\arccos \cos \alpha^{\prime}=\arccos \frac{\cos A[\cos \iota \sin B+\cos B \cos (\Gamma+\alpha) \sin \iota]-\sin A \sin \iota \sin (\Gamma+\alpha)}{\sqrt{1-(\cos B \cos \iota-\cos (\Gamma+\alpha) \sin B \sin \iota)^{2}}} \\
& \text { if } \cos \iota \sin A \sin B+\sin \iota[\cos B \cos (\Gamma+\alpha) \sin A+\cos A \sin (\Gamma+\alpha)] \geq 0, \\
& \alpha^{\prime}=2 \pi-\arccos \cos \alpha^{\prime} \quad \text { otherwise } \\
& \Phi^{\prime}=\arccos \cos \Phi^{\prime} \\
& =\arccos \frac{\cos B \cos \Phi \sin \iota-\sin B \sin \Gamma(\cos \iota \cos \Phi \sin \alpha+\cos \alpha \sin \Phi)+\cos \Gamma \sin B(\cos \iota \cos \alpha \cos \Phi-\sin \alpha \sin \Phi)}{\sqrt{1-(\cos B \cos \iota-\cos (\Gamma+\alpha) \sin B \sin \iota)^{2}}} \\
& \text { if } \cos \Phi \sin B \sin (\Gamma+\alpha)+(\cos \iota \cos (\Gamma+\alpha) \sin B+\cos B \sin \iota) \sin \Phi \geq 0, \\
& \Phi^{\prime}=2 \pi-\arccos \cos \Phi^{\prime} \quad \text { otherwise . }
\end{aligned}
$$

When the direction of the total angular momentum used to built the new frame coincides with that of $\mathbf{J}_{0}$, which results in the equality $\hat{\mathbf{z}}^{\prime}=\hat{\mathbf{z}}$, the Euler angle $B$ vanishes. Then, it can be checked from Eqs. (4.20d) and (4.20e) that $\Phi^{\prime}=\Phi$ as expected.

\section{FEATURES OF GRAVITATIONAL-WAVE MODES FOR PRECESSING BINARIES ON NEARLY CIRCULAR ORBITS}

We now study how spin effects change the waveform modes for generic precessing binaries. We consider two maximally spinning configurations with mass ratios 1:1 and 4:1. We label the spin configurations with the angles $\left\{\theta_{1}, \phi_{1}, \theta_{2}, \phi_{2}\right\}$, where $\left\{\theta_{i}, \phi_{i}\right\}$ describe the orientation of the spin vector of the $i^{\text {th }}$ body relative to the orbital angular momentum in the initial configuration, which we take to be a circular orbit with $M \omega_{\text {orb }}=0.001$. We use the full expressions for the $h_{\ell m}$ 's (i.e., the expressions that have not been expanded in $\iota$ ) as given in Appendix B and normalized following Eq. (4.16), but we replace $\Psi$ with $\Phi$.

After evolving through 1.5PN order all dynamical quantities they depend on [see Eqs. (3.11), (3.18), (3.20), and (3.21)], we compute the modulus - more often referred to as the absolute value — of a sample of modes. Considering the complicated structure of the $h_{\ell m}$ 's, their qualitative behavior in the presence of spins is discussed here in terms of the $\iota$-expanded formulae (4.17). Let us focus on two spin configurations. The configuration $\operatorname{Spin}_{\mathrm{A}}=\{\pi / 2,0, \pi / 2, \pi / 2\}$ has both spin vectors in the orbital plane, meaning a relatively large inclination angle. The configuration Spin $\mathrm{B}=$ $\{\pi / 6, \pi / 4, \pi / 6, \pi\}$ has a smaller component of total spin transverse to the orbital angular momentum, hence a smaller inclination angle. In Figs. 3 and 4 we plot the amplitude of the $\hat{h}_{\ell m}$ over the frequency range $2 M \dot{\Phi}=0.02-0.15$, the upper frequency being reached roughly 2 cycles before merger, for an equal-mass, non-spinning binary [70]. For a binary of total mass $16 M_{\odot}$, the dominant second harmonic varies over the frequency range 40-300 Hz. For a $6.5 \times 10^{6} M_{\odot}$ binary, this range is shifted to $10^{-4}-7.5 \times 10^{-4} \mathrm{~Hz}$.

The left panel of Fig. 2 shows the inclination angle $\iota$ as a function of the dimensionless frequency $2 M \dot{\Phi}$. We see that the inclinations are much larger in the case of 4:1 mass-ratio than for equal masses. Moreover, the inclination increases monotonically in the equal-mass case, whereas the 4:1 mass ratio exhibits nutation, since the inclination oscillates, but grows on average. These observations can be explained as follows. At early times, when the binary has a large orbital separation, we have $|\mathbf{L}| \gg|\mathbf{S}|$, where $\mathbf{S}=\mathbf{S}_{1}+\mathbf{S}_{2}$ is the total spin, so that $\mathbf{J}=\mathbf{L}+\mathbf{S}$ and $\mathbf{L}$ are 

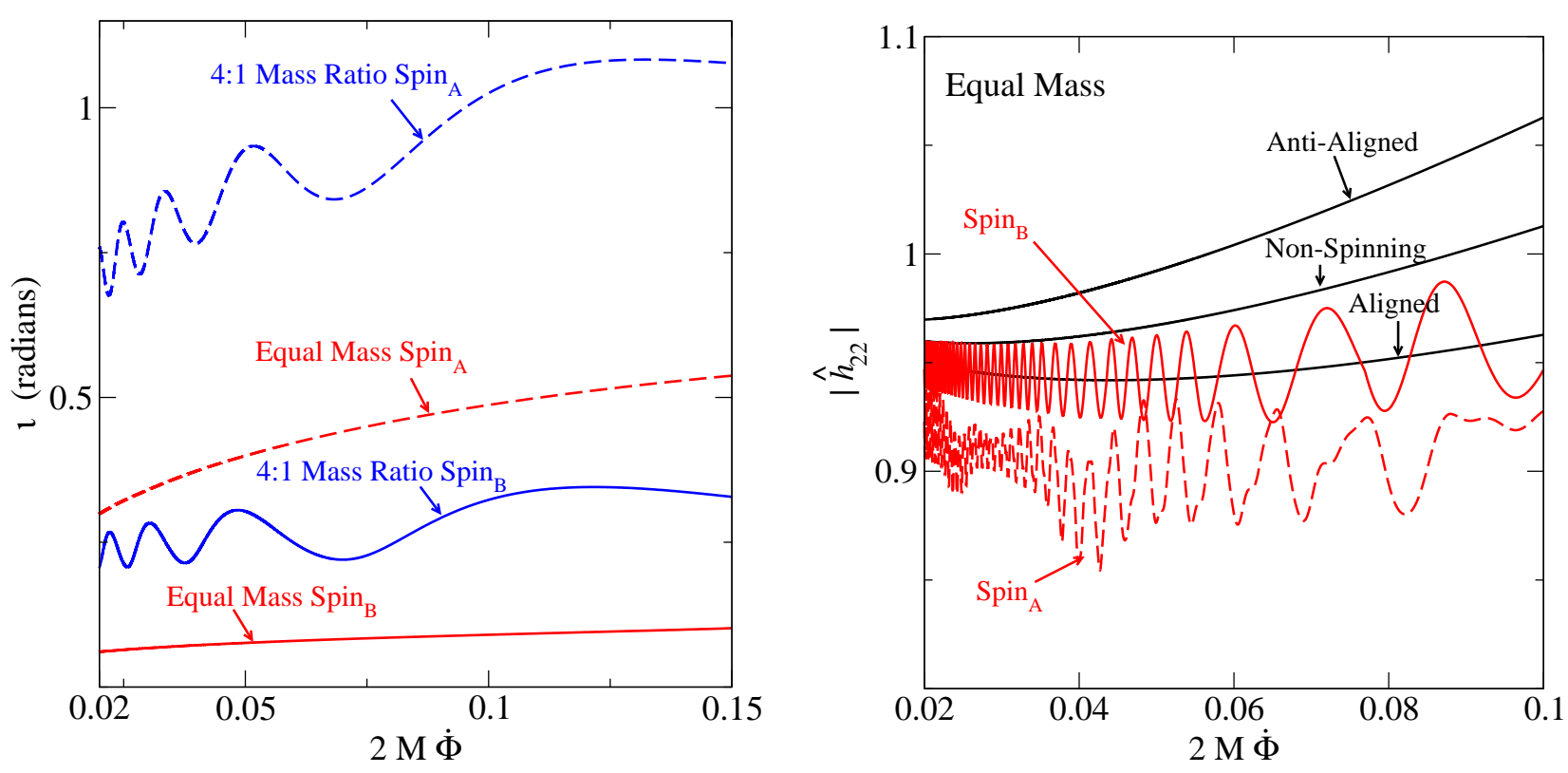

FIG. 2: The left panel shows the inclination angle of the orbital angular momentum relative to the total angular momentum, $\iota$, as a function of $2 M \dot{\Phi}$ for binaries with mass ratios $1: 1$ and $4: 1$, having initial spin orientations relative to the orbital angular momentum: $\operatorname{Spin}_{\mathrm{A}}=\left\{\theta_{1}=\pi / 2, \phi_{1}=0, \theta_{2}=\pi / 2, \phi_{2}=\pi / 2\right\}$ and $\operatorname{Spin}_{\mathrm{B}}=\left\{\theta_{1}=\pi / 6, \phi_{1}=\pi / 4, \theta_{2}=\pi / 6, \phi_{2}=\pi\right\}$. The right panel compares the modulus of $h_{22}$ for the two precessing spin configurations with the non-spinning, aligned and anti-aligned cases for equal masses. The computations use waveforms accurate to $1.5 \mathrm{PN}$ in amplitude and phase evolved with the precession equations at 1.5PN order [see Eqs. (3.11), (3.18), (3.20), and (3.21)]. Note that these plots (and those of Figs. 3 and 4) begin at $2 M \dot{\Phi}=0.02$ which is approximately where the dominant second harmonic from a binary of total mass $16 M_{\odot}$ enters the LIGO band at $40 \mathrm{~Hz}$ and where the second harmonic from a binary of total mass $6.5 \times 10^{6} M_{\odot}$ enters the LISA band at $10^{-4}$ $\mathrm{Hz}$.

nearly aligned. Radiation reaction causes $|\mathbf{L}|$ to decrease, making $\mathbf{J}$ move away from $\mathbf{L}$ and toward $\mathbf{S}$. This is why the inclination angle, $\iota$, grows on average as the frequency increases. The absence of oscillations for the inclination angle $\iota$ in the equal-mass case can be explained by the fact that we are evolving the dynamics, in particular the precession equations (3.18), through 1.5PN order, i.e., we are neglecting spin-spin effects. Due to the equality $\boldsymbol{\Omega}_{1}=\boldsymbol{\Omega}_{2}$ at this accuracy level, the precession equations simplify then to a single equation of the form $d \mathbf{S} / d t=\boldsymbol{\Omega} \times \mathbf{S}$. In the absence of radiation reaction, $\mathbf{S}$ precesses around a fixed direction with a constant frequency, and the inclination is constant (apart from the increase produced by radiation reaction). For unequal masses this symmetry does not exist, with the consequence that one must solve two coupled equations for $\mathbf{S}_{1}, \mathbf{S}_{2}$ instead of a single equation for $\mathbf{S}$. The motion of the spin vectors is thus more complicated. Schematically, they rotate about a fixed direction while also bobbing up and down [81.

The right panel of Fig. 2 plots, for the case of equal masses, the absolute value of the $h_{22}$ mode normalized to its Newtonian order expression, $\hat{h}_{22}$, for both precessing spin configurations as well as the non-spinning, aligned and anti-aligned cases. One interesting feature is that the aligned and anti-aligned cases do not bound the absolute value of the modes for generic spin configurations. This and other features of the plot can be understood from the $\iota$ expansion (4.17a) of $\hat{h}_{22}$, which contains four spin corrections. The first correction, $-\iota^{2} / 2$, is zero for the aligned and anti-aligned cases, while it decreases the absolute value of the $\hat{h}_{22}$ mode for all other spin configurations. If $\iota$ is comparable to 1 radian, it can be a significant correction. The second correction, $(1 / 3) e^{i \Psi} \delta v \iota$, vanishes for equal masses. For unequal masses, it interferes with the non-spinning terms and, because it has a different dependence on the orbital phase, produces oscillations in the absolute value of $\hat{h}_{22}$. Next, the $1 \mathrm{PN}$ order spin correction, $-\left(v^{2} / 2\right) e^{i(\alpha+\Psi)}\left[\chi_{a}^{x}-i \chi_{a}^{y}+\delta\left(\chi_{s}^{x}-i \chi_{s}^{y}\right)\right]$, generates oscillations that depend on the spin vector components transverse to the total angular momentum. Finally, the $1.5 \mathrm{PN}$ order spin correction, $v^{3}\left[-4 \delta \chi_{a}^{z} / 3+(4 / 3)(-1+\nu) \chi_{s}^{z}\right]$, lowers (raises) the absolute value of $\hat{h}_{22}$ for spins aligned (anti-aligned) with the total angular momentum. It is solely responsible for the spread between the aligned and anti-aligned cases, as the other corrections all vanish then.

A similar analysis can be applied to understand the behavior of the other modes. As an illustration, we plot in Fig. 3 all of the $\ell=2$ modes for mass ratios 1:1 and 4:1. The $\hat{h}_{21}$ mode 4.17b is zero for non-spinning equal mass binaries. However, it contains several spin corrections and can have significant amplitude for precessing binaries, particularly for large $\iota$. It can exhibit complicated modulation, as its different spin corrections interfere with one another. The 

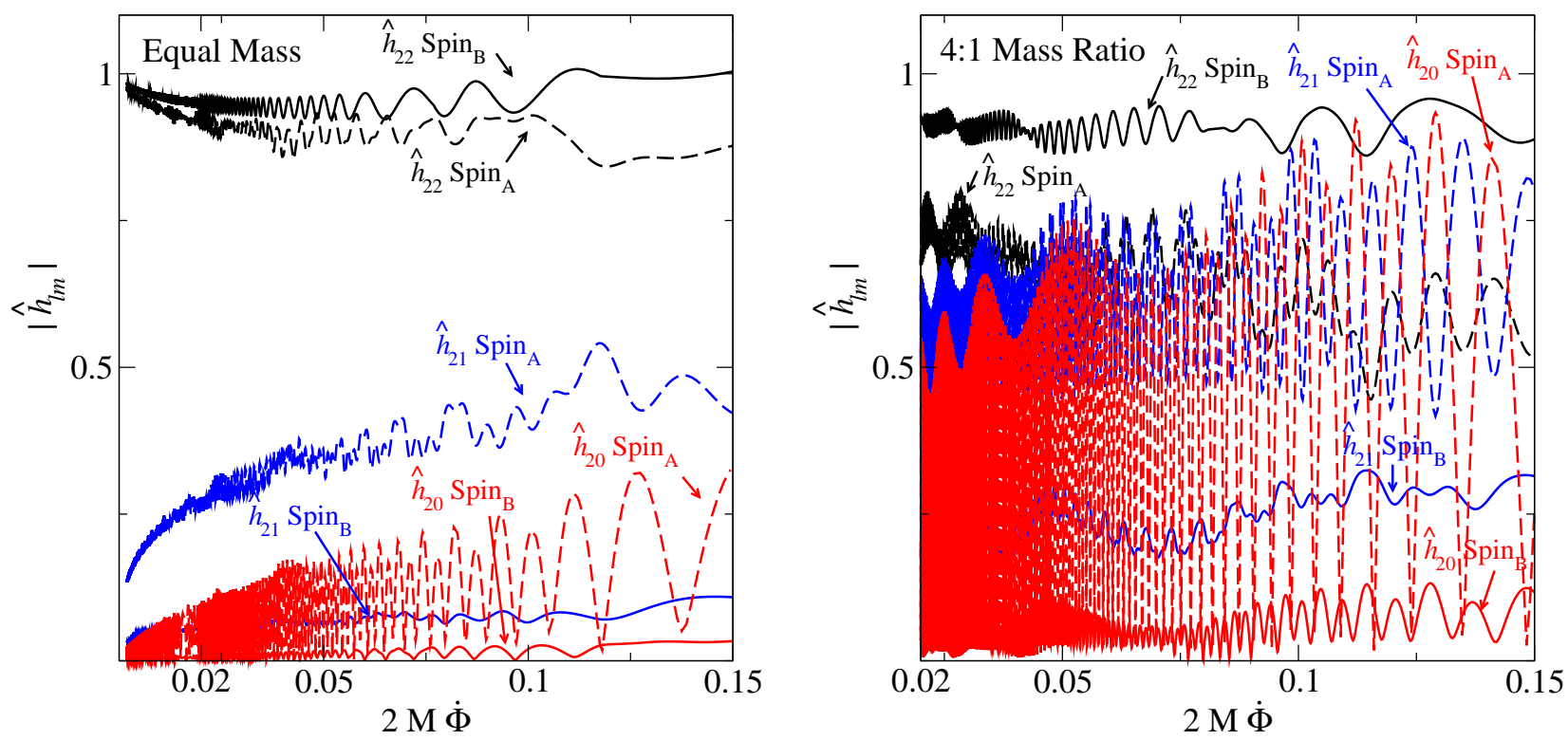

FIG. 3: We plot the modulus of the $\ell=2$ modes for mass ratios 1:1 (left panel) and 4:1 (right panel) with the spin configurations described in Fig. 2. The computations use waveforms accurate to 1.5PN order in amplitude and phase evolved with precession equations at $1.5 \mathrm{PN}$ order. The dashed lines are the larger $\iota$ configuration $\left(\mathrm{Spin}_{\mathrm{A}}\right)$ and the solid lines are the smaller $\iota$ configuration $\left(\operatorname{Spin}_{\mathrm{B}}\right)$. We see that as $\iota$ increases, the modulus of $\hat{h}_{22}$ decreases while the modulus of the other $\ell=2$ modes increases. This effect becomes more pronounced when the mass ratio is more extreme.

$\hat{h}_{20}$ mode (4.17c) also has several spin corrections, most notably $2 \iota^{2} \cos \Psi$. This correction is responsible for the large oscillations in the absolute value of $\hat{h}_{20}$. Note that in the late stages of the inspiral evolution for the 4:1 mass-ratio case, where $\iota \sim 1$ radian, these oscillations in the absolute value of $\hat{h}_{20}$ peak near the absolute value of $\hat{h}_{22}$. The other spin corrections in $\hat{h}_{20}$ are responsible for the further modulations of the absolute value.

Figure 4 plots the absolute value of the $\ell=3$ and $\ell=4$ modes for equal masses. Note that these modes are about two orders of magnitude smaller than the $\ell=2$ modes. This remains true in the non-spinning case for the mass ratios we consider. In the case of non-spinning, equal mass binaries, for $\ell=3$ only the $\hat{h}_{32}$ mode 4.17e is non-zero. The $1.5 \mathrm{PN}$ order spin correction decreases (increases) this mode's absolute value if the spins are aligned (anti-aligned) with the total angular momentum, in a similar way as in the $\hat{h}_{22}$ mode. For unequal masses, the $\hat{h}_{32}$ mode (4.17e) also has an interference term proportional to $\iota$. The other $\ell=3$ modes are non-zero for generic precessing binaries, and generally have larger absolute values for larger inclinations. In the left panel of Fig. 固 we indeed observe that $\left|\hat{h}_{33}\right|$, $\left|\hat{h}_{31}\right|$, and $\left|\hat{h}_{30}\right|$ are greater for the configuration $\operatorname{Spin}_{\mathrm{A}}$ than for $\operatorname{Spin}_{\mathrm{B}}$. For $\ell=4$, only the $\hat{h}_{44}$ and $\hat{h}_{42}$ modes (4.17h), 4.17j) are non-zero for non-spinning, equal-mass binaries, with $\hat{h}_{44}$ being the largest. Though the $\iota$-expanded form of the $\hat{h}_{44}, \hat{h}_{42}$ and $\hat{h}_{40}$ modes in Eq. (4.17) do not have any spin corrections through $\mathcal{O}\left(1 / c^{3}\right)$, we do see spin effects in all of the $\ell=4$ modes when we plot the full expressions accurate through $v^{3}$. The $v^{2}$ and $v^{3}$ coefficients in the full expressions for the $\hat{h}_{44}, \hat{h}_{42}$ and $\hat{h}_{40}$ modes depend on the inclination $\iota$ but not on the spin vector components and this dependence is such that if we treat $\iota$ as a $1 / c$ correction by performing a Taylor expansion in powers of $\iota$, then the spin terms are proportional to $v^{2} \iota^{2}, v^{3} \iota$ and higher order in $\iota$. They are thus considered as higher order corrections in the $\iota$ expansion, though they are present when we expand only in powers of $v$. None of the $\ell=4$ modes contain any spin corrections proportional to the spin vector components through order $v^{3}$. Those corrections would appear only at higher order in $v$.

While we have plotted the $\ell=3$ and $\ell=4$ modes solely for equal masses, we have also studied these modes for 4:1 mass-ratio binaries. We find that they are affected by the change of mass ratio in much the same way as the $\ell=2$ modes: the redistribution of signal among the $\ell=3$ and $\ell=4$ modes is more pronounced for asymmetric binaries than for equal mass binaries. However, even for 4:1 binaries with large $\iota\left(\operatorname{Spin}_{\mathrm{A}}\right)$ spin configuration, all of the $\ell=3$ and $\ell=4$ modes are still one or two orders of magnitude smaller than $\hat{h}_{22}$, whereas the $\hat{h}_{21}$ and $\hat{h}_{20}$ modes can be comparable to $\hat{h}_{22}$. The reason is essentially that $\hat{h}_{21}$ and $\hat{h}_{20}$ have $\iota$ corrections at leading order in $v$, while $\iota$ corrections to the $\ell=3$ and $\ell=4$ modes all appear at higher order, and so they do not have as strong an effect as for $\hat{h}_{21}$ and $\hat{h}_{20}$.

By examining the absolute values of the $\hat{h}_{\ell m}$ modes for precessing binaries, we see that they can be significantly 

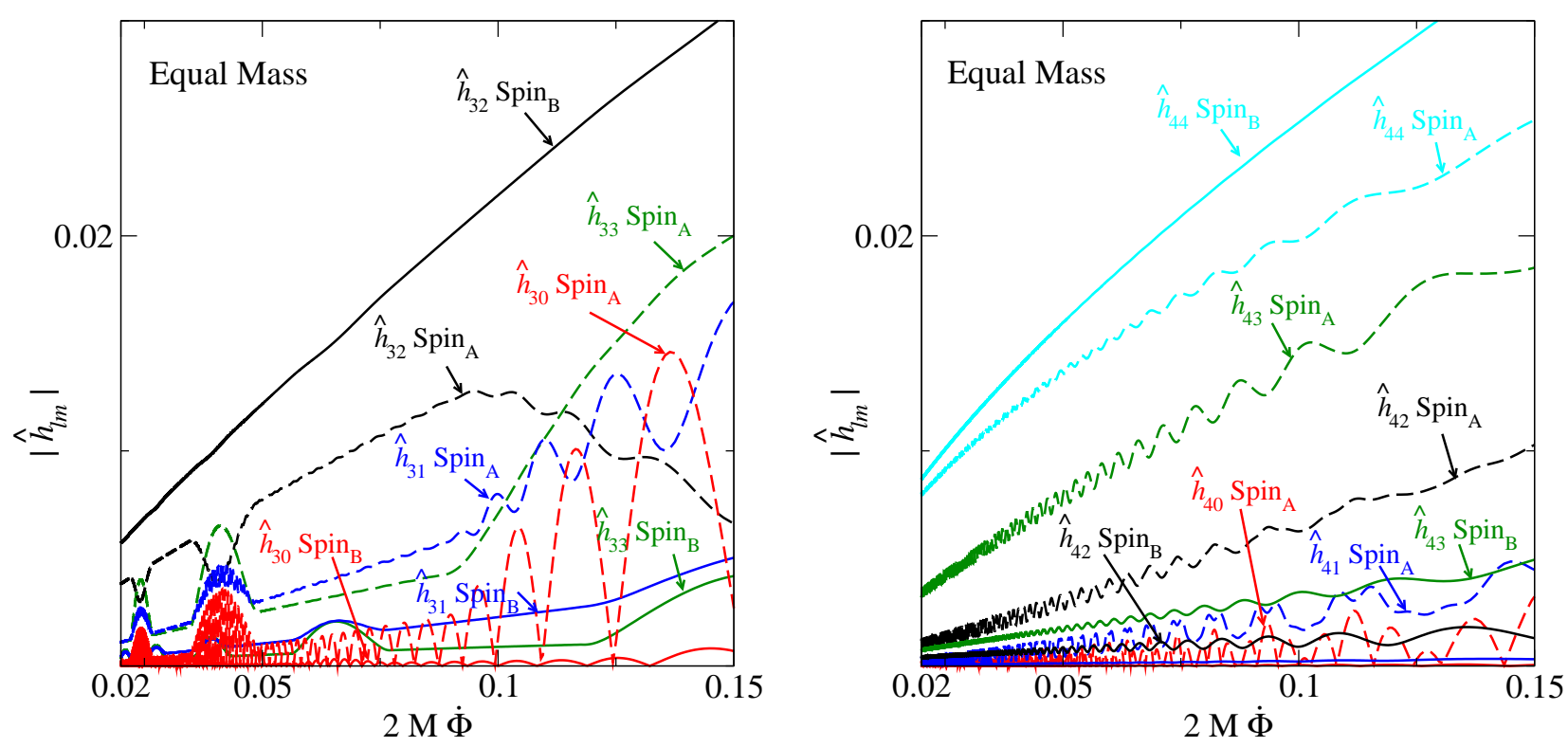

FIG. 4: We plot the modulus of the $\ell=3$ modes (left panel) and $\ell=4$ modes (right panel) for equal masses with the spin configurations described in Fig. 2 The computations use waveforms accurate to $1.5 \mathrm{PN}$ order in both amplitude and phase evolved by means of the $1.5 \mathrm{PN}$ precession equations. The dashed lines refer to the larger $\iota$ configuration $\left(\mathrm{Spin}_{\mathrm{A}}\right)$, the solid lines to the smaller iota configuration $\left(\operatorname{Spin}_{\mathrm{B}}\right)$. We see a redistribution of power among the modes similar to Fig. 3 . As $\iota$ increases, the largest modes for the non-precessing cases $\left(\left|\hat{h}_{32}\right|\right.$ and $\left.\left|\hat{h}_{44}\right|\right)$ become smaller, while the other modes become larger.

altered by the motion of the orbital plane relative to the frame used to perform the mode decomposition and the signal may be redistributed among the modes as observed in Ref. [80]. This suggests that all modes, not just the dominant ones for non-spinning binaries, are needed to accurately describe the waveforms emitted from precessing binary systems, especially for asymmetric binaries and binaries with large inclinations where this redistribution of signal among the modes is most dramatic.

Let us close this section with a few comments about the applicability of the full and $\iota$-expanded expressions for the modes and the possibility of combining them with higher order non-spinning corrections. We find that the absolute values of the full and $\iota$-expanded $\hat{h}_{\ell m}$ modes are often quite close to each other for relatively small $\iota$. For inclinations less than half a radian $\left(30^{\circ}\right)$, the difference in $\left|\hat{h}_{22}\right|$ is typically of a few percent. For $\iota$ comparable to or larger than a radian $\left(60^{\circ}\right)$, significant differences between the full and $\iota$-expanded modes develop and the absolute values may differ by $\sim 10-100 \%$ when $\iota \geq 1$ radian. Nonetheless, the $\iota$-expanded modes are very useful in understanding the qualitative behavior of precessing binaries, even for inclinations $\sim 1$ radian, albeit they should not be used for precise quantitative studies of binaries with large inclination angles.

In Refs. [27, 28], expressions of the modes are given to 3PN order for non-spinning binaries. We have compared their absolute values to that of the corresponding quantities truncated at $1.5 \mathrm{PN}$ order, which has shown us that they typically differ by $\sim 1-10 \%$. For example, the absolute value of the $1.5 \mathrm{PN}$ and $3 \mathrm{PN}$ order $h_{22}$ modes for equal-mass binaries differ by less than $1 \%$ at $2 M \dot{\Phi}=0.01$ and by about $3 \%$ at $2 M \dot{\Phi}=0.05$ or $2 M \dot{\Phi}=0.12$. The other modes typically have a larger difference. The $1.5 \mathrm{PN}$ order and highest known order absolute values for $h_{32}, h_{44}$ and $h_{42}$ differ by about $5-15 \%$ over this same frequency range for equal mass binaries. For 4:1 mass ratio binaries, the differences in absolute value are similar.

Known higher-order non-spinning terms can actually be included in the amplitude if enough care is taken. In the non-precessing case, the modes $h_{\ell m}$ are proportional to $e^{-i m \Psi}$, as in the non-spinning case, because $\hat{\mathbf{n}}$ and $\hat{\boldsymbol{\lambda}}$ appearing in the strain tensor $h_{i j}$ are trigonometric functions of the orbital phase $\Phi$. In constructing the $h_{\ell m}$ 's from $h_{i j}$, they generate an exponential dependence on multiples of $\Phi$. However, for the case of a precessing binary with small $\iota, \hat{\mathbf{n}}$ and $\hat{\boldsymbol{\lambda}}$ are trigonometric functions of $\Phi+\alpha$. Thus, the $h_{\ell m}$ 's contain then all of the non-spinning terms, but with the substitution $\Psi \rightarrow \Psi+\alpha$. The situation is different for the general precessing case. The vectors $\hat{\mathbf{n}}$ and $\hat{\boldsymbol{\lambda}}$ depend on $\Phi, \alpha$, and $\iota$, and the resulting $h_{\ell m}$ have a complicated dependence on all three of these quantities that cannot be simply related to the non-spinning case. These considerations show that it is only for binaries with a small inclination (or no inclination) that we can readily construct the $h_{\ell m}$ 's with spin effects up to $1.5 \mathrm{PN}$ order and non-spinning corrections up to 3PN order. For spins (anti-)aligned it is trivial to add the higher-order non-spinning corrections of Refs. 27, 28] to the $h_{\ell m}$ given in Eq. [4.17). For precessing binaries with small inclinations, they can 
be added to our expressions in Eq. (4.17) with the substitution $\Psi \rightarrow \Psi+\alpha$. For general precessing binaries, it is not so simple to include higher-order non-spinning corrections to the full expressions for the $h_{\ell m}$ given in Appendix B. To do this properly, we would need the spin terms at the same order as the non-spinning terms and repeat the derivation of the $h_{\ell m}$ to a higher order.

\section{READY-TO-USE FREQUENCY-DOMAIN TEMPLATES FOR SPINNING, NON-PRECESSING BINARIES}

\section{A. Gravitational-wave polarizations in time domain}

In the non-precessing case, the orbital angular momentum points in a fixed direction which we take to be the $z$-axis (see Fig. 1) and the spins are either aligned or anti-aligned with it. The basis vectors of the orbital plane, $\hat{\mathbf{x}}_{L}(t)$ and $\hat{\mathbf{y}}_{L}(t)$, are constant in time. They can be freely chosen to be any pair of orthogonal unit vectors in the $x-y$ plane. Here, following the convention of Ref. [25], we choose $\hat{\mathbf{x}}_{L}=\hat{\mathbf{P}}=\hat{\mathbf{N}} \times \hat{\mathbf{L}}_{\mathrm{N}} /\left|\hat{\mathbf{N}} \times \hat{\mathbf{L}}_{\mathrm{N}}\right|$, so that the phase is zero at the ascending node (where the orbital separation vector crosses the plane of the sky from below). This is equivalent to setting $\iota=0$ and $\alpha=\pi$ in Eqs. (3.12), (A2), and (A3). Note also that since the orbital plane remains fixed, the phase $\Phi$ defined through Eq. (3.10) coincides with the standard definition of the orbital phase, that is

$$
\omega_{\text {orb }}=\dot{\Phi} \text {. }
$$

In the non-precessing case, the vectors in terms of which the GW polarizations are expressed originally take the simpler form

$$
\begin{aligned}
\hat{\mathbf{n}} & =(\sin \Phi,-\cos \Phi, 0), \\
\hat{\boldsymbol{\lambda}} & =(\cos \Phi, \sin \Phi, 0), \\
\hat{\mathbf{N}} & =(\sin \theta, 0, \cos \theta) \\
\hat{\mathbf{L}}_{\mathrm{N}} & =(0,0,1)
\end{aligned}
$$

By plugging the expressions (6.2) -6.5) into Eq. (3.3) and taking the combinations given in Eq. (2.1), we obtain an equation similar to Eq. (3.12). The spin-dependent $1 \mathrm{PN}, 1.5 \mathrm{PN}$ and $2 \mathrm{PN}$ order polarization coefficients read

$$
\begin{aligned}
H_{+}^{(1, \mathrm{SO})} & =v^{2} s_{\theta} \cos \Psi\left[\chi_{a} \cdot \hat{\mathbf{L}}_{\mathrm{N}}+\delta \boldsymbol{\chi}_{s} \cdot \hat{\mathbf{L}}_{\mathrm{N}}\right] \\
H_{+}^{(3 / 2, \mathrm{SO})} & =v^{3} \cos 2 \Psi \frac{4}{3}\left[\left(1+c_{\theta}^{2}\right)\left(\chi_{s} \cdot \hat{\mathbf{L}}_{\mathrm{N}}+\delta \boldsymbol{\chi}_{a} \cdot \hat{\mathbf{L}}_{\mathrm{N}}\right)+\nu\left(1-5 c_{\theta}^{2}\right) \boldsymbol{\chi}_{s} \cdot \hat{\mathbf{L}}_{\mathrm{N}}\right], \\
H_{+}^{(2, \mathrm{SS})} & =-v^{4} 2 \nu\left(1+c_{\theta}^{2}\right)\left(\boldsymbol{\chi}_{s}^{2}-\boldsymbol{\chi}_{a}^{2}\right) \cos 2 \Psi \\
H_{\times}^{(1, \mathrm{SO})} & =v^{2} s_{\theta} c_{\theta} \sin \Psi\left[\chi_{a} \cdot \hat{\mathbf{L}}_{\mathrm{N}}+\delta \chi_{s} \cdot \hat{\mathbf{L}}_{\mathrm{N}}\right] \\
H_{\times}^{(3 / 2, \mathrm{SO})} & =v^{3} \sin 2 \Psi \frac{4}{3} c_{\theta}\left[2\left(\chi_{s} \cdot \hat{\mathbf{L}}_{\mathrm{N}}+\delta \boldsymbol{\chi}_{a} \cdot \hat{\mathbf{L}}_{\mathrm{N}}\right)-\nu\left(1+3 c_{\theta}^{2}\right) \boldsymbol{\chi}_{s} \cdot \hat{\mathbf{L}}_{\mathrm{N}}\right], \\
H_{\times}^{(2, \mathrm{SS})} & =-v^{4} 4 \nu c_{\theta}\left(\chi_{s}^{2}-\chi_{a}^{2}\right) \sin 2 \Psi
\end{aligned}
$$

where SS labels the $\operatorname{spin}(1)-\operatorname{spin}(2)$ contributions. ${ }^{9}$ In the equations above, we use the shorthand $c_{\theta}=\cos \theta$ and $s_{\theta}=\sin \theta$. Note also that the phase $\Psi$ is the shifted orbital phase that relates to $\Phi$ at our accuracy level as

$$
\Psi=\Phi-2 v^{3}\left(1-\frac{\nu}{2} v^{2}\right) \ln \left(\frac{\omega_{\text {orb }}}{\omega_{0}}\right),
$$

where $\omega_{0}$ can be chosen arbitrarily. Expressed in terms of the orbital phase $\Phi$, the GW polarizations would contain terms logarithmic in $\omega_{\text {orb }}$, arising from the propagation of the tails. However, introducing the phase (6.6g), they are all absorbed (up to the 2.5PN order we are considering) into the phase variable [25].

The spin-dependent polarizations (6.6a) (6.6f) were derived in Ref. 35] [see Eqs. (F24a)-(F25c) in that paper], although the $1.5 \mathrm{PN}$ and $2 \mathrm{PN}$ order cross polarizations had an erroneous sign, which is corrected here.

\footnotetext{
${ }^{9}$ We remind that $\operatorname{spin}(1)$-spin(1) and $\operatorname{spin}(2)$-spin(2) effects in the waveform polarizations are currently unknown.
} 


\section{B. Spin-orbit effects at $1.5 \mathrm{PN}$ order and spin-spin effects at $2 \mathrm{PN}$ order in the frequency-domain gravitational-wave amplitude}

Writing $h(t)=h_{+} F_{+}+h_{\times} F_{\times}$and collecting terms by PN order and by sines or cosines of harmonics of the orbital frequency, we can write the time-domain strain in the compact form:

$$
\begin{aligned}
h(t) & =\frac{2 M \nu}{D_{L}} \sum_{n=0}^{5} \sum_{k=1}^{7} V_{k}^{2+n}\left[\alpha_{k}^{(n)} \cos (k \Psi(t))+\beta_{k}^{(n)} \sin (k \Psi(t))\right]+\mathcal{O}\left(v^{8}\right), \\
& =\frac{2 M \nu}{D_{L}} \sum_{n=0}^{5} \sum_{k=1}^{7} V_{k}^{2+n}\left[\alpha_{k}^{(n)} \cos (k \Psi(t))+\beta_{k}^{(n)} \cos \left(k \Psi(t)-\frac{\pi}{2}\right)\right]+\mathcal{O}\left(v^{8}\right),
\end{aligned}
$$

where $n / 2$ is the PN order and $k$ labels the harmonics of the orbital phase. The PN expansion parameter is defined as $V=(2 \pi M F)^{1 / 3}$, with $F=\omega_{\text {orb }} /(2 \pi)$. We shall denote the GW frequency by $f$. For the $k^{\text {th }}$ harmonic, we have then the relation $f=f_{k} \equiv k F$, so that

$$
V_{k}=\left(2 \pi M \frac{f_{k}}{k}\right)^{1 / 3}
$$

Given a function of the form $h(t)=A(t) \cos \phi(t)$, where $\phi(t)$ is a monotonically increasing function satisfying $d \ln \mathrm{A}(\mathrm{t}) / d t \ll d \phi(t) / d t$, we can compute its Fourier transform by applying the stationary-phase approximation (SPA):

$$
\tilde{h}(f) \simeq \frac{1}{2} A(t(f)) \sqrt{\frac{2 \pi}{\ddot{\phi}(t(f))}} e^{i(2 \pi f t(f)-\phi(t(f))-\pi / 4)},
$$

$t(f)$ being defined here for each frequency $f$ as the value of $t$ for which $(d \phi / d t)(t)=2 \pi f$. In a similar manner, we apply the SPA to each term in the sum of Eq. 6.7). Moreover, for each harmonic of the orbital phase, we expand the factor inversely proportional to the second time derivative of the orbital phase entering Eq. (6.9) in a PN series of the form

$$
\sqrt{\frac{2 \pi}{k \ddot{\phi}}}=\left(k \frac{d F}{d t}\right)^{-1 / 2}=\sqrt{\frac{5 \pi}{k 48 \nu}} M V_{k}^{-11 / 2}\left[1+\mathcal{S}_{2} V_{k}^{2}+\mathcal{S}_{3} V_{k}^{3}+\mathcal{S}_{4} V_{k}^{4}+\mathcal{S}_{5} V_{k}^{5}+\mathcal{O}\left(V_{k}^{6}\right)\right],
$$

with

$$
\begin{aligned}
& \mathcal{S}_{2}=\frac{743}{672}+\frac{11}{8} \nu \\
& \mathcal{S}_{3}=-2 \pi+\left(\frac{113}{24}-\frac{19}{6} \nu\right) \chi_{s} \cdot \hat{\mathbf{L}}_{\mathrm{N}}+\frac{113}{24} \delta \boldsymbol{\chi}_{a} \cdot \hat{\mathbf{L}}_{\mathrm{N}} \\
& \mathcal{S}_{4}=\frac{7266251}{8128512}+\frac{18913}{16128} \nu+\frac{1379}{1152} \nu^{2}-\nu\left(\frac{721}{96}\left(\left(\chi_{s} \cdot \hat{\mathbf{L}}_{\mathrm{N}}\right)^{2}-\left(\chi_{a} \cdot \hat{\mathbf{L}}_{\mathrm{N}}\right)^{2}\right)-\frac{247}{96}\left(\chi_{s}^{2}-\chi_{a}^{2}\right)\right), \\
& \mathcal{S}_{5}=\pi\left(-\frac{4757}{1344}+\frac{57}{16} \nu\right) .
\end{aligned}
$$

The expansion (6.10) without spin corrections in the amplitude was first given in Ref. [48]. We have added to it the leading order $\mathrm{SO}$ corrections through 1.5PN order and the $\operatorname{spin}(1)-\operatorname{spin}(2) \mathrm{SS}$ corrections appearing at $2 \mathrm{PN}$ order. In principle, SO corrections at 2.5PN order and $\operatorname{spin}(1)-\operatorname{spin}(1), \operatorname{spin}(2)-\operatorname{spin}(2) \mathrm{SS}$ corrections at $2 \mathrm{PN}$ arising from the spin contribution to the orbital frequency are also present. However, when calculating spin terms in the frequencydomain amplitude, we neglect them because they have not been calculated yet beyond the 1.5PN order in the time domain amplitude. The spin contribution at $2 \mathrm{PN}$ and $2.5 \mathrm{PN}$ order to the Fourier domain amplitude is not complete unless we take both into account.

Defining the frequency-dependent SPA phase as

$$
\Psi_{\mathrm{SPA}}(f)=2 \pi f t(f)-\Psi(f)
$$

the frequency domain waveform with amplitude corrections containing SO effects through 1.5PN order and spin(1)$\operatorname{spin}(2)$ effects through $2 \mathrm{PN}$ order is

$$
\tilde{h}(f)=\frac{M \nu}{D_{L}} \sum_{n=0}^{5} \sum_{k=1}^{7} V_{k}^{2+n}\left(k \frac{d F}{d t}\right)^{-1 / 2}\left(\alpha_{k}^{(n)} e^{i(2 \pi f t(F)-k \Psi(F)-\pi / 4)}+\beta_{k}^{(n)} e^{i(2 \pi f t(F)-(k \Psi(F)-\pi / 2)-\pi / 4)}\right),
$$




$$
\begin{aligned}
& =\frac{M \nu}{D_{L}} \sum_{n=0}^{5} \sum_{k=1}^{7} V_{k}^{n-\frac{7}{2}} \sqrt{\frac{5 \pi}{k 48 \nu}} M\left(1+\mathcal{S}_{2} V_{k}^{2}+\mathcal{S}_{3} V_{k}^{3}+\mathcal{S}_{4} V_{k}^{4}+\mathcal{S}_{5} V_{k}^{5}\right)\left(\alpha_{k}^{(n)}+e^{i \pi / 2} \beta_{k}^{(n)}\right) e^{i\left(k \Psi_{\mathrm{SPA}}(f / k)-\pi / 4\right)} \\
& =\frac{M^{2}}{D_{L}} \sqrt{\frac{5 \pi \nu}{48}} \sum_{n=0}^{5} \sum_{k=1}^{7} V_{k}^{n-\frac{7}{2}} \mathcal{C}_{k}^{(n)} e^{i\left(k \Psi_{\mathrm{SPA}}(f / k)-\pi / 4\right)}
\end{aligned}
$$

with

$$
\mathcal{C}_{k}^{(n)}=\frac{1}{\sqrt{k}}\left(\alpha_{k}^{(n)}+i \beta_{k}^{(n)}\right)+\sum_{m=2}^{n} \frac{\mathcal{S}_{m}}{\sqrt{k}}\left(\alpha_{k}^{(n-m)}+i \beta_{k}^{(n-m)}\right)
$$

where the index $n$ denotes the PN order and the index $k$ the harmonics. Explicit expressions for the $\mathcal{C}_{k}^{(n)}$ can be found in Appendix D. The non-spinning terms in the amplitude agree with Ref. [47], although we have written them in a different, more explicit manner. Notice that recently the non-spinning amplitude corrections were calculated through $3 \mathrm{PN}$ order [28], but in this paper we restricted the computation to $2.5 \mathrm{PN}$ order.

\section{Spin-orbit effects at $2.5 \mathrm{PN}$ order in the frequency-domain gravitational-wave phase}

For matched filtering, it is best to know the GW phasing at the highest PN order. We now derive the SO contributions to the SPA phase through 2.5PN and the SS contributions (including spin(1)-spin(1) and $\operatorname{spin}(2)-\operatorname{spin}(2)$ contributions) to the SPA phase through $2 \mathrm{PN}$ order.

The PN expansion of the SPA phase $\Psi_{\mathrm{SPA}}(F)$ can be obtained from the PN expansions of the binary center-of-mass energy, $E$, and GW flux, $F$, via the energy balance equation

$$
-\frac{d E}{d t}=\mathcal{F}
$$

Using $d \Psi / d t=2 \pi F=v^{3} / M$, we can re-write the energy balance equation as the differential equations

$$
d t=-\frac{d E}{d v} \frac{1}{\mathcal{F}} d v
$$

and

$$
d \Psi=-\frac{d E}{d v} \frac{1}{\mathcal{F}} \frac{v^{3}}{M} d v
$$

The quantities $E$ and $\mathcal{F}$ are known as power series in $v=(2 \pi M F)^{1 / 3}$. The non-spinning terms in the expansions of $E$ and $\mathcal{F}$ have been calculated by Refs. 29, 82 [86], while the spin contributions to these quantities through 2.5PN order were derived by Refs. [22, 23, 30, 34, 35, 75]. The center-of-mass energy and the flux read

$$
\begin{aligned}
& E(v)=E_{\text {Newt }} v^{2}\left(1+\sum_{i=2}^{6} E_{i} v^{i}\right), \\
& \mathcal{F}(v)=F_{\text {Newt }} v^{10}\left(1+\sum_{i=2}^{7} F_{i} v^{i}\right),
\end{aligned}
$$

where the coefficients $E_{i}$ and $F_{i}$ are explicitly given in Appendix C By inserting Eqs. (6.18), (6.19) into Eqs. (6.16), (6.17), we obtain rational function approximations to the integrands. We then find the Taylor series of the rational functions and integrate up to some reference frequency, often chosen to be the time of coalescence, when the orbital frequency formally diverges. Thus, we obtain PN approximations of the form

$$
\begin{aligned}
& t(v)=t_{c}-\int_{v_{c}}^{v} \frac{E_{\mathrm{Newt}}}{F_{\mathrm{Newt}}}\left(2 v^{-9}+\sum_{j=2}^{7} t_{j} v^{j-9}\right), \\
& \Psi(v)=\Psi_{c}-\frac{1}{M} \int_{v_{c}}^{v} \frac{E_{\mathrm{Newt}}}{F_{\text {Newt }}}\left(2 v^{-6}+\sum_{j=2}^{7} t_{j} v^{j-6}\right),
\end{aligned}
$$


where the $t_{j}$ coefficients are linear combinations of products of the $E_{i}$ and $F_{i}$. Plugging Eqs. (6.20), (6.21) into Eq. (6.12), we obtain the following expression for the SPA phase through 2.5PN order ${ }^{10}$

$$
\begin{aligned}
\Psi_{\mathrm{SPA}}(F)= & 2 \pi F t_{c}-\Psi_{c}+\frac{3}{256}(2 \pi \mathcal{M} F)^{-5 / 3}\left\{1+\left(\frac{3715}{756}+\frac{55}{9} \nu\right)(2 \pi M F)^{2 / 3}+(4 \beta-16 \pi)(2 \pi M F)\right. \\
+ & \left(\frac{15293365}{508032}+\frac{27145}{504} \nu+\frac{3085}{72} \nu^{2}-10 \sigma\right)(2 \pi M F)^{4 / 3}+\left(\frac{38645}{756} \pi-\frac{65}{9} \pi \nu-\gamma\right)(1+3 \log (v)) \times \\
& \left.(2 \pi M F)^{5 / 3}\right\},
\end{aligned}
$$

where the 1.5PN SO phase corrections are contained in $\beta$, the 2PN SS corrections are contained in $\sigma$, and the 2.5PN SO corrections are contained in $\gamma$. Note that $\beta$ and the $\operatorname{spin}(1)-\operatorname{spin}(2)$ contributions to $\sigma$ were previously known [13, 14], while we have calculated the $\operatorname{spin}(1)-\operatorname{spin}(1)$ and $\operatorname{spin}(2)-\operatorname{spin}(2)$ contributions to $\sigma$ and the $2.5 \mathrm{PN}$ SO corrections to the SPA phase using the results for the center-of-mass energy and GW flux of Refs. [29, 30, 33, 75]. Explicitly, these corrections are

$$
\begin{aligned}
\beta & =\left(\frac{113}{12}-\frac{19}{3} \nu\right) \boldsymbol{\chi}_{s} \cdot \hat{\mathbf{L}}_{\mathrm{N}}+\frac{113}{12} \delta \boldsymbol{\chi}_{a} \cdot \hat{\mathbf{L}}_{\mathrm{N}} \\
\sigma & =\nu\left\{\frac{721}{48}\left[\left(\boldsymbol{\chi}_{s} \cdot \hat{\mathbf{L}}_{\mathrm{N}}\right)^{2}-\left(\boldsymbol{\chi}_{a} \cdot \hat{\mathbf{L}}_{\mathrm{N}}\right)^{2}\right]-\frac{247}{48}\left(\boldsymbol{\chi}_{s}^{2}-\boldsymbol{\chi}_{a}^{2}\right)\right\} \\
& +(1-2 \nu)\left\{\frac{719}{96}\left[\left(\boldsymbol{\chi}_{s} \cdot \hat{\mathbf{L}}_{\mathrm{N}}\right)^{2}+\left(\boldsymbol{\chi}_{a} \cdot \hat{\mathbf{L}}_{\mathrm{N}}\right)^{2}\right]-\frac{233}{96}\left(\boldsymbol{\chi}_{s}^{2}+\boldsymbol{\chi}_{a}^{2}\right)\right\} \\
& +\delta\left[\frac{719}{48}\left(\boldsymbol{\chi}_{s} \cdot \hat{\mathbf{L}}_{\mathrm{N}}\right)\left(\boldsymbol{\chi}_{a} \cdot \hat{\mathbf{L}}_{\mathrm{N}}\right)-\frac{233}{48} \boldsymbol{\chi}_{s} \cdot \boldsymbol{\chi}_{a}\right] \\
\gamma & =\left(\frac{732985}{2268}-\frac{24260}{81} \nu-\frac{340}{9} \nu^{2}\right) \boldsymbol{\chi}_{s} \cdot \widehat{\mathbf{L}}_{\mathrm{N}}+\left(\frac{732985}{2268}+\frac{140}{9} \nu\right) \delta \boldsymbol{\chi}_{a} \cdot \widehat{\mathbf{L}}_{\mathrm{N}} .
\end{aligned}
$$

We note that these expressions are only valid when both component spins are aligned or anti-aligned with the orbital angular momentum. The spin(1)-spin(1) and $\operatorname{spin}(2)-\operatorname{spin}(2)$ contributions to $\sigma$ were also derived in Refs. 32, 33. and we found full agreement with them.

\section{Features of frequency-domain non-precessing waveforms with higher harmonics}

We now discuss some interesting features of the spinning, non-precessing waveforms derived in Sec. VIB Several papers have studied the effect of higher harmonics in the amplitude corrections of non-spinning binaries observable by ground- and space-based detectors [47, 48, 52 [54, 88].

One important feature of the higher harmonics in the waveform amplitude is that they can increase the mass reach of a detector [52]. This is because high-mass binaries whose dominant second harmonic is not in the detector's sensitive band can have higher harmonics in band and therefore become visible to the detector. A closer look at Eq. (6.14) and Appendix $\mathrm{D}$ shows that spin corrections through 2PN order appear only in the first and second harmonics. In particular, the only SPA amplitude coefficients with spin dependence are $\mathcal{C}_{1}^{(2)}, \mathcal{C}_{2}^{(3)}$ and $\mathcal{C}_{2}^{(4)}$ given in Appendix D, Thus, in the non-precessing case spin corrections through $2 \mathrm{PN}$ order in the waveform amplitude do not affect the mass reach of the detector, and only affect binaries whose second harmonic appears in band.

Another general feature of the higher harmonics is that they interfere with one another, typically destructively [47, 52, 54]. For binaries that would be visible with Newtonian waveforms, this effect tends to decrease the signal to noise ratio (SNR). As we shall study in detail in this section, spin effects can play a role in this interference, either raising or lowering the SNR depending on the spin orientations.

We define the power spectrum, $P(f)$, as

$$
P(f)=\frac{|\tilde{h}(f)|^{2}}{S_{n}(f)}
$$

10 The non-spinning terms in the SPA phase through 3.5PN order can be found in Ref. [87]. 

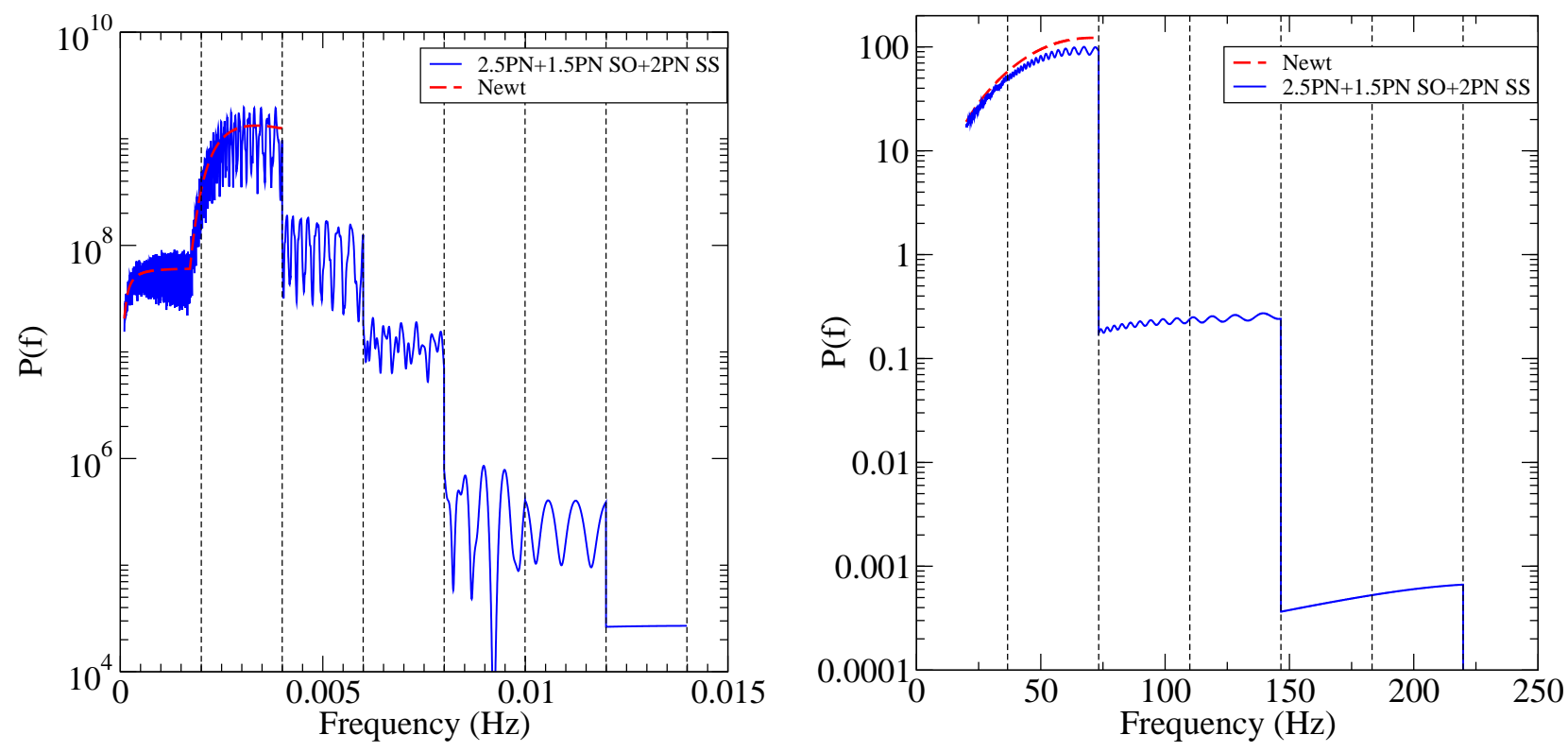

FIG. 5: We compare the power spectra computed with the Newtonian amplitude waveform (red dashed line) and the 2.5PN waveform with 1.5PN SO and 2PN SS effects included (blue, continuous line). In the left panel we consider a typical source for LISA, a binary with total mass $\left(10^{6}+10^{5}\right) M_{\odot}$, and spins maximal and aligned with the orbital angular momentum. In the right panel we consider a typical source for Advanced LIGO, a binary of total mass $(30+30) M_{\odot}$ with spins $\chi_{1}=1, \chi_{2}=0.5$ aligned with the orbital angular momentum. Note that the $k^{\text {th }}$ harmonic ends at $k F_{\mathrm{LSO}}$, and these frequencies are marked by the vertical dashed lines on the graph. The spectrum of the $2.5 \mathrm{PN}$ waveform is much simpler in the equal-mass case than unequal mass case because in the former case all non-spinning odd harmonics are suppressed.

and the optimal SNR, $\rho$, as

$$
\rho^{2}=4 \int_{f_{s}}^{7 F_{\mathrm{LSO}}} \frac{|\tilde{h}(f)|^{2}}{S_{n}(f)} d f
$$

where $f_{s}$ is the low frequency seismic cutoff of the detector, and the upper frequency cutoff is taken to be the highest harmonic of the orbital frequency at the last stable orbit (LSO) which for simplicity we choose to be the LSO of a test particle in Schwarzschild,

$$
F_{\mathrm{LSO}}=\frac{1}{2 \pi 6^{3 / 2} M}
$$

Note that the $k^{\text {th }}$ harmonic ends at $k F_{\mathrm{LSO}}$ as enforced by a step function $\theta\left(k F_{\mathrm{LSO}}-f\right)$ [see Eqs. (D1)-(D21) in Appendix D. In Eqs. (6.26), (6.27), we denote with $S_{n}(f)$ the noise power spectral density of the detector. For Advanced LIGO, we take the spectral density to be Eq. (4.3) of Ref. 48] and fix $f_{s}=20 \mathrm{~Hz}$. For LISA, we use the so-called effective non-sky-averaged spectral density given in Eqs. (2.28)-(2.32) of Ref. [89]. We do not consider the orbital motion of the LISA spacecraft [90] and consider only the single detector configuration ${ }^{11}$. In the presence of higher harmonics, the lower and upper cut-off frequencies are chosen following Sec. IIIA of Ref. [52]. For LISA we assume an observation time of one year, and the orbital frequency at the beginning of observation to be Eq. (3.3) of Ref. [52]. As explained in Ref. [52], this can be implemented by multiplying the $k^{\text {th }}$ harmonic by the step function $\theta\left(f-k F_{i n}\right)$ where $F_{i n}$ is the orbital frequency at the beginning of observation. Finally, because of the $60^{\circ}$ angle between LISA's arms, we use $\tilde{h}(f) \rightarrow(\sqrt{3} / 2) \tilde{h}(f)$ in Eqs. (6.26), (6.27) in the case of LISA.

All tables and figures in this section, refer to a binary with orbital angular momentum inclined relative to the line of sight by $\theta=\pi / 3$, sky location $\bar{\theta}=\bar{\phi}=\pi / 6$ and polarization angle $\bar{\psi}=\pi / 4$ [see Eqs. (2.4), (2.5)]. We have verified,

11 It should be noted that in our model, though we do not perform an average over the antenna pattern functions, we do not account for the orbital motion of LISA either. In this sense, our model falls in between the pattern averaged and non-pattern averaged cases described in Ref. [89] 


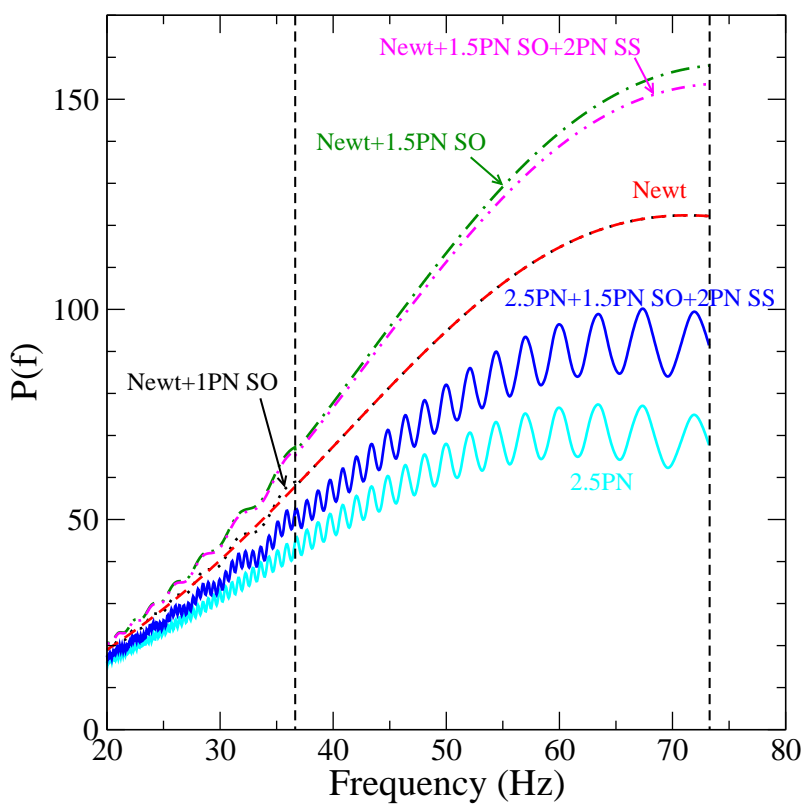

FIG. 6: For a binary of total mass $(30+30) M_{\odot}$ with spins $\chi_{1}=1, \chi_{2}=0.5$ aligned with the orbital angular momentum (the same binary of the right panel of Fig. (5), we show the power spectra up to $2 F_{\mathrm{LSO}}$. We plot the power spectrum for the waveform through 2.5 PN order with no spin corrections (cyan solid line) and with SO corrections through 1.5PN (that is, 1PN and $1.5 \mathrm{PN}$ ) and $\mathrm{SS}$ corrections at $2 \mathrm{PN}$ order (dark blue solid line). We also plot power spectra for the waveform with Newtonian amplitude (red dashed line), Newtonian amplitude plus the 1PN SO correction (black dotted line), Newtonian amplitude plus SO effects through 1.5PN (green,dot dashed line), and Newtonian amplitude plus SO corrections through $1.5 \mathrm{PN}$ and the $2 \mathrm{PN}$ SS correction (magenta, double-dot-dashed line). The 1.5PN SO and 2PN SS effects raise and lower the power in the dominant harmonic while the $1 \mathrm{PN}$ SO effect merely changes the modulation pattern up to its cutoff frequency of $F_{\mathrm{LSO}}$. Vertical dashed lines mark the frequencies $F_{\mathrm{LSO}}$ and $2 F_{\mathrm{LSO}}$.

by considering random values for the four angles, that the qualitative trends reported in this section are generic and do not depend on the specific values of them (see a detailed discussion at the end of this section). Regardless of the PN order of the amplitude, all waveforms use the SPA phase with non-spinning terms up to 3.5PN order [87], and spin terms up to 2.5PN order, as given in Eqs. (6.22) (6.25). In the case of Advanced LIGO (LISA) we consider binaries at a distance of $100 \mathrm{Mpc}(3 \mathrm{Gpc})$. Moreover, all masses and distances refer to the redshifted quantities.

The destructive interference between different harmonics can be seen in Fig. 5 . The Newtonian waveform's power spectrum is simply proportional to $f^{-7 / 3} / S_{n}(f)$. The higher harmonics present in the $2.5 \mathrm{PN}$ waveform introduce oscillatory cross terms that on average lower the power. Notice that although the higher harmonics extend the observable frequency band significantly, the power beyond the cutoff of the second harmonic, being at a higher PN order, is suppressed by one or several orders of magnitude. These features explain why the SNR listed in Tables [. III tends to decrease as the PN order increases for the range of masses we consider. For equal-mass binaries, all non-spinning odd harmonic corrections are suppressed because the latter are proportional to $\delta$ which is zero for equal masses [see Eqs. (D1)-(D21) in Appendix D. This is not true of spin-dependent amplitude corrections. For example, the first harmonic has a spin dependent amplitude correction at 1PN order which does not vanish for equal mass systems unless spins are equal and aligned with one another [see Eq. (6.6a)].

Tables [I. II show the SNR for the case of maximal spins both aligned or anti-aligned with the orbital angular momentum. From the bottom three rows of Tables $\mathrm{U}$. III we see that, depending on the spin orientation, the 2.5PN amplitude corrections with spins can have SNR 10\% higher or lower than the 2.5PN amplitude corrections without spins. We caution that this $\sim 10 \%$ change in the SNR from spin corrections is only meant as a bound on spin effects for spinning, non-precessing binaries. As we have seen in Sec. $\mathrm{V}$ the affect of spin corrections on precessing binaries is not bounded by the cases of maximal spins aligned and anti-aligned with the orbital angular momentum.

Quite interestingly, the 1.5PN SO and 2PN SS corrections are far more important than the 1PN SO correction in terms of their effect on the power spectrum and the SNR. Notice that in Tables I, I the 1PN SO term always has little or no effect, while the 1.5PN SO term changes the SNR by $\sim 10 \%$, and the 2PN SS term changes the SNR for the equal-mass binary. The reason the 1PN SO term is less important is that the 1.5PN SO and 2PN SS terms are corrections to the second harmonic, so they increase or decrease the power in the dominant term. On the other hand, 


\begin{tabular}{|l|c|c|c|c|}
\hline & \multicolumn{3}{|c|}{ Advanced LIGO SNR } \\
& $(50+5) M_{\odot}$ & $(30+30) M_{\odot}$ \\
& $(1,1)$ & $(-1,-1)$ & $(1,1)$ & $(-1,-1)$ \\
\hline Newt & 76.4 & 76.4 & 131.1 & 131.1 \\
$0.5 \mathrm{PN}$ & 84.9 & 82.3 & 131.1 & 131.1 \\
$1 \mathrm{PN}$ & 74.2 & 71.9 & 116.9 & 115.8 \\
$1 \mathrm{PN}+1 \mathrm{PN} \mathrm{SO}$ & 74.1 & 72.1 & 116.9 & 115.8 \\
$1.5 \mathrm{PN}$ & 69.2 & 67.6 & 116.9 & 115.8 \\
$1.5 \mathrm{PN}+1.5 \mathrm{PN} S O$ & 79.7 & 58.1 & 134.2 & 98.8 \\
$2 \mathrm{PN}+1.5 \mathrm{PN} S O$ & 75.8 & 55.1 & 123.1 & 88.3 \\
$2 \mathrm{PN}+1.5 \mathrm{PN} \mathrm{SO}+2 \mathrm{PN} \mathrm{SS}$ & 75.5 & 54.8 & 121.4 & 86.7 \\
$2.5 \mathrm{PN}$ & 64.0 & 62.6 & 106.3 & 105.3 \\
$2.5 \mathrm{PN}+1.5 \mathrm{PN} S \mathrm{SO}$ & 74.2 & 53.6 & 123.5 & 88.5 \\
$2.5 \mathrm{PN}+1.5 \mathrm{PN} \mathrm{SO}+2 \mathrm{PN} \mathrm{SS}$ & 73.9 & 53.4 & 121.7 & 86.9 \\
\hline
\end{tabular}

TABLE I: For several binary configurations observable by Advanced LIGO we list the SNR as the PN order of the amplitude corrections is varied. In each column we show the component spins $\left(\chi_{1} \cdot \hat{\mathbf{L}}_{\mathrm{N}}, \chi_{2} \cdot \hat{\mathbf{L}}_{\mathrm{N}}\right)$. We include all non-spinning, spin-orbit $(\mathrm{SO})$ and spin-spin $(\mathrm{SS})$ corrections up to the orders given in the first column. For example, $2.5 \mathrm{PN}+1.5 \mathrm{PN} \mathrm{SO}+2 \mathrm{PN} \mathrm{SS}$ means we include non-spinning amplitude corrections from Newtonian to 2.5PN order, $1 \mathrm{PN}$ and $1.5 \mathrm{PN} \mathrm{SO}$ corrections, and the 2PN SS correction. Regardless of the PN order of the amplitude, we always use the SPA phase with non-spinning terms up to 3.5PN order, and spin terms up to 2.5PN order, as given in Eqs. (6.22) - 6.25). The binary is at a distance of $100 \mathrm{Mpc}$ with orbital angular momentum inclined relative to the line of sight by $\theta=\pi / 3$, sky location $\bar{\theta}=\bar{\phi}=\pi / 6$ and polarization angle $\bar{\psi}=\pi / 4$ [see Eqs. (2.4), (2.5)].

\begin{tabular}{|c|c|c|c|c|}
\hline & $\begin{array}{l}(2 \times 10 \\
(1,1)\end{array}$ & $\begin{array}{r}\text { LISA } \\
\left.+10^{4}\right) M_{\odot} \\
(-1,-1)\end{array}$ & $\begin{array}{l}\text { SR } \\
\left(10^{6}+\right. \\
(1,1)\end{array}$ & $\begin{array}{l}\left.10^{6}\right) M_{\odot} \\
(-1,-1)\end{array}$ \\
\hline Newt & 382.6 & 382.6 & 2764.4 & 2764.4 \\
\hline $0.5 \mathrm{PN}$ & 598.5 & 598.6 & 2764.4 & 2764.4 \\
\hline $1 \mathrm{PN}$ & 620.1 & 621.5 & 2510.0 & 2469.7 \\
\hline $1 \mathrm{PN}+1 \mathrm{PN} \mathrm{SO}$ & 619.9 & 621.7 & 2510.0 & 2469.7 \\
\hline $1.5 \mathrm{PN}$ & 512.2 & 517.0 & 2510.0 & 2469.7 \\
\hline $1.5 \mathrm{PN}+1.5 \mathrm{PN} \mathrm{SO}$ & 551.9 & 484.1 & 2875.9 & 2118.2 \\
\hline $2 \mathrm{PN}+1.5 \mathrm{PN} \mathrm{SO}$ & 523.6 & 457.5 & 2608.5 & 1870.5 \\
\hline $2 \mathrm{PN}+1.5 \mathrm{PN} \mathrm{SO}+2 \mathrm{PN} \mathrm{SS}$ & 523.5 & 457.4 & 2570.2 & 1836.1 \\
\hline $2.5 \mathrm{PN}$ & 479.4 & 481.1 & 2280.3 & 2242.0 \\
\hline $2.5 \mathrm{PN}+1.5 \mathrm{PN} \mathrm{SO}$ & 516.5 & 451.6 & 2639.2 & 1901.9 \\
\hline $2.5 \mathrm{PN}+1.5 \mathrm{PN} \mathrm{SO}+2 \mathrm{PN} \mathrm{SS}$ & 516.4 & 451.6 & 2601.4 & 1868.4 \\
\hline
\end{tabular}

TABLE II: For several binary configurations observable by LISA we list the SNR as the PN order of the amplitude corrections is varied. In each column we show the component spins $\left(\boldsymbol{\chi}_{1} \cdot \hat{\mathbf{L}}_{\mathrm{N}}, \boldsymbol{\chi}_{2} \cdot \hat{\mathbf{L}}_{\mathrm{N}}\right)$. We include all non-spinning, spin-orbit (SO) and spin-spin (SS) corrections up to the orders given in the first column. Regardless of the PN order of the amplitude, we always use the SPA phase with non-spinning terms up to 3.5PN order, and spin terms up to 2.5PN order, as given in Eqs. 66.22)-66.25). The binary is at a distance of $3 \mathrm{Gpc}$ with the same orientation as in Table I The binary masses and distances refer to the redshifted quantities.

the $1 \mathrm{PN}$ SO term is a correction to the first harmonic. Thus, it is merely a perturbation to the dominant signal, and only in the lowest part of the spectrum where the first harmonic is observable. This is illustrated in Fig. 6, where we plot the power spectrum as a function of frequency (up to $2 F_{\mathrm{LSO}}$ ) for different spin contributions for the $(30+30) M_{\odot}$ binary system. We see that the 1.5PN SO and 2PN SS corrections add or subtract their power coherently with the dominant second harmonic. Their net effect is to shift the power spectrum of the full waveform upward without changing its shape. On the other hand, the 1PN SO correction, which is proportional to the (sine or cosine of) half the dominant harmonic, simply changes the modulation pattern of the full waveform up to $F_{\mathrm{LSO}}(37 \mathrm{~Hz}$ ). It should however be noted that the structures in the power spectra could be more complicated for asymmetric systems where the non-spinning terms proportional to $\cos \Psi$ and $\sin \Psi$ are not suppressed.

The 1.5PN SO term is typically the most important of the spin terms. This term is linearly proportional to the spins of the two bodies, as can be seen in Eq. (D8). If the spins are aligned with the orbital angular momentum it increases the SNR. If the spin terms are anti-aligned with the orbital angular momentum it decreases the SNR. If one spin is aligned with $\hat{\mathbf{L}}_{\mathrm{N}}$ and the other anti-aligned, the body with the greater spin $\mathbf{S}_{i}=m_{i}^{2} \chi_{i}$, which is typically the 


\begin{tabular}{|l|c|c|c|c|}
\hline & \multicolumn{4}{|c|}{$\begin{array}{c}\text { Advanced LIGO SNR } \\
(60+40) M_{\odot}\end{array}$} \\
& $(1,-1)$ & $(0.8,-0.8)$ & $(0.5,-0.5)$ & $(0.2,-0.2)$ \\
& & & & 81.8 \\
$2.5 \mathrm{PN}$ & 81.0 & 80.5 & 80.8 & 82.5 \\
$2.5 \mathrm{PN}+1.5 \mathrm{PN}$ SO & 84.4 & 83.3 & 82.5 & 82.5 \\
$2.5 \mathrm{PN}+1.5 \mathrm{PN} \mathrm{SO}+2 \mathrm{PN} \mathrm{SS}$ & 85.8 & 84.2 & 82.8 & 8.2 \\
\hline
\end{tabular}

TABLE III: For a typical binary observable by Advanced LIGO, we compare the SNR obtained using the 2.5PN amplitude corrected waveform without spin effects, with spin-orbit effects, and with spin-orbit and spin-spin effects. In each column we show the component spins $\left(\boldsymbol{\chi}_{1} \cdot \hat{\mathbf{L}}_{\mathrm{N}}, \chi_{2} \cdot \hat{\mathbf{L}}_{\mathrm{N}}\right)$. In all cases we use the SPA phase with non-spinning terms up to $3.5 \mathrm{PN}$ order, and spin terms up to 2.5PN order, as given in Eqs. 6.22) - 6.25). The binary is at a distance of 100 Mpc with the same orientation as in Table \

larger body, dominates. Thus, the large body dictates whether the SO effect increases or decreases the SNR, unless the spin of the smaller body is much greater than the spin of the large body. This is illustrated in Table IV] where the mass ratio $m_{1}: m_{2}=10: 1$. The spin of the larger body is aligned with $\hat{\mathbf{L}}_{\mathrm{N}}$ and tends to increase the SNR while the spin of the smaller body is anti-aligned with $\hat{\mathbf{L}}_{\mathrm{N}}$ and tends to decrease the SNR. For a spin ratio $\chi_{1}: \chi_{2}=1: 1$ there is a large increase in SNR due the larger BH. For a spin ratio 1:10, the larger BH still dominates and we get a small increase in SNR. For the spin ratios of 1:100 and 1:1000, the smaller BH is now able to overcome the larger $\mathrm{BH}$ and produce a net decrease in the SNR.

\begin{tabular}{|c|c|c|c|c|}
\hline & $(1,-1)$ & $\begin{array}{r}\mathrm{LI} \\
\left(10^{6}\right. \\
(0.1,-1)\end{array}$ & $\begin{array}{l}\text { A SNR } \\
\left.+10^{5}\right) M_{\odot} \\
(0.01,-1)\end{array}$ & $(0.001,-1)$ \\
\hline $2.5 \mathrm{PI}$ & 2538.7 & 2570.4 & 2522.2 & 2572.4 \\
\hline $2.5 \mathrm{PN}+1.5 \mathrm{PN} \mathrm{SO}$ & 2917.5 & 2583.8 & 2500.6 & 2546.7 \\
\hline $2.5 \mathrm{PN}+1.5 \mathrm{PN} \mathrm{SO}+2 \mathrm{PN} \mathrm{SS}$ & 2930.4 & 2585.0 & 2500.8 & 2546.7 \\
\hline
\end{tabular}

TABLE IV: For a typical binary observable by LISA, we compare the SNR obtained using the $2.5 \mathrm{PN}$ waveform without spin effects, with spin-orbit effects, and with spin-orbit and spin-spin effects. In each column we show the component spins $\left(\chi_{1} \cdot \hat{\mathbf{L}}_{\mathrm{N}}, \chi_{2} \cdot \hat{\mathbf{L}}_{\mathrm{N}}\right)$. In all cases we use the SPA phase with non-spinning terms up to 3.5PN order, and spin terms up to $2.5 \mathrm{PN}$ order, as given in Eqs. (6.22) - 6.25). The binary is at a distance of 3 Gpc with the same orientation as in Table I The binary masses and distances refer to the redshifted quantities.

The 2PN SS term decreases the power spectrum and SNR when the component spins are aligned with one another, and increases the power spectrum and SNR when they are anti-aligned with one another. The 2PN SS term has a greater effect on the SNR and power spectrum than the 1PN SO term, but is less important than the 1.5PN SO term. This is because it is suppressed relative to the 1.5PN SO term by a factor of $v / c$ and it is quadratic in the spins and proportional to the symmetric mass-ratio $\nu$. Thus, the 2PN SS term are most important for binaries with two large component spins and comparable masses. From Tables 【, III, we can see that the 2PN SS term has little or no effect on binaries with a mass ratio greater than 10:1. In Tables I II for the columns with equal masses and spins aligned with one another, the 2PN SS term decreases the SNR by a few percent. For the binary in Table III we see that the 2PN SS term increases the SNR by an amount comparable to the SO terms when the spins are maximal. As we decrease the spin magnitude, the SS effect is suppressed faster than the SO effect because it is quadratic in the spins while the SO effect is linear.

Before ending this section we study how different values of the source position and inclination angle can affect the SNR trends shown in Table I. For $(5+50) M_{\odot}$ and $(30+30) M_{\odot}$ systems we calculated the SNRs at different PN orders in amplitude for various random realizations of $\bar{\theta}, \bar{\phi}, \bar{\psi}$ and $\theta$ and for the spinning and non-spinning cases. For the spinning cases, when all the known spin effects are included at different PN orders, the trends across different orders remains the same for all the random realizations except between the Newtonian and 0.5PN order. Though on most of the occasions, the SNR increases from Newtonian to 0.5PN order, there are cases when it decreases, albeit slightly. All these cases where the SNR decreases have inclination angle $\theta$ very close to zero or $\pi$. For these cases, the third harmonic, which is proportional to $\sin \theta$, is largely suppressed and the spin-dependent interference accounts for the small drop in SNR. This drop is observed for systems for which $\chi_{1}=\chi_{2}=-1$ whereas the non-spinning and $\chi_{1}=\chi_{2}=1$ cases consistently showed the increase in SNR between Newtonian and 0.5PN order. To further assert this, we fix the inclination angle to a value very close to zero and $\pi$ and randomly varied the other three angles. We find that for all the realizations the SNR decreases in going from Newtonian to $0.5 \mathrm{PN}$ order. In brief, the trends shown in Table $\square$ is quite general except for inclination angles close to zero or $\pi$. We however note that the trends of 
Table \need not be same for much higher masses when the leading harmonic approaches the lower cut-off frequency of the detector $\left(2 F_{\mathrm{LSO}} \simeq f_{s}\right)$. We have not done a thorough analysis for the whole mass range.

\section{CONCLUSIONS}

The ongoing search for GWs from compact binaries with the network of interferometers LIGO, Virgo and GEO, and the work at the interface between analytical and numerical relativity aimed at providing accurate templates for the search, has made it urgent to include higher-order PN effects in the theoretical predictions of the waveforms. This paper is a step forward in this direction.

We provided ready-to-use time-domain waveforms for spinning, precessing binaries moving on nearly circular orbits through 1.5PN order and decompose those waveforms in spin-weighted -2 spherical harmonics [see Appendices $\mathrm{A}$ and B. Neglecting radiation-reaction effects and assuming $S \ll L$, we found that the inclination angle $\iota$ between the total angular momentum and the Newtonian orbital angular momentum (see Fig. (1) is a 0.5PN correction. Motivated by this, we expanded the GW polarizations and spin-weighted spherical harmonic modes in a Taylor series in $\iota$ [see Eqs. (3.16), (3.17) and Eqs. (4.17a)-(4.17r)]. Their expressions become much simpler and allow one to extract interesting physical features of the gravitational waves from precessing binaries.

We found that, in contrast to what happens in the non-spinning case, the $h_{\ell m}$ 's are not in general proportional to $e^{-i m \Psi}$. They also depend on the angles $\iota$ and $\alpha$, where $\iota$ is the inclination angle of the Newtonian orbital momentum relative to the total angular momentum and $\alpha$ is the angle between the $x$-axis and the projection of the Newtonian orbital angular momentum onto the $x-y$ plane (see Fig. 1). For example, the terms independent of $\iota$ are proportional to $e^{-i m(\Psi+\alpha)}$, the terms that are linear in $\iota$ are proportional to $e^{-i\left(m+m^{\prime}\right)(\Psi+\alpha)} \cos \Psi$ or $e^{-i\left(m+m^{\prime}\right)(\Psi+\alpha)} \sin \Psi$, while higher-order contributions in $\iota$ involve terms of the form $e^{-i\left(m+m^{\prime}\right)(\Psi+\alpha)} \cos ^{a} \Psi \sin ^{b} \Psi$, where $a, b \in \mathbb{N}$ and $m^{\prime} \in$ $-1,0,1$. In the presence of precession, the angles $\iota$ and $\alpha$ vary in time and the different harmonics present in each of the modes interfere, causing a strong modulation of the mode amplitudes. We also found that, in contrast to what happens in the non-spinning case, the signal can be largely distributed among modes $(\ell, m)$ other than the $(2,2)$ mode. With our choice of the source frame, when spins are maximal and the binary system has significant mass asymmetry and/or a large inclination angle, we found that the amplitude of the $(2,0)$ and $(2,1)$ modes can be comparable to the amplitude of the $(2,2)$ mode, especially during the last stages of inspiral. For the mass ratios we considered, we found that the $\ell=3$ and $\ell=4$ modes are generally one or two orders of magnitude smaller than the $\ell=2$ modes. These results are summarized in Figs. 2, 3] and 4 for binaries with mass ratio 1:1 and 4:1, and for two maximal spin configurations having a small or large inclination angle $\iota$. The ready-to-use time-domain waveforms for spinning, precessing binaries can be employed for accurate comparisons with numerical simulations of binary BHs [66, 80, 91 95] and for designing time-domain [63, 67, 69, 70] analytical templates.

Restricting ourselves to spinning, non-precessing binaries, we computed ready-to-use frequency-domain waveforms in the stationary-phase approximation. We derived $1 \mathrm{PN}$ and $1.5 \mathrm{PN}$ order spin-orbit effects, and $2 \mathrm{PN}$ order spinspin (spin(1)-spin(2) only) effects in the frequency-domain GW amplitude [see Eq. (6.14), and Eqs.(D1)-(D21) in Appendix $\mathrm{D}$. We also calculated the $2 \mathrm{PN}$ spin-spin (including spin(1)-spin(1) and $\operatorname{spin}(2)$-spin(2) effects), and the 2.5PN order spin-orbit effects in the frequency-domain GW phase [see Eqs. (6.22), (6.25) and (6.24)]. For the 2PN spin-spin terms, we found agreement with Refs. 32, 33]. We wrote the frequency-domain waveforms in a rather compact way, so that they can be easily used for data analysis and for building analytical frequency-domain 72, 73. templates.

In the non-precessing case, we found that, through $2 \mathrm{PN}$ order, spin effects in the amplitude affect only the PN corrections to the first and second harmonics. Thus, through 2PN order, spin effects do not yet extend the mass reach of GW detectors. However, as seen in Figs. 5] 6. they can interfere with other harmonics and, depending on the spin orientation, lower or raise the signal-to-noise ratio of ground-based (see Tables II III) and space-based detectors (see Tables II] IV). We also expect that those spin terms will help in localizing the binary source in the sky. We leave to a future publication the use of the waveforms derived in this paper to extend parameter-estimation predictions [15, 45 58, 87, 89] of ground-based and space-based detectors to spinning, precessing binaries.

Finally, we notice that the gravitational polarizations computed in this paper do not include the modification of the orbital phase evolution at the relative 2.5PN order induced by the flow of energy into the black hole horizons as explicitly computed in Ref. [96]. As summarized in Table IV of Ref. [96], this effect can cause a variation of the number of GW cycles at the Schwarzschild ISCO of 3\%-24\% depending on the binary mass ratio. We postpone to a future publication the inclusion of those effects. 


\section{Acknowledgments}

We thank Luc Blanchet, Bala Iyer, Yi Pan and Etienne Racine for useful discussions. K.G.A is a VESF fellow of the European Gravitational Observatory (EGO). A.B. and E.O. acknowledge support from NSF grant PHY-0603762, and A.B. also acknowledges support from the Alfred P Sloan Foundation.

\section{Appendix A: Ready-to-use gravitational-wave polarizations for precessing binaries on nearly circular orbits through 1.5PN order: generic inclination angles}

In Sec. III we wrote the GW polarizations

$$
h_{+, \times}=\frac{2 M \nu v^{2}}{D_{L}}\left[\hat{h}_{+, \times}^{(0)}+\left(\hat{h}_{+, \times}^{(1 / 2)}+\hat{h}_{+, \times}^{(1 / 2, \mathrm{SO})}\right) v+\left(\hat{h}_{+, \times}^{(1)}+\hat{h}_{+, \times}^{(1, \mathrm{SO})}\right) v^{2}+\left(\hat{h}_{+, \times}^{(3 / 2)}+\hat{h}_{+, \times}^{(3 / 2, \mathrm{SO})}\right) v^{3}\right]
$$

expanded in the inclination angle $\iota$. Here we give the full expressions. The Newtonian, 0.5PN and 1PN order coefficients were computed explicitly in Ref. 34], the 1.5PN order coefficients, are computed for the first time in this paper. They read

$$
\begin{aligned}
& \hat{h}_{+}^{(0)}=\left(-\frac{3}{2}-\frac{c_{2 \theta}}{2}\right) c_{\frac{\iota}{2}}^{4} \cos (2 \alpha+2 \Psi)-2 c_{\frac{\iota}{2}}^{3} \cos (\alpha+2 \Psi) s_{2 \theta} s_{\frac{\iota}{2}}+2 c_{\frac{\iota}{2}} \cos (\alpha-2 \Psi) s_{2 \theta} s_{\frac{\iota}{2}}^{3} \\
& +\left(-\frac{3}{2}-\frac{c_{2 \theta}}{2}\right) \cos (2 \alpha-2 \Psi) s_{\frac{\iota}{2}}^{4}-\frac{3}{2} \cos (2 \Psi) s_{\theta}^{2} s_{\iota}^{2}, \\
& \hat{h}_{+}^{(1 / 2)}=\delta\left[c_{\frac{\iota}{2}}^{6} \cos (3 \alpha+3 \Psi)\left(-\frac{45 s_{\theta}}{32}-\frac{9 s_{3 \theta}}{32}\right)+c_{\frac{\iota}{2}}^{2} \cos (\alpha+\Psi)\left(-\frac{175 s_{\theta}}{256}+c_{\iota}\left(\frac{87 s_{\theta}}{64}-\frac{5 s_{3 \theta}}{64}\right)\right.\right. \\
& \left.+c_{2 \iota}\left(-\frac{5 s_{\theta}}{256}+\frac{15 s_{3 \theta}}{256}\right)+\frac{13 s_{3 \theta}}{256}\right)+\cos (\alpha-\Psi)\left(\frac{175 s_{\theta}}{256}+c_{\iota}\left(\frac{87 s_{\theta}}{64}-\frac{5 s_{3 \theta}}{64}\right)\right. \\
& \left.+c_{2 \iota}\left(\frac{5 s_{\theta}}{256}-\frac{15 s_{3 \theta}}{256}\right)-\frac{13 s_{3 \theta}}{256}\right) s_{\frac{\iota}{2}}^{2}+c_{\frac{\iota}{2}}^{4} \cos (3 \alpha+\Psi)\left(-\frac{5 s_{\theta}}{32}-\frac{s_{3 \theta}}{32}\right) s_{\frac{\iota}{2}}^{2} \\
& +c_{\frac{\iota}{2}}^{4} \cos (\alpha+3 \Psi)\left(-\frac{45 s_{\theta}}{32}+\frac{135 s_{3 \theta}}{32}\right) s_{\frac{\iota}{2}}^{2}+c_{\frac{\iota}{2}}^{2} \cos (\alpha-3 \Psi)\left(\frac{45 s_{\theta}}{32}-\frac{135 s_{3 \theta}}{32}\right) s_{\frac{\iota}{2}}^{4} \\
& +c_{\frac{\iota}{2}}^{2} \cos (3 \alpha-\Psi)\left(\frac{5 s_{\theta}}{32}+\frac{s_{3 \theta}}{32}\right) s_{\frac{\iota}{2}}^{4}+\left(\frac{27}{16}+\frac{9 c_{2 \theta}}{16}\right) \cos (3 \alpha-3 \Psi) s_{\theta} s_{\frac{\iota}{2}}^{6}+\frac{45}{16} c_{\theta} \cos (3 \Psi) s_{\theta}^{2} s_{\iota}^{3} \\
& +\cos (2 \alpha+\Psi)\left(\left(-\frac{85 c_{\theta}}{256}-\frac{1}{128} c_{\theta} c_{2 \theta}-\frac{1}{32} c_{\theta} c_{2 \theta} c_{\iota}-\frac{3}{128} c_{\theta} c_{2 \theta} c_{2 \iota}\right) s_{\iota}-\frac{11}{64} c_{\theta} s_{2 \iota}-\frac{1}{256} c_{\theta} s_{3 \iota}\right) \\
& +\cos (2 \alpha+3 \Psi)\left(\left(\frac{45 c_{\theta}}{256}+\frac{81}{128} c_{\theta} c_{2 \theta}+\frac{27}{32} c_{\theta} c_{2 \theta} c_{\iota}+\frac{27}{128} c_{\theta} c_{2 \theta} c_{2 \iota}\right) s_{\iota}+\frac{9}{64} c_{\theta} s_{2 \iota}+\frac{9}{256} c_{\theta} s_{3 \iota}\right) \\
& +\cos (2 \alpha-\Psi)\left(\left(-\frac{85 c_{\theta}}{256}+\frac{1}{256} c_{\theta} c_{2 \theta}\right) s_{\iota}+\left(\frac{11 c_{\theta}}{64}+\frac{1}{64} c_{\theta} c_{2 \theta}\right) s_{2 \iota}+\left(-\frac{c_{\theta}}{256}-\frac{3}{256} c_{\theta} c_{2 \theta}\right) s_{3 \iota}\right) \\
& +\cos (2 \alpha-3 \Psi)\left(\left(\frac{45 c_{\theta}}{256}+\frac{135}{256} c_{\theta} c_{2 \theta}\right) s_{\iota}+\left(-\frac{9 c_{\theta}}{64}-\frac{27}{64} c_{\theta} c_{2 \theta}\right) s_{2 \iota}+\left(\frac{9 c_{\theta}}{256}+\frac{27}{256} c_{\theta} c_{2 \theta}\right) s_{3 \iota}\right) \\
& \left.+\cos (\Psi)\left(\frac{1}{64} c_{\theta} s_{\theta}^{2} s_{\iota}+\frac{5}{64} c_{\theta} s_{\theta}^{2} s_{3 \iota}\right)\right] \\
& \hat{h}_{+}^{(1)}=\left[c_{\frac{\iota}{2}}^{4}\left(\frac{59}{16}+\frac{5 c_{2 \theta}}{2}-\frac{3 c_{4 \theta}}{16}+\left(\frac{5}{24}-\frac{11 c_{2 \theta}}{6}+\frac{7 c_{4 \theta}}{24}\right) c_{\iota}+\left(-\frac{5}{48}-\frac{c_{2 \theta}}{12}-\frac{7 c_{4 \theta}}{48}\right) c_{2 \iota}\right)\right. \\
& \left.+\nu c_{\frac{\iota}{2}}^{4}\left(-\frac{25}{16}-\frac{13 c_{2 \theta}}{3}+\frac{9 c_{4 \theta}}{16}+\left(-\frac{5}{8}+\frac{11 c_{2 \theta}}{2}-\frac{7 c_{4 \theta}}{8}\right) c_{\iota}+\left(\frac{5}{16}+\frac{c_{2 \theta}}{4}+\frac{7 c_{4 \theta}}{16}\right) c_{2 \iota}\right)\right] \cos (2 \alpha+2 \Psi) \\
& +\cos (4 \alpha+4 \Psi)\left(\left(-2-\frac{2 c_{2 \theta}}{3}\right) c_{\frac{\iota}{2}}^{8} s_{\theta}^{2}+\nu\left(6+2 c_{2 \theta}\right) c_{\frac{\iota}{2}}^{8} s_{\theta}^{2}\right)+\cos (3 \alpha+4 \Psi)\left(\frac{32}{3} c_{\theta}^{3} c_{\frac{L}{2}}^{7} s_{\theta} s_{\frac{\iota}{2}}-32 \nu c_{\theta}^{3} c_{\frac{\iota}{2}}^{7} s_{\theta} s_{\frac{\iota}{2}}\right) \\
& +\cos (3 \alpha+2 \Psi)\left[c_{\frac{\iota}{2}}^{5}\left(\left(-\frac{5}{6}+\frac{c_{2 \theta}}{6}\right) s_{2 \theta}-\frac{2}{3} c_{\theta}^{2} c_{\iota} s_{2 \theta}\right) s_{\frac{\iota}{2}}+\nu c_{\frac{\iota}{2}}^{5}\left(\left(\frac{5}{2}-\frac{c_{2 \theta}}{2}\right) s_{2 \theta}+2 c_{\theta}^{2} c_{\iota} s_{2 \theta}\right) s_{\frac{\iota}{2}}\right] \\
& +\cos (2 \alpha+4 \Psi)\left(\left(-\frac{10}{3}-\frac{8 c_{2 \theta}}{3}-\frac{14 c_{4 \theta}}{3}\right) c_{\frac{\iota}{2}}^{6} s_{\frac{\iota}{2}}^{2}+\nu\left(10+8 c_{2 \theta}+14 c_{4 \theta}\right) c_{\frac{\iota}{2}}^{6} s_{\frac{\iota}{2}}^{2}\right)
\end{aligned}
$$




$$
\begin{aligned}
& +\cos (4 \alpha+2 \Psi)\left(\left(-\frac{1}{2}-\frac{c_{2 \theta}}{6}\right) c_{\frac{\iota}{2}}^{6} s_{\theta}^{2} s_{\frac{\iota}{2}}^{2}+\nu\left(\frac{3}{2}+\frac{c_{2 \theta}}{2}\right) c_{\frac{\iota}{2}}^{6} s_{\theta}^{2} s_{\frac{\iota}{2}}^{2}\right)+\cos (\alpha+4 \Psi)\left(\left(\frac{8}{3}-\frac{56 c_{2 \theta}}{3}\right) c_{\frac{\iota}{2}}^{5} s_{2 \theta} s_{\frac{\iota}{2}}^{3}\right. \\
& \left.+\nu\left(-8+56 c_{2 \theta}\right) c_{\frac{\iota}{2}}^{5} s_{2 \theta} s_{\frac{\iota}{2}}^{3}\right)+\cos (\alpha-2 \Psi)\left[\nu \left(c_{\frac{\iota}{2}}\left(\frac{16 s_{2 \theta}}{3}+\frac{31}{4} c_{\iota} s_{2 \theta}+\frac{1}{4} c_{2 \iota} s_{2 \theta}-\frac{19 s_{4 \theta}}{16}\right)-\frac{7}{8} c_{\frac{3 \iota}{2}} s_{4 \theta}\right.\right. \\
& \left.\left.-\frac{7}{16} c_{\frac{5 \iota}{2}} s_{4 \theta}\right) s_{\frac{\iota}{2}}^{3}+\left(c_{\frac{\iota}{2}}\left(-6 s_{2 \theta}-\frac{31}{12} c_{\iota} s_{2 \theta}-\frac{1}{12} c_{2 \iota} s_{2 \theta}+\frac{19 s_{4 \theta}}{48}\right)+\frac{7}{24} c_{\frac{3 \iota}{2}} s_{4 \theta}+\frac{7}{48} c_{\frac{5 \iota}{2}} s_{4 \theta}\right) s_{\frac{\iota}{2}}^{3}\right] \\
& +\cos (2 \alpha-2 \Psi)\left[\left(\frac{59}{16}+\frac{5 c_{2 \theta}}{2}-\frac{3 c_{4 \theta}}{16}+\left(-\frac{5}{24}+\frac{11 c_{2 \theta}}{6}-\frac{7 c_{4 \theta}}{24}\right) c_{\iota}+\left(-\frac{5}{48}-\frac{c_{2 \theta}}{12}-\frac{7 c_{4 \theta}}{48}\right) c_{2 \iota}\right) s_{\frac{\iota}{2}}^{4}\right. \\
& \left.+\nu\left(-\frac{25}{16}-\frac{13 c_{2 \theta}}{3}+\frac{9 c_{4 \theta}}{16}+\left(\frac{5}{8}-\frac{11 c_{2 \theta}}{2}+\frac{7 c_{4 \theta}}{8}\right) c_{\iota}+\left(\frac{5}{16}+\frac{c_{2 \theta}}{4}+\frac{7 c_{4 \theta}}{16}\right) c_{2 \iota}\right) s_{\frac{\iota}{2}}^{4}\right] \\
& +\cos (\alpha-4 \Psi)\left(\nu\left(8-56 c_{2 \theta}\right) c_{\frac{\iota}{2}}^{3} s_{2 \theta} s_{\frac{\iota}{2}}^{5}+\left(-\frac{8}{3}+\frac{56 c_{2 \theta}}{3}\right) c_{\frac{\iota}{2}}^{3} s_{2 \theta} s_{\frac{\iota}{2}}^{5}\right)+\cos (3 \alpha-2 \Psi)\left[c _ { \frac { \iota } { 2 } } \left(\left(\frac{5}{6}-\frac{c_{2 \theta}}{6}\right) s_{2 \theta}\right.\right. \\
& \left.\left.-\frac{2}{3} c_{\theta}^{2} c_{\iota} s_{2 \theta}\right) s_{\frac{\iota}{2}}^{5}+\nu c_{\frac{\iota}{2}}\left(\left(-\frac{5}{2}+\frac{c_{2 \theta}}{2}\right) s_{2 \theta}+2 c_{\theta}^{2} c_{\iota} s_{2 \theta}\right) s_{\frac{L}{2}}^{5}\right]+\cos (2 \alpha-4 \Psi)\left(\left(-\frac{10}{3}-\frac{8 c_{2 \theta}}{3}-\frac{14 c_{4 \theta}}{3}\right) c_{\frac{\iota}{2}}^{2} s_{\frac{L}{2}}^{6}\right. \\
& \left.+\nu\left(10+8 c_{2 \theta}+14 c_{4 \theta}\right) c_{\frac{\iota}{2}}^{2} s_{\frac{L}{2}}^{6}\right)+\cos (4 \alpha-2 \Psi)\left(\left(-\frac{1}{2}-\frac{c_{2 \theta}}{6}\right) c_{\frac{\iota}{2}}^{2} s_{\theta}^{2} s_{\frac{L}{2}}^{6}+\nu\left(\frac{3}{2}+\frac{c_{2 \theta}}{2}\right) c_{\frac{L}{2}}^{2} s_{\theta}^{2} s_{\frac{L}{2}}^{6}\right) \\
& +\cos (3 \alpha-4 \Psi)\left(-\frac{32}{3} c_{\theta}^{3} c_{\frac{\iota}{2}} s_{\theta} s_{\frac{\iota}{2}}^{7}+32 \nu c_{\theta}^{3} c_{\frac{\iota}{2}} s_{\theta} s_{\frac{\iota}{2}}^{7}\right)+\cos (4 \alpha-4 \Psi)\left(\left(-2-\frac{2 c_{2 \theta}}{3}\right) s_{\theta}^{2} s_{\frac{\iota}{2}}^{8}+\nu\left(6+2 c_{2 \theta}\right) s_{\theta}^{2} s_{\frac{\iota}{2}}^{8}\right) \\
& +\cos (2 \Psi)\left[\left(\left(\frac{349}{96}-\frac{25 c_{2 \theta}}{96}\right) s_{\theta}^{2}+\left(-\frac{25}{96}-\frac{35 c_{2 \theta}}{96}\right) c_{2 \iota} s_{\theta}^{2}\right) s_{\iota}^{2}+\nu\left(\left(-\frac{45}{32}+\frac{25 c_{2 \theta}}{32}\right) s_{\theta}^{2}\right.\right. \\
& \left.\left.+\left(\frac{25}{32}+\frac{35 c_{2 \theta}}{32}\right) c_{2 \iota} s_{\theta}^{2}\right) s_{\iota}^{2}\right]+\cos (4 \Psi)\left(\left(-\frac{25}{12}-\frac{35 c_{2 \theta}}{12}\right) s_{\theta}^{2} s_{\iota}^{4}+\nu\left(\frac{25}{4}+\frac{35 c_{2 \theta}}{4}\right) s_{\theta}^{2} s_{\iota}^{4}\right) \\
& +\cos (\alpha+2 \Psi)\left[c_{\frac{\iota}{2}}^{3}\left(6 s_{2 \theta}-\frac{31}{12} c_{\iota} s_{2 \theta}+\frac{1}{12} c_{2 \iota} s_{2 \theta}-\frac{19 s_{4 \theta}}{48}\right) s_{\frac{\iota}{2}}+\frac{7}{24} c_{\frac{\iota}{2}}^{3} s_{4 \theta} s_{\frac{3 \iota}{2}}-\frac{7}{48} c_{\frac{\iota}{2}}^{3} s_{4 \theta} s_{\frac{5 \iota}{2}}\right. \\
& \left.+\nu\left(c_{\frac{\iota}{2}}^{3}\left(-\frac{16 s_{2 \theta}}{3}+\frac{31}{4} c_{\iota} s_{2 \theta}-\frac{1}{4} c_{2 \iota} s_{2 \theta}+\frac{19 s_{4 \theta}}{16}\right) s_{\frac{\iota}{2}}-\frac{7}{8} c_{\frac{\iota}{2}}^{3} s_{4 \theta} s_{\frac{3 \iota}{2}}+\frac{7}{16} c_{\frac{\iota}{2}}^{3} s_{4 \theta} s_{\frac{5 \iota}{2}}\right)\right], \\
& \hat{h}_{+}^{(3 / 2)}=\left(-3 \pi-\pi c_{2 \theta}\right) c_{\frac{\iota}{2}}^{4} \cos (2 \alpha+2 \Psi)-4 \pi c_{\frac{\iota}{2}}^{3} \cos (\alpha+2 \Psi) s_{2 \theta} s_{\frac{\iota}{2}}+4 \pi c_{\frac{\iota}{2}} \cos (\alpha-2 \Psi) s_{2 \theta} s_{\frac{\iota}{2}}^{3} \\
& +\left(-3 \pi-\pi c_{2 \theta}\right) \cos (2 \alpha-2 \Psi) s_{\frac{\iota}{2}}^{4}-3 \pi \cos (2 \Psi) s_{\theta}^{2} s_{\iota}^{2}+\delta\left[\operatorname { c o s } ( 5 \alpha + 5 \Psi ) \left(\left(-\frac{625}{256}-\frac{625 c_{2 \theta}}{768}\right) c_{\frac{\iota}{2}}^{10} s_{\theta}^{3}\right.\right. \\
& \left.+\nu\left(\frac{625}{128}+\frac{625 c_{2 \theta}}{384}\right) c_{\frac{\iota}{2}}^{10} s_{\theta}^{3}\right)+\cos (\alpha+\Psi)\left[\nu c _ { \frac { \iota } { 2 } } ^ { 2 } \left(-\frac{7449 s_{\theta}}{16384}-\frac{331 s_{3 \theta}}{32768}+c_{2 \iota}\left(\frac{337 s_{\theta}}{12288}-\frac{47 s_{3 \theta}}{8192}-\frac{21 s_{5 \theta}}{8192}\right)\right.\right. \\
& +c_{4 \iota}\left(\frac{7 s_{\theta}}{49152}+\frac{7 s_{3 \theta}}{32768}-\frac{35 s_{5 \theta}}{32768}\right)+c_{3 \iota}\left(-\frac{59 s_{\theta}}{6144}-\frac{91 s_{3 \theta}}{4096}+\frac{7 s_{5 \theta}}{4096}\right)+c_{\iota}\left(\frac{1873 s_{\theta}}{2048}+\frac{19 s_{3 \theta}}{4096}+\frac{35 s_{5 \theta}}{12288}\right) \\
& \left.-\frac{155 s_{5 \theta}}{98304}\right)+c_{\frac{\iota}{2}}^{2}\left(\frac{43723 s_{\theta}}{98304}-\frac{9653 s_{3 \theta}}{65536}+c_{\iota}\left(-\frac{10675 s_{\theta}}{12288}+\frac{1901 s_{3 \theta}}{8192}-\frac{35 s_{5 \theta}}{24576}\right)+c_{3 \iota}\left(\frac{59 s_{\theta}}{12288}+\frac{91 s_{3 \theta}}{8192}-\frac{7 s_{5 \theta}}{8192}\right)\right. \\
& \left.\left.+c_{4 \iota}\left(-\frac{7 s_{\theta}}{98304}-\frac{7 s_{3 \theta}}{65536}+\frac{35 s_{5 \theta}}{65536}\right)+c_{2 \iota}\left(\frac{1103 s_{\theta}}{24576}-\frac{2833 s_{3 \theta}}{16384}+\frac{21 s_{5 \theta}}{16384}\right)+\frac{155 s_{5 \theta}}{196608}\right)\right] \\
& +\cos (3 \alpha+3 \Psi)\left[c _ { \frac { \iota } { 2 } } ^ { 6 } \left(\frac{39249 s_{\theta}}{8192}+\frac{38331 s_{3 \theta}}{16384}+c_{2 \iota}\left(-\frac{1701 s_{\theta}}{8192}-\frac{3159 s_{3 \theta}}{16384}-\frac{3645 s_{5 \theta}}{16384}\right)\right.\right. \\
& \left.+c_{\iota}\left(\frac{2403 s_{\theta}}{2048}-\frac{6399 s_{3 \theta}}{4096}+\frac{2187 s_{5 \theta}}{4096}\right)-\frac{5751 s_{5 \theta}}{16384}\right)+\nu c_{\frac{\iota}{2}}^{6}\left(-\frac{4689 s_{\theta}}{4096}-\frac{24507 s_{3 \theta}}{8192}\right. \\
& \left.\left.+c_{\iota}\left(-\frac{2403 s_{\theta}}{1024}+\frac{6399 s_{3 \theta}}{2048}-\frac{2187 s_{5 \theta}}{2048}\right)+c_{2 \iota}\left(\frac{1701 s_{\theta}}{4096}+\frac{3159 s_{3 \theta}}{8192}+\frac{3645 s_{5 \theta}}{8192}\right)+\frac{5751 s_{5 \theta}}{8192}\right)\right] \\
& +\cos (4 \alpha+5 \Psi)\left(\nu\left(-\frac{11875 c_{\theta}}{384}-\frac{3125 c_{3 \theta}}{384}\right) c_{\frac{\iota}{2}}^{9} s_{\theta}^{2} s_{\frac{\iota}{2}}+\left(\frac{11875 c_{\theta}}{768}+\frac{3125 c_{3 \theta}}{768}\right) c_{\frac{\iota}{2}}^{9} s_{\theta}^{2} s_{\frac{\iota}{2}}\right) \\
& +\cos (4 \alpha+3 \Psi)\left[c_{\frac{\iota}{2}}^{7}\left(\left(-\frac{351 c_{\theta}}{256}+\frac{243}{256} c_{\theta} c_{2 \theta}\right) s_{\theta}^{2}+\left(-\frac{567 c_{\theta}}{256}-\frac{405}{256} c_{\theta} c_{2 \theta}\right) c_{\iota} s_{\theta}^{2}\right) s_{\frac{\iota}{2}}\right.
\end{aligned}
$$




$$
\begin{aligned}
& \left.+\nu c_{\frac{\iota}{2}}^{7}\left(\left(\frac{351 c_{\theta}}{128}-\frac{243}{128} c_{\theta} c_{2 \theta}\right) s_{\theta}^{2}+\left(\frac{567 c_{\theta}}{128}+\frac{405}{128} c_{\theta} c_{2 \theta}\right) c_{\iota} s_{\theta}^{2}\right) s_{\frac{\iota}{2}}\right]+\cos (5 \alpha+3 \Psi)\left(\left(-\frac{243}{256}-\frac{81 c_{2 \theta}}{256}\right) c_{\frac{\iota}{2}}^{8} s_{\theta}^{3} s_{\frac{\iota}{2}}^{2}\right. \\
& \left.+\nu\left(\frac{243}{128}+\frac{81 c_{2 \theta}}{128}\right) c_{\frac{\iota}{2}}^{8} s_{\theta}^{3} s_{\frac{\iota}{2}}^{2}\right)+\cos (\alpha-\Psi)\left[\left(-\frac{43723 s_{\theta}}{98304}+\frac{9653 s_{3 \theta}}{65536}+c_{\iota}\left(-\frac{10675 s_{\theta}}{12288}+\frac{1901 s_{3 \theta}}{8192}-\frac{35 s_{5 \theta}}{24576}\right)\right.\right. \\
& +c_{2 \iota}\left(-\frac{1103 s_{\theta}}{24576}+\frac{2833 s_{3 \theta}}{16384}-\frac{21 s_{5 \theta}}{16384}\right)+c_{3 \iota}\left(\frac{59 s_{\theta}}{12288}+\frac{91 s_{3 \theta}}{8192}-\frac{7 s_{5 \theta}}{8192}\right)+c_{4 \iota}\left(\frac{7 s_{\theta}}{98304}+\frac{7 s_{3 \theta}}{65536}-\frac{35 s_{5 \theta}}{65536}\right) \\
& \left.-\frac{155 s_{5 \theta}}{196608}\right) s_{\frac{\iota}{2}}^{2}+\nu\left(\frac{7449 s_{\theta}}{16384}+\frac{331 s_{3 \theta}}{32768}+c_{4 \iota}\left(-\frac{7 s_{\theta}}{49152}-\frac{7 s_{3 \theta}}{32768}+\frac{35 s_{5 \theta}}{32768}\right)+c_{3 \iota}\left(-\frac{59 s_{\theta}}{6144}-\frac{91 s_{3 \theta}}{4096}+\frac{7 s_{5 \theta}}{4096}\right)\right. \\
& \left.\left.+c_{2 \iota}\left(-\frac{337 s_{\theta}}{12288}+\frac{47 s_{3 \theta}}{8192}+\frac{21 s_{5 \theta}}{8192}\right)+c_{\iota}\left(\frac{1873 s_{\theta}}{2048}+\frac{19 s_{3 \theta}}{4096}+\frac{35 s_{5 \theta}}{12288}\right)+\frac{155 s_{5 \theta}}{98304}\right) s_{\frac{\iota}{2}}^{2}\right] \\
& +\cos (3 \alpha+\Psi)\left[c _ { \frac { \iota } { 2 } } ^ { 4 } \left(\frac{1675 s_{\theta}}{4096}+\frac{825 s_{3 \theta}}{8192}+c_{2 \iota}\left(-\frac{7 s_{\theta}}{4096}-\frac{13 s_{3 \theta}}{8192}-\frac{15 s_{5 \theta}}{8192}\right)+c_{\iota}\left(\frac{27 s_{\theta}}{1024}-\frac{151 s_{3 \theta}}{2048}+\frac{3 s_{5 \theta}}{2048}\right)\right.\right. \\
& \left.-\frac{13 s_{5 \theta}}{8192}\right) s_{\frac{\iota}{2}}^{2}+\nu c_{\frac{\iota}{2}}^{4}\left(\frac{245 s_{\theta}}{2048}-\frac{57 s_{3 \theta}}{4096}+c_{\iota}\left(-\frac{27 s_{\theta}}{512}+\frac{151 s_{3 \theta}}{1024}-\frac{3 s_{5 \theta}}{1024}\right)+c_{2 \iota}\left(\frac{7 s_{\theta}}{2048}+\frac{13 s_{3 \theta}}{4096}+\frac{15 s_{5 \theta}}{4096}\right)\right. \\
& \left.\left.+\frac{13 s_{5 \theta}}{4096}\right) s_{\frac{\iota}{2}}^{2}\right]+\cos (3 \alpha+5 \Psi)\left(c_{\frac{\iota}{2}}^{8}\left(-\frac{4375 s_{\theta}}{1024}-\frac{8125 s_{3 \theta}}{2048}-\frac{9375 s_{5 \theta}}{2048}\right) s_{\frac{\iota}{2}}^{2}\right. \\
& \left.+\nu c_{\frac{L}{2}}^{8}\left(\frac{4375 s_{\theta}}{512}+\frac{8125 s_{3 \theta}}{1024}+\frac{9375 s_{5 \theta}}{1024}\right) s_{\frac{L}{2}}^{2}\right)+\cos (\alpha+3 \Psi)\left[c _ { \frac { L } { 2 } } ^ { 4 } \left(\frac{20475 s_{\theta}}{4096}-\frac{149391 s_{3 \theta}}{8192}\right.\right. \\
& \left.+c_{\iota}\left(\frac{2187 s_{\theta}}{1024}+\frac{10017 s_{3 \theta}}{2048}-\frac{1701 s_{5 \theta}}{2048}\right)+\frac{7371 s_{5 \theta}}{8192}+c_{2 \iota}\left(-\frac{567 s_{\theta}}{4096}-\frac{1701 s_{3 \theta}}{8192}+\frac{8505 s_{5 \theta}}{8192}\right)\right) s_{\frac{\iota}{2}}^{2} \\
& +\nu c_{\frac{\iota}{2}}^{4}\left(-\frac{3195 s_{\theta}}{2048}+\frac{45711 s_{3 \theta}}{4096}+c_{2 \iota}\left(\frac{567 s_{\theta}}{2048}+\frac{1701 s_{3 \theta}}{4096}-\frac{8505 s_{5 \theta}}{4096}\right)-\frac{7371 s_{5 \theta}}{4096}\right. \\
& \left.\left.+c_{\iota}\left(-\frac{2187 s_{\theta}}{512}-\frac{10017 s_{3 \theta}}{1024}+\frac{1701 s_{5 \theta}}{1024}\right)\right) s_{\frac{\iota}{2}}^{2}\right]+\cos (2 \alpha+5 \Psi)\left(\nu\left(-\frac{4375 c_{\theta}}{192}-\frac{625 c_{3 \theta}}{128}-\frac{3125 c_{5 \theta}}{128}\right) c_{\frac{\iota}{2}}^{7} s_{\frac{L}{2}}^{3}\right. \\
& \left.+\left(\frac{4375 c_{\theta}}{384}+\frac{625 c_{3 \theta}}{256}+\frac{3125 c_{5 \theta}}{256}\right) c_{\frac{L}{2}}^{7} s_{\frac{L}{2}}^{3}\right)+\cos (4 \alpha+\Psi)\left[c _ { \frac { L } { 2 } } ^ { 5 } \left(\left(-\frac{37 c_{\theta}}{384}+\frac{1}{384} c_{\theta} c_{2 \theta}\right) s_{\theta}^{2}\right.\right. \\
& \left.\left.+\left(-\frac{7 c_{\theta}}{384}-\frac{5}{384} c_{\theta} c_{2 \theta}\right) c_{\iota} s_{\theta}^{2}\right) s_{\frac{\iota}{2}}^{3}+\nu c_{\frac{\iota}{2}}^{5}\left(\left(\frac{37 c_{\theta}}{192}-\frac{1}{192} c_{\theta} c_{2 \theta}\right) s_{\theta}^{2}+\left(\frac{7 c_{\theta}}{192}+\frac{5}{192} c_{\theta} c_{2 \theta}\right) c_{\iota} s_{\theta}^{2}\right) s_{\frac{\iota}{2}}^{3}\right] \\
& +\cos (5 \alpha+\Psi)\left(\left(-\frac{1}{128}-\frac{c_{2 \theta}}{384}\right) c_{\frac{\iota}{2}}^{6} s_{\theta}^{3} s_{\frac{\iota}{2}}^{4}+\nu\left(\frac{1}{64}+\frac{c_{2 \theta}}{192}\right) c_{\frac{\iota}{2}}^{6} s_{\theta}^{3} s_{\frac{\iota}{2}}^{4}\right)+\cos (3 \alpha-\Psi)\left[\nu c _ { \frac { \iota } { 2 } } ^ { 2 } \left(-\frac{245 s_{\theta}}{2048}+\frac{57 s_{3 \theta}}{4096}\right.\right. \\
& \left.+c_{2 \iota}\left(-\frac{7 s_{\theta}}{2048}-\frac{13 s_{3 \theta}}{4096}-\frac{15 s_{5 \theta}}{4096}\right)+c_{\iota}\left(-\frac{27 s_{\theta}}{512}+\frac{151 s_{3 \theta}}{1024}-\frac{3 s_{5 \theta}}{1024}\right)-\frac{13 s_{5 \theta}}{4096}\right) s_{\frac{\iota}{2}}^{4}+c_{\frac{\iota}{2}}^{2}\left(-\frac{1675 s_{\theta}}{4096}-\frac{825 s_{3 \theta}}{8192}\right. \\
& \left.\left.+c_{\iota}\left(\frac{27 s_{\theta}}{1024}-\frac{151 s_{3 \theta}}{2048}+\frac{3 s_{5 \theta}}{2048}\right)+c_{2 \iota}\left(\frac{7 s_{\theta}}{4096}+\frac{13 s_{3 \theta}}{8192}+\frac{15 s_{5 \theta}}{8192}\right)+\frac{13 s_{5 \theta}}{8192}\right) s_{\frac{\iota}{2}}^{4}\right] \\
& +\cos (\alpha+5 \Psi)\left(\nu c_{\frac{L}{2}}^{6}\left(\frac{4375 s_{\theta}}{768}+\frac{4375 s_{3 \theta}}{512}-\frac{21875 s_{5 \theta}}{512}\right) s_{\frac{L}{2}}^{4}+c_{\frac{L}{2}}^{6}\left(-\frac{4375 s_{\theta}}{1536}-\frac{4375 s_{3 \theta}}{1024}+\frac{21875 s_{5 \theta}}{1024}\right) s_{\frac{\iota}{2}}^{4}\right) \\
& +\cos (\alpha-3 \Psi)\left[c _ { \frac { \iota } { 2 } } ^ { 2 } \left(-\frac{20475 s_{\theta}}{4096}+\frac{149391 s_{3 \theta}}{8192}+c_{2 \iota}\left(\frac{567 s_{\theta}}{4096}+\frac{1701 s_{3 \theta}}{8192}-\frac{8505 s_{5 \theta}}{8192}\right)\right.\right. \\
& \left.+c_{\iota}\left(\frac{2187 s_{\theta}}{1024}+\frac{10017 s_{3 \theta}}{2048}-\frac{1701 s_{5 \theta}}{2048}\right)-\frac{7371 s_{5 \theta}}{8192}\right) s_{\frac{\iota}{2}}^{4}+\nu c_{\frac{\iota}{2}}^{2}\left(\frac{3195 s_{\theta}}{2048}-\frac{45711 s_{3 \theta}}{4096}+\frac{7371 s_{5 \theta}}{4096}\right. \\
& \left.\left.+c_{\iota}\left(-\frac{2187 s_{\theta}}{512}-\frac{10017 s_{3 \theta}}{1024}+\frac{1701 s_{5 \theta}}{1024}\right)+c_{2 \iota}\left(-\frac{567 s_{\theta}}{2048}-\frac{1701 s_{3 \theta}}{4096}+\frac{8505 s_{5 \theta}}{4096}\right)\right) s_{\frac{\iota}{2}}^{4}\right] \\
& +\cos (4 \alpha-\Psi)\left[\nu c_{\frac{L}{2}}^{3}\left(\left(\frac{37 c_{\theta}}{192}-\frac{1}{192} c_{\theta} c_{2 \theta}\right) s_{\theta}^{2}+\left(-\frac{7 c_{\theta}}{192}-\frac{5}{192} c_{\theta} c_{2 \theta}\right) c_{\iota} s_{\theta}^{2}\right) s_{\frac{L}{2}}^{5}+c_{\frac{\iota}{2}}^{3}\left(\left(-\frac{37 c_{\theta}}{384}+\frac{1}{384} c_{\theta} c_{2 \theta}\right) s_{\theta}^{2}\right.\right. \\
& \left.\left.+\left(\frac{7 c_{\theta}}{384}+\frac{5}{384} c_{\theta} c_{2 \theta}\right) c_{\iota} s_{\theta}^{2}\right) s_{\frac{\iota}{2}}^{5}\right]+\cos (5 \alpha-\Psi)\left(\nu\left(-\frac{1}{64}-\frac{c_{2 \theta}}{192}\right) c_{\frac{\iota}{2}}^{4} s_{\theta}^{3} s_{\frac{\iota}{2}}^{6}+\left(\frac{1}{128}+\frac{c_{2 \theta}}{384}\right) c_{\frac{\iota}{2}}^{4} s_{\theta}^{3} s_{\frac{\iota}{2}}^{6}\right) \\
& +\cos (3 \alpha-3 \Psi)\left[\nu \left(\left(\frac{14067}{4096}+\frac{4689 c_{2 \theta}}{1024}-\frac{5751 c_{4 \theta}}{4096}\right) s_{\theta}+\left(-\frac{297}{1024}+\frac{1053 c_{2 \theta}}{256}-\frac{2187 c_{4 \theta}}{1024}\right) c_{\iota} s_{\theta}\right.\right.
\end{aligned}
$$




$$
\begin{aligned}
& \left.+\left(-\frac{5103}{4096}-\frac{1701 c_{2 \theta}}{1024}-\frac{3645 c_{4 \theta}}{4096}\right) c_{2 \iota} s_{\theta}\right) s_{\frac{\iota}{2}}^{6}+\left(\left(-\frac{55539}{8192}-\frac{8145 c_{2 \theta}}{2048}+\frac{5751 c_{4 \theta}}{8192}\right) s_{\theta}\right. \\
& \left.\left.+\left(\frac{297}{2048}-\frac{1053 c_{2 \theta}}{512}+\frac{2187 c_{4 \theta}}{2048}\right) c_{\iota} s_{\theta}+\left(\frac{5103}{8192}+\frac{1701 c_{2 \theta}}{2048}+\frac{3645 c_{4 \theta}}{8192}\right) c_{2 \iota} s_{\theta}\right) s_{\frac{\iota}{2}}^{6}\right] \\
& +\cos (\alpha-5 \Psi)\left(c_{\frac{L}{2}}^{4}\left(\frac{4375 s_{\theta}}{1536}+\frac{4375 s_{3 \theta}}{1024}-\frac{21875 s_{5 \theta}}{1024}\right) s_{\frac{L}{2}}^{6}+\nu c_{\frac{L}{2}}^{4}\left(-\frac{4375 s_{\theta}}{768}-\frac{4375 s_{3 \theta}}{512}+\frac{21875 s_{5 \theta}}{512}\right) s_{\frac{L}{2}}^{6}\right) \\
& +\cos (2 \alpha-5 \Psi)\left(\nu\left(-\frac{4375 c_{\theta}}{192}-\frac{625 c_{3 \theta}}{128}-\frac{3125 c_{5 \theta}}{128}\right) c_{\frac{L}{2}}^{3} s_{\frac{L}{2}}^{7}+\left(\frac{4375 c_{\theta}}{384}+\frac{625 c_{3 \theta}}{256}+\frac{3125 c_{5 \theta}}{256}\right) c_{\frac{L}{2}}^{3} s_{\frac{L}{2}}^{7}\right) \\
& +\cos (4 \alpha-3 \Psi)\left[\nu c_{\frac{\iota}{2}}\left(\left(\frac{351 c_{\theta}}{128}-\frac{243}{128} c_{\theta} c_{2 \theta}\right) s_{\theta}^{2}+\left(-\frac{567 c_{\theta}}{128}-\frac{405}{128} c_{\theta} c_{2 \theta}\right) c_{\iota} s_{\theta}^{2}\right) s_{\frac{L}{2}}^{7}\right. \\
& \left.+c_{\frac{\iota}{2}}\left(\left(-\frac{351 c_{\theta}}{256}+\frac{243}{256} c_{\theta} c_{2 \theta}\right) s_{\theta}^{2}+\left(\frac{567 c_{\theta}}{256}+\frac{405}{256} c_{\theta} c_{2 \theta}\right) c_{\iota} s_{\theta}^{2}\right) s_{\frac{\iota}{2}}^{7}\right] \\
& +\cos (5 \alpha-3 \Psi)\left(\nu\left(-\frac{243}{128}-\frac{81 c_{2 \theta}}{128}\right) c_{\frac{L}{2}}^{2} s_{\theta}^{3} s_{\frac{L}{2}}^{8}+\left(\frac{243}{256}+\frac{81 c_{2 \theta}}{256}\right) c_{\frac{L}{2}}^{2} s_{\theta}^{3} s_{\frac{L}{2}}^{8}\right) \\
& +\cos (3 \alpha-5 \Psi)\left(\nu c_{\frac{L}{2}}^{2}\left(-\frac{4375 s_{\theta}}{512}-\frac{8125 s_{3 \theta}}{1024}-\frac{9375 s_{5 \theta}}{1024}\right) s_{\frac{L}{2}}^{8}+c_{\frac{L}{2}}^{2}\left(\frac{4375 s_{\theta}}{1024}+\frac{8125 s_{3 \theta}}{2048}+\frac{9375 s_{5 \theta}}{2048}\right) s_{\frac{L}{2}}^{8}\right) \\
& +\cos (4 \alpha-5 \Psi)\left(\nu\left(-\frac{11875 c_{\theta}}{384}-\frac{3125 c_{3 \theta}}{384}\right) c_{\frac{\iota}{2}} s_{\theta}^{2} s_{\frac{\iota}{2}}^{9}+\left(\frac{11875 c_{\theta}}{768}+\frac{3125 c_{3 \theta}}{768}\right) c_{\frac{\iota}{2}} s_{\theta}^{2} s_{\frac{\iota}{2}}^{9}\right) \\
& +\cos (5 \alpha-5 \Psi)\left(\nu\left(-\frac{625}{128}-\frac{625 c_{2 \theta}}{384}\right) s_{\theta}^{3} s_{\frac{\frac{L}{2}}{10}}^{10}+\left(\frac{625}{256}+\frac{625 c_{2 \theta}}{768}\right) s_{\theta}^{3} s_{\frac{\frac{L}{2}}{10}}^{10}\right) \\
& +\cos (3 \Psi)\left[\nu\left(\left(\frac{10197 c_{\theta}}{2048}-\frac{3969 c_{\theta} c_{2 \theta}}{2048}\right) s_{\theta}^{2}+\left(-\frac{1701 c_{\theta}}{2048}-\frac{5103 c_{\theta} c_{2 \theta}}{2048}\right) c_{2 \iota} s_{\theta}^{2}\right) s_{\iota}^{3}\right. \\
& \left.+\left(\left(-\frac{44757 c_{\theta}}{4096}+\frac{3969 c_{\theta} c_{2 \theta}}{4096}\right) s_{\theta}^{2}+\left(\frac{1701 c_{\theta}}{4096}+\frac{5103 c_{\theta} c_{2 \theta}}{4096}\right) c_{2 \iota} s_{\theta}^{2}\right) s_{\iota}^{3}\right] \\
& +\cos (5 \Psi)\left(\nu\left(-\frac{21875 c_{\theta}}{2048}-\frac{13125 c_{3 \theta}}{2048}\right) s_{\theta}^{2} s_{\iota}^{5}+\left(\frac{21875 c_{\theta}}{4096}+\frac{13125 c_{3 \theta}}{4096}\right) s_{\theta}^{2} s_{\iota}^{5}\right) \\
& +\cos (2 \alpha+3 \Psi)\left[\left(-\frac{37071 c_{\theta} c_{2 \theta}}{16384}+c_{\theta}\left(-\frac{7641}{8192}+\frac{567 c_{4 \theta}}{32768}\right)+\left(-\frac{10917 c_{\theta}}{8192}-\frac{2835 c_{\theta} c_{2 \theta}}{1024}\right) c_{\iota}\right.\right. \\
& \left.+\left(-\frac{10089 c_{\theta}}{16384}+\frac{135 c_{\theta} c_{2 \theta}}{8192}\right) c_{2 \iota}+\frac{513 c_{\theta} c_{3 \iota}}{8192}+\frac{567 c_{\theta} c_{4 \iota}}{32768}\right) s_{\iota}-\frac{81 c_{\theta} c_{4 \theta} s_{2 \iota}}{8192}+\frac{1053 c_{\theta} c_{4 \theta} s_{3 \iota}}{65536} \\
& +\left(\frac{2565 c_{3 \theta}}{32768}+\frac{729 c_{5 \theta}}{32768}\right) s_{4 \iota}+\left(\frac{243 c_{3 \theta}}{131072}+\frac{1215 c_{5 \theta}}{131072}\right) s_{5 \iota}+\nu\left[\left(\frac{5967 c_{\theta} c_{2 \theta}}{8192}+c_{\theta}\left(\frac{2457}{4096}-\frac{567 c_{4 \theta}}{16384}\right)\right.\right. \\
& \left.+\left(\frac{4005 c_{\theta}}{4096}+\frac{243}{512} c_{\theta} c_{2 \theta}\right) c_{\iota}+\left(\frac{6633 c_{\theta}}{8192}-\frac{5319 c_{\theta} c_{2 \theta}}{4096}\right) c_{2 \iota}-\frac{513 c_{\theta} c_{3 \iota}}{4096}-\frac{567 c_{\theta} c_{4 \iota}}{16384}\right) s_{\iota}+\frac{81 c_{\theta} c_{4 \theta} s_{2 \iota}}{4096} \\
& \left.\left.-\frac{1053 c_{\theta} c_{4 \theta} s_{3 \iota}}{32768}+\left(-\frac{2565 c_{3 \theta}}{16384}-\frac{729 c_{5 \theta}}{16384}\right) s_{4 \iota}+\left(-\frac{243 c_{3 \theta}}{65536}-\frac{1215 c_{5 \theta}}{65536}\right) s_{5 \iota}\right]\right] \\
& +\cos (2 \alpha-3 \Psi)\left[\left(-\frac{18603 c_{\theta} c_{2 \theta}}{8192}+c_{\theta}\left(-\frac{20475}{32768}+\frac{567 c_{4 \theta}}{32768}\right)\right) s_{\iota}+\left(\frac{2835 c_{\theta} c_{2 \theta}}{2048}+c_{\theta}\left(\frac{5715}{8192}+\frac{81 c_{4 \theta}}{8192}\right)\right) s_{2 \iota}\right. \\
& +\left(\frac{135 c_{\theta} c_{2 \theta}}{16384}+c_{\theta}\left(-\frac{20745}{65536}+\frac{1053 c_{4 \theta}}{65536}\right)\right) s_{3 \iota}+\left(-\frac{513 c_{\theta}}{16384}-\frac{2565 c_{3 \theta}}{32768}-\frac{729 c_{5 \theta}}{32768}\right) s_{4 \iota} \\
& +\left(\frac{567 c_{\theta}}{65536}+\frac{243 c_{3 \theta}}{131072}+\frac{1215 c_{5 \theta}}{131072}\right) s_{5 \iota}+\nu\left[\left(\frac{5643 c_{\theta} c_{2 \theta}}{4096}+c_{\theta}\left(\frac{3195}{16384}-\frac{567 c_{4 \theta}}{16384}\right)\right) s_{\iota}+\left(-\frac{243 c_{\theta} c_{2 \theta}}{1024}\right.\right. \\
& \left.+c_{\theta}\left(-\frac{2259}{4096}-\frac{81 c_{4 \theta}}{4096}\right)\right) s_{2 \iota}+\left(-\frac{5319 c_{\theta} c_{2 \theta}}{8192}+c_{\theta}\left(\frac{13833}{32768}-\frac{1053 c_{4 \theta}}{32768}\right)\right) s_{3 \iota} \\
& \left.\left.+\left(\frac{513 c_{\theta}}{8192}+\frac{2565 c_{3 \theta}}{16384}+\frac{729 c_{5 \theta}}{16384}\right) s_{4 \iota}+\left(-\frac{567 c_{\theta}}{32768}-\frac{243 c_{3 \theta}}{65536}-\frac{1215 c_{5 \theta}}{65536}\right) s_{5 \iota}\right]\right]+\cos (2 \alpha+\Psi)\left[\left(\frac{319 c_{\theta} c_{2 \theta}}{24576}\right.\right. \\
& \left.+c_{\theta}\left(\frac{871}{4096}+\frac{c_{4 \theta}}{49152}\right)+\left(\frac{933 c_{\theta}}{4096}+\frac{133 c_{\theta} c_{2 \theta}}{1536}\right) c_{\iota}+\left(\frac{625 c_{\theta}}{24576}+\frac{211 c_{\theta} c_{2 \theta}}{4096}\right) c_{2 \iota}-\frac{11 c_{\theta} c_{3 \iota}}{12288}-\frac{7 c_{\theta} c_{4 \iota}}{49152}\right) s_{\iota}
\end{aligned}
$$




$$
\begin{aligned}
& -\frac{c_{\theta} c_{4 \theta} s_{2 \iota}}{12288}+\frac{c_{\theta} c_{4 \theta} s_{3 \iota}}{32768}+\left(-\frac{45 c_{3 \theta}}{16384}-\frac{c_{5 \theta}}{16384}\right) s_{4 \iota}+\left(-\frac{c_{3 \theta}}{65536}-\frac{5 c_{5 \theta}}{65536}\right) s_{5 \iota}+\nu\left[\left(\frac{257 c_{\theta} c_{2 \theta}}{12288}\right.\right. \\
& \left.+c_{\theta}\left(-\frac{1493}{6144}-\frac{c_{4 \theta}}{24576}\right)+\left(-\frac{1391 c_{\theta}}{6144}+\frac{11}{768} c_{\theta} c_{2 \theta}\right) c_{\iota}+\left(-\frac{49 c_{\theta}}{12288}+\frac{77 c_{\theta} c_{2 \theta}}{2048}\right) c_{2 \iota}+\frac{11 c_{\theta} c_{3 \iota}}{6144}+\frac{7 c_{\theta} c_{4 \iota}}{24576}\right) s_{\iota} \\
& \left.\left.+\frac{c_{\theta} c_{4 \theta} s_{2 \iota}}{6144}-\frac{c_{\theta} c_{4 \theta} s_{3 \iota}}{16384}+\left(\frac{45 c_{3 \theta}}{8192}+\frac{c_{5 \theta}}{8192}\right) s_{4 \iota}+\left(\frac{c_{3 \theta}}{32768}+\frac{5 c_{5 \theta}}{32768}\right) s_{5 \iota}\right]\right]+\cos (2 \alpha-\Psi)\left[\left(-\frac{157 c_{\theta} c_{2 \theta}}{12288}\right.\right. \\
& \left.+c_{\theta}\left(\frac{9827}{49152}+\frac{c_{4 \theta}}{49152}\right)\right) s_{\iota}+\left(-\frac{133 c_{\theta} c_{2 \theta}}{3072}+c_{\theta}\left(-\frac{1405}{12288}+\frac{c_{4 \theta}}{12288}\right)\right) s_{2 \iota}+\left(\frac{211 c_{\theta} c_{2 \theta}}{8192}\right. \\
& \left.+c_{\theta}\left(\frac{419}{32768}+\frac{c_{4 \theta}}{32768}\right)\right) s_{3 \iota}+\left(\frac{11 c_{\theta}}{24576}+\frac{45 c_{3 \theta}}{16384}+\frac{c_{5 \theta}}{16384}\right) s_{4 \iota}+\left(-\frac{7 c_{\theta}}{98304}-\frac{c_{3 \theta}}{65536}-\frac{5 c_{5 \theta}}{65536}\right) s_{5 \iota} \\
& +\nu\left[\left(\frac{13 c_{\theta} c_{2 \theta}}{6144}+c_{\theta}\left(-\frac{5923}{24576}-\frac{c_{4 \theta}}{24576}\right)\right) s_{\iota}+\left(-\frac{11 c_{\theta} c_{2 \theta}}{1536}+c_{\theta}\left(\frac{701}{6144}-\frac{c_{4 \theta}}{6144}\right)\right) s_{2 \iota}\right. \\
& +\left(\frac{77 c_{\theta} c_{2 \theta}}{4096}+c_{\theta}\left(-\frac{35}{16384}-\frac{c_{4 \theta}}{16384}\right)\right) s_{3 \iota}+\left(-\frac{11 c_{\theta}}{12288}-\frac{45 c_{3 \theta}}{8192}-\frac{c_{5 \theta}}{8192}\right) s_{4 \iota} \\
& \left.\left.+\left(\frac{7 c_{\theta}}{49152}+\frac{c_{3 \theta}}{32768}+\frac{5 c_{5 \theta}}{32768}\right) s_{5 \iota}\right]\right]+\cos (\Psi)\left[\left(-\frac{341 c_{\theta}}{8192}+\frac{c_{\theta} c_{2 \theta}}{8192}\right) s_{\theta}^{2} s_{\iota}+\left(-\frac{3411 c_{\theta}}{16384}+\frac{7 c_{\theta} c_{2 \theta}}{16384}\right) s_{\theta}^{2} s_{3 \iota}\right. \\
& +\left(\frac{35 c_{\theta}}{32768}+\frac{21 c_{3 \theta}}{32768}\right) s_{\theta}^{2} s_{5 \iota}+\nu\left(\left(-\frac{43 c_{\theta}}{4096}-\frac{c_{\theta} c_{2 \theta}}{4096}\right) s_{\theta}^{2} s_{\iota}+\left(-\frac{429 c_{\theta}}{8192}-\frac{7 c_{\theta} c_{2 \theta}}{8192}\right) s_{\theta}^{2} s_{3 \iota}\right. \\
& \left.\left.\left.+\left(-\frac{35 c_{\theta}}{16384}-\frac{21 c_{3 \theta}}{16384}\right) s_{\theta}^{2} s_{5 \iota}\right)\right]\right] \\
& \hat{h}_{+}^{(1, \mathrm{SO})}=\cos (\alpha+\Psi)\left(\chi_{a}^{x} c_{\theta} c_{\frac{\iota}{2}}^{2}-\chi_{a}^{z} c_{\frac{\iota}{2}}^{2} s_{\theta}\right)+\cos (\alpha-\Psi)\left(\chi_{a}^{x}\left(\frac{c_{\theta}}{2}-\frac{c_{\theta} c_{\iota}}{2}\right)-\chi_{a}^{z} s_{\theta} s_{\frac{\iota}{2}}^{2}\right)-\chi_{a}^{y} c_{\theta} s_{\frac{\iota}{2}}^{2} \sin (\alpha-\Psi) \\
& -\chi_{a}^{y} s_{\theta} s_{\iota} \sin (\Psi)-\chi_{a}^{y} c_{\theta} c_{\frac{\iota}{2}}^{2} \sin (\alpha+\Psi)+\delta\left[\cos (\alpha+\Psi)\left(\chi_{s}^{x} c_{\theta} c_{\frac{\iota}{2}}^{2}-\chi_{s}^{z} c_{\frac{L}{2}}^{2} s_{\theta}\right)\right. \\
& \left.+\cos (\alpha-\Psi)\left(\chi_{s}^{x}\left(\frac{c_{\theta}}{2}-\frac{c_{\theta} c_{\iota}}{2}\right)-\chi_{s}^{z} s_{\theta} s_{\frac{\iota}{2}}^{2}\right)-\chi_{s}^{y} c_{\theta} s_{\frac{\iota}{2}}^{2} \sin (\alpha-\Psi)-\chi_{s}^{y} s_{\theta} s_{\iota} \sin (\Psi)-\chi_{s}^{y} c_{\theta} c_{\frac{\iota}{2}}^{2} \sin (\alpha+\Psi)\right] \\
& \hat{h}_{+}^{(3 / 2, \mathrm{SO})}=\chi_{s}^{x}\left(2 c_{\theta} c_{\iota}^{3} s_{\theta}-\nu c_{\theta} c_{\iota}^{3} s_{\theta}\right)+\cos (2 \alpha+2 \Psi)\left[\chi _ { s } ^ { z } \left[\nu c_{\frac{\iota}{2}}^{4}\left(-\frac{5}{2}-\frac{7 c_{2 \theta}}{2}+\left(\frac{1}{2}+\frac{c_{2 \theta}}{6}\right) c_{\iota}\right)\right.\right. \\
& \left.\left.+c_{\frac{\iota}{2}}^{4}\left(-3-c_{2 \theta}+\left(5+\frac{5 c_{2 \theta}}{3}\right) c_{\iota}\right)\right]+\chi_{s}^{x}\left(c_{\frac{\iota}{2}}^{4}\left(\frac{7 s_{2 \theta}}{3}-\frac{10}{3} c_{\iota} s_{2 \theta}\right)+\nu c_{\frac{\iota}{2}}^{4}\left(-\frac{19 s_{2 \theta}}{6}-\frac{1}{3} c_{\iota} s_{2 \theta}\right)\right)\right] \\
& +\chi_{s}^{x} \cos (3 \alpha+2 \Psi)\left(\nu\left(\frac{1}{2}+\frac{c_{2 \theta}}{6}\right) c_{\frac{\iota}{2}}^{5} s_{\frac{\iota}{2}}+\left(5+\frac{5 c_{2 \theta}}{3}\right) c_{\frac{\iota}{2}}^{5} s_{\frac{\iota}{2}}\right)+\chi_{s}^{x} \cos (3 \alpha-2 \Psi)\left(\nu\left(\frac{1}{2}+\frac{c_{2 \theta}}{6}\right) c_{\frac{\iota}{2}} s_{\frac{\iota}{2}}^{5}\right. \\
& \left.+\left(5+\frac{5 c_{2 \theta}}{3}\right) c_{\frac{\iota}{2}} s_{\frac{\iota}{2}}^{5}\right)+\cos (\alpha+2 \Psi)\left[\chi _ { s } ^ { x } \left[\nu c_{\frac{\iota}{2}}^{3}\left(-\frac{17}{4}+\frac{79 c_{2 \theta}}{12}+\left(-\frac{1}{4}+\frac{7 c_{2 \theta}}{12}\right) c_{\iota}\right) s_{\frac{\iota}{2}}\right.\right. \\
& \left.\left.+c_{\frac{\iota}{2}}^{3}\left(\frac{3}{2}-\frac{13 c_{2 \theta}}{6}+\left(-\frac{5}{2}+\frac{35 c_{2 \theta}}{6}\right) c_{\iota}\right) s_{\frac{\iota}{2}}\right]+\chi_{s}^{z}\left(\nu c_{\frac{\iota}{2}}^{3}\left(-7 s_{2 \theta}+\frac{2}{3} c_{\iota} s_{2 \theta}\right) s_{\frac{\iota}{2}}+c_{\frac{\iota}{2}}^{3}\left(-2 s_{2 \theta}+\frac{20}{3} c_{\iota} s_{2 \theta}\right) s_{\frac{\iota}{2}}\right)\right] \\
& +\cos (\alpha-2 \Psi)\left[\chi_{s}^{x}\left[c_{\frac{\iota}{2}}\left(\frac{3}{2}-\frac{13 c_{2 \theta}}{6}+\left(\frac{5}{2}-\frac{35 c_{2 \theta}}{6}\right) c_{\iota}\right) s_{\frac{\iota}{2}}^{3}+\nu c_{\frac{\iota}{2}}\left(-\frac{17}{4}+\frac{79 c_{2 \theta}}{12}+\left(\frac{1}{4}-\frac{7 c_{2 \theta}}{12}\right) c_{\iota}\right) s_{\frac{\iota}{2}}^{3}\right]\right. \\
& \left.+\chi_{s}^{z}\left(c_{\frac{\iota}{2}}\left(-2 s_{2 \theta}-\frac{20}{3} c_{\iota} s_{2 \theta}\right) s_{\frac{\iota}{2}}^{3}+\nu c_{\frac{\iota}{2}}\left(-7 s_{2 \theta}-\frac{2}{3} c_{\iota} s_{2 \theta}\right) s_{\frac{\iota}{2}}^{3}\right)\right]+\cos (2 \alpha-2 \Psi)\left[\chi _ { s } ^ { z } \left[\nu \left(\frac{5}{2}+\frac{7 c_{2 \theta}}{2}\right.\right.\right. \\
& \left.\left.+\left(\frac{1}{2}+\frac{c_{2 \theta}}{6}\right) c_{\iota}\right) s_{\frac{\iota}{2}}^{4}+\left(3+c_{2 \theta}+\left(5+\frac{5 c_{2 \theta}}{3}\right) c_{\iota}\right) s_{\frac{\iota}{2}}^{4}\right]+\chi_{s}^{x}\left(\left(-\frac{7 s_{2 \theta}}{3}-\frac{10}{3} c_{\iota} s_{2 \theta}\right) s_{\frac{\iota}{2}}^{4}\right. \\
& \left.\left.+\nu\left(\frac{19 s_{2 \theta}}{6}-\frac{1}{3} c_{\iota} s_{2 \theta}\right) s_{\frac{\iota}{2}}^{4}\right)\right]+\chi_{s}^{z}\left(-3 c_{\iota} s_{\theta}^{2} s_{\iota}^{2}+\frac{3}{2} \nu c_{\iota} s_{\theta}^{2} s_{\iota}^{2}\right)+\chi_{s}^{x} \cos (3 \alpha)\left(\nu\left(-\frac{3}{8}-\frac{c_{2 \theta}}{8}\right) s_{\iota}^{3}+\left(\frac{3}{4}+\frac{c_{2 \theta}}{4}\right) s_{\iota}^{3}\right) \\
& +\cos (2 \Psi)\left(\chi_{s}^{x}\left(\frac{10}{3} c_{\theta} c_{\iota} s_{\theta} s_{\iota}^{2}+\frac{1}{3} \nu c_{\theta} c_{\iota} s_{\theta} s_{\iota}^{2}\right)+\chi_{s}^{z}\left(5 c_{\iota} s_{\theta}^{2} s_{\iota}^{2}+\frac{1}{2} \nu c_{\iota} s_{\theta}^{2} s_{\iota}^{2}\right)\right)+\cos (2 \alpha)\left[\chi _ { s } ^ { z } \left(\nu\left(-\frac{3}{4}-\frac{c_{2 \theta}}{4}\right) c_{\iota} s_{\iota}^{2}\right.\right.
\end{aligned}
$$




$$
\begin{aligned}
& \left.\left.+\left(\frac{3}{2}+\frac{c_{2 \theta}}{2}\right) c_{\iota} s_{\iota}^{2}\right)+\chi_{s}^{x}\left(-c_{\iota} s_{2 \theta} s_{\iota}^{2}+\frac{1}{2} \nu c_{\iota} s_{2 \theta} s_{\iota}^{2}\right)\right]+\cos (\alpha)\left[\chi _ { s } ^ { x } \left(-\frac{11}{16} c_{2 \theta} s_{\iota}-\frac{3 s_{\iota}^{3}}{4}-\frac{7}{16} c_{2 \theta} s_{3 \iota}\right.\right. \\
& \left.\left.+\nu\left(\frac{11}{32} c_{2 \theta} s_{\iota}+\frac{3 s_{\iota}^{3}}{8}+\frac{7}{32} c_{2 \theta} s_{3 \iota}\right)\right)+\chi_{s}^{z}\left(\frac{1}{2} s_{2 \theta} s_{\iota}-\frac{1}{2} s_{2 \theta} s_{3 \iota}+\nu\left(-\frac{1}{4} s_{2 \theta} s_{\iota}+\frac{1}{4} s_{2 \theta} s_{3 \iota}\right)\right)\right]+\chi_{s}^{y}\left[\left(\frac{15}{8}-\frac{3 c_{2 \theta}}{8}\right.\right. \\
& \left.\left.+\left(\frac{9}{8}-\frac{5 c_{2 \theta}}{8}\right) c_{2 \iota}\right) s_{\iota}+\nu\left(-\frac{15}{16}+\frac{3 c_{2 \theta}}{16}+\left(-\frac{9}{16}+\frac{5 c_{2 \theta}}{16}\right) c_{2 \iota}\right) s_{\iota}\right] \sin (\alpha) \\
& +\chi_{s}^{y}\left(-2 c_{\theta} c_{\iota} s_{\theta} s_{\iota}^{2}+\nu c_{\theta} c_{\iota} s_{\theta} s_{\iota}^{2}\right) \sin (2 \alpha)+\chi_{s}^{y}\left(\nu\left(-\frac{3}{8}-\frac{c_{2 \theta}}{8}\right) s_{\iota}^{3}+\left(\frac{3}{4}+\frac{c_{2 \theta}}{4}\right) s_{\iota}^{3}\right) \sin (3 \alpha) \\
& +\chi_{s}^{y}\left[c_{\frac{\iota}{2}}\left(\frac{5}{2}-\frac{11 c_{2 \theta}}{6}+\left(\frac{15}{2}-\frac{25 c_{2 \theta}}{6}\right) c_{\iota}\right) s_{\frac{\iota}{2}}^{3}+\nu c_{\frac{\iota}{2}}\left(\frac{1}{4}-\frac{31 c_{2 \theta}}{12}+\left(\frac{3}{4}-\frac{5 c_{2 \theta}}{12}\right) c_{\iota}\right) s_{\frac{\iota}{2}}^{3}\right] \sin (\alpha-2 \Psi) \\
& +\chi_{s}^{y}\left(\left(-\frac{7 s_{2 \theta}}{3}-\frac{10}{3} c_{\iota} s_{2 \theta}\right) s_{\frac{\iota}{2}}^{4}+\nu\left(-\frac{5 s_{2 \theta}}{6}-\frac{1}{3} c_{\iota} s_{2 \theta}\right) s_{\frac{\iota}{2}}^{4}\right) \sin (2 \alpha-2 \Psi)+\chi_{s}^{y}\left(\nu\left(\frac{1}{2}+\frac{c_{2 \theta}}{6}\right) c_{\frac{\iota}{2}} s_{\frac{L}{2}}^{5}\right. \\
& \left.+\left(5+\frac{5 c_{2 \theta}}{3}\right) c_{\frac{\iota}{2}} s_{\frac{\iota}{2}}^{5}\right) \sin (3 \alpha-2 \Psi)+\chi_{s}^{y}\left(-\frac{1}{3} c_{\theta} s_{\theta} s_{\iota}^{2}-\frac{11}{6} \nu c_{\theta} s_{\theta} s_{\iota}^{2}\right) \sin (2 \Psi)+\chi_{s}^{y}\left[\nu c _ { \frac { \iota } { 2 } } ^ { 3 } \left(\frac{1}{4}-\frac{31 c_{2 \theta}}{12}\right.\right. \\
& \left.\left.+\left(-\frac{3}{4}+\frac{5 c_{2 \theta}}{12}\right) c_{\iota}\right) s_{\frac{\iota}{2}}+c_{\frac{\iota}{2}}^{3}\left(\frac{5}{2}-\frac{11 c_{2 \theta}}{6}+\left(-\frac{15}{2}+\frac{25 c_{2 \theta}}{6}\right) c_{\iota}\right) s_{\frac{\iota}{2}}\right] \sin (\alpha+2 \Psi)+\chi_{s}^{y}\left(c_{\frac{\iota}{2}}^{4}\left(\frac{7 s_{2 \theta}}{3}-\frac{10}{3} c_{\iota} s_{2 \theta}\right)\right. \\
& \left.+\nu c_{\frac{L}{2}}^{4}\left(\frac{5 s_{2 \theta}}{6}-\frac{1}{3} c_{\iota} s_{2 \theta}\right)\right) \sin (2 \alpha+2 \Psi)+\chi_{s}^{y}\left(\nu\left(\frac{1}{2}+\frac{c_{2 \theta}}{6}\right) c_{\frac{\iota}{2}}^{5} s_{\frac{\iota}{2}}+\left(5+\frac{5 c_{2 \theta}}{3}\right) c_{\frac{\iota}{2}}^{5} s_{\frac{\iota}{2}}\right) \sin (3 \alpha+2 \Psi) \\
& +\delta\left[2 \chi_{a}^{x} c_{\theta} c_{\iota}^{3} s_{\theta}+\cos (2 \alpha+2 \Psi)\left[\chi_{a}^{z} c_{\frac{\iota}{2}}^{4}\left(-3-c_{2 \theta}+\left(5+\frac{5 c_{2 \theta}}{3}\right) c_{\iota}\right)+\chi_{a}^{x} c_{\frac{\iota}{2}}^{4}\left(\frac{7 s_{2 \theta}}{3}-\frac{10}{3} c_{\iota} s_{2 \theta}\right)\right]\right. \\
& +\chi_{a}^{x}\left(5+\frac{5 c_{2 \theta}}{3}\right) c_{\frac{\iota}{2}}^{5} \cos (3 \alpha+2 \Psi) s_{\frac{\iota}{2}}+\chi_{a}^{x}\left(5+\frac{5 c_{2 \theta}}{3}\right) c_{\frac{\iota}{2}} \cos (3 \alpha-2 \Psi) s_{\frac{\iota}{2}}^{5}+\cos (\alpha+2 \Psi)\left[\chi _ { a } ^ { x } c _ { \frac { \iota } { 2 } } ^ { 3 } \left(\frac{3}{2}-\frac{13 c_{2 \theta}}{6}\right.\right. \\
& \left.\left.+\left(-\frac{5}{2}+\frac{35 c_{2 \theta}}{6}\right) c_{\iota}\right) s_{\frac{\iota}{2}}+\chi_{a}^{z} c_{\frac{\iota}{2}}^{3}\left(-2 s_{2 \theta}+\frac{20}{3} c_{\iota} s_{2 \theta}\right) s_{\frac{\iota}{2}}\right]+\cos (\alpha-2 \Psi)\left[\chi _ { a } ^ { x } c _ { \frac { \iota } { 2 } } \left(\frac{3}{2}-\frac{13 c_{2 \theta}}{6}\right.\right. \\
& \left.\left.+\left(\frac{5}{2}-\frac{35 c_{2 \theta}}{6}\right) c_{\iota}\right) s_{\frac{\iota}{2}}^{3}+\chi_{a}^{z} c_{\frac{\iota}{2}}\left(-2 s_{2 \theta}-\frac{20}{3} c_{\iota} s_{2 \theta}\right) s_{\frac{\iota}{2}}^{3}\right]+\cos (2 \alpha-2 \Psi)\left[\chi_{a}^{z}\left(3+c_{2 \theta}+\left(5+\frac{5 c_{2 \theta}}{3}\right) c_{\iota}\right) s_{\frac{\iota}{2}}^{4}\right. \\
& \left.+\chi_{a}^{x}\left(-\frac{7 s_{2 \theta}}{3}-\frac{10}{3} c_{\iota} s_{2 \theta}\right) s_{\frac{\iota}{2}}^{4}\right]-3 \chi_{a}^{z} c_{\iota} s_{\theta}^{2} s_{\iota}^{2}+\chi_{a}^{x}\left(\frac{3}{4}+\frac{c_{2 \theta}}{4}\right) \cos (3 \alpha) s_{\iota}^{3}+\cos (2 \Psi)\left(\frac{10}{3} \chi_{a}^{x} c_{\theta} c_{\iota} s_{\theta} s_{\iota}^{2}+5 \chi_{a}^{z} c_{\iota} s_{\theta}^{2} s_{\iota}^{2}\right) \\
& +\cos (2 \alpha)\left(\chi_{a}^{z}\left(\frac{3}{2}+\frac{c_{2 \theta}}{2}\right) c_{\iota} s_{\iota}^{2}-\chi_{a}^{x} c_{\iota} s_{2 \theta} s_{\iota}^{2}\right)+\cos (\alpha)\left(\chi_{a}^{x}\left(-\frac{11}{16} c_{2 \theta} s_{\iota}-\frac{3 s_{\iota}^{3}}{4}-\frac{7}{16} c_{2 \theta} s_{3 \iota}\right)\right. \\
& \left.+\chi_{a}^{z}\left(\frac{1}{2} s_{2 \theta} s_{\iota}-\frac{1}{2} s_{2 \theta} s_{3 \iota}\right)\right)+\chi_{a}^{y}\left(\frac{15}{8}-\frac{3 c_{2 \theta}}{8}+\left(\frac{9}{8}-\frac{5 c_{2 \theta}}{8}\right) c_{2 \iota}\right) s_{\iota} \sin (\alpha)-2 \chi_{a}^{y} c_{\theta} c_{\iota} s_{\theta} s_{\iota}^{2} \sin (2 \alpha) \\
& +\chi_{a}^{y}\left(\frac{3}{4}+\frac{c_{2 \theta}}{4}\right) s_{\iota}^{3} \sin (3 \alpha)+\chi_{a}^{y} c_{\frac{\iota}{2}}\left(\frac{5}{2}-\frac{11 c_{2 \theta}}{6}+\left(\frac{15}{2}-\frac{25 c_{2 \theta}}{6}\right) c_{\iota}\right) s_{\frac{\iota}{2}}^{3} \sin (\alpha-2 \Psi) \\
& +\chi_{a}^{y}\left(-\frac{7 s_{2 \theta}}{3}-\frac{10}{3} c_{\iota} s_{2 \theta}\right) s_{\frac{\iota}{2}}^{4} \sin (2 \alpha-2 \Psi)+\chi_{a}^{y}\left(5+\frac{5 c_{2 \theta}}{3}\right) c_{\frac{\iota}{2}} s_{\frac{\iota}{2}}^{5} \sin (3 \alpha-2 \Psi)-\frac{1}{3} \chi_{a}^{y} c_{\theta} s_{\theta} s_{\iota}^{2} \sin (2 \Psi) \\
& +\chi_{a}^{y} c_{\frac{\iota}{2}}^{3}\left(\frac{5}{2}-\frac{11 c_{2 \theta}}{6}+\left(-\frac{15}{2}+\frac{25 c_{2 \theta}}{6}\right) c_{\iota}\right) s_{\frac{\iota}{2}} \sin (\alpha+2 \Psi)+\chi_{a}^{y} c_{\frac{\iota}{2}}^{4}\left(\frac{7 s_{2 \theta}}{3}-\frac{10}{3} c_{\iota} s_{2 \theta}\right) \sin (2 \alpha+2 \Psi) \\
& \left.+\chi_{a}^{y}\left(5+\frac{5 c_{2 \theta}}{3}\right) c_{\frac{l}{2}}^{5} s_{\frac{\iota}{2}} \sin (3 \alpha+2 \Psi)\right],
\end{aligned}
$$

$$
\begin{aligned}
\hat{h}_{\times}^{(0)} & =4 c_{\frac{\iota}{2}} s_{\theta} s_{\frac{\iota}{2}}^{3} \sin (\alpha-2 \Psi)-2 c_{\theta} s_{\frac{\iota}{2}}^{4} \sin (2 \alpha-2 \Psi)-4 c_{\frac{\iota}{2}}^{3} s_{\theta} s_{\frac{\iota}{2}} \sin (\alpha+2 \Psi)-2 c_{\theta} c_{\frac{\iota}{2}}^{4} \sin (2 \alpha+2 \Psi), \\
\hat{h}_{\times}^{(1 / 2)} & =\delta\left[-\frac{45}{8} c_{\frac{\iota}{2}}^{2} s_{2 \theta} s_{\frac{\iota}{2}}^{4} \sin (\alpha-3 \Psi)+\frac{9}{2} c_{2 \theta} c_{\frac{\iota}{2}} s_{\frac{\iota}{2}}^{5} \sin (2 \alpha-3 \Psi)+\frac{9}{8} s_{2 \theta} s_{\frac{\iota}{2}}^{6} \sin (3 \alpha-3 \Psi)\right. \\
& +\left(-\frac{1}{64} c_{\theta} s_{\theta}+\frac{43}{128} c_{\theta} c_{\iota} s_{\theta}-\frac{23}{128} c_{2 \iota} s_{2 \theta}+\frac{5}{256} c_{3 \iota} s_{2 \theta}\right) \sin (\alpha-\Psi) \\
& +\left(\left(-1-\frac{c_{2 \theta}}{4}\right) c_{\frac{\iota}{2}}+\frac{1}{4} c_{2 \theta} c_{\frac{\iota}{2}} c_{\iota}\right) s_{\frac{\iota}{2}}^{3} \sin (2 \alpha-\Psi)+\frac{1}{8} c_{\frac{\iota}{2}}^{2} s_{2 \theta} s_{\frac{\iota}{2}}^{4} \sin (3 \alpha-\Psi)+\frac{1}{2} s_{\theta}^{2} s_{2 \iota} \sin (\Psi)
\end{aligned}
$$




$$
\begin{aligned}
& +\left(\frac{c_{\theta} s_{\theta}}{64}+\frac{43}{128} c_{\theta} c_{\iota} s_{\theta}+\frac{23}{128} c_{2 \iota} s_{2 \theta}+\frac{5}{256} c_{3 \iota} s_{2 \theta}\right) \sin (\alpha+\Psi)+\left(\left(-1-\frac{c_{2 \theta}}{4}\right) c_{\frac{\iota}{2}}^{3}-\frac{1}{4} c_{2 \theta} c_{\frac{\iota}{2}}^{3} c_{\iota}\right) s_{\frac{\iota}{2}} \sin (2 \alpha+\Psi) \\
& \left.-\frac{1}{8} c_{\frac{\iota}{2}}^{4} s_{2 \theta} s_{\frac{\iota}{2}}^{2} \sin (3 \alpha+\Psi)+\frac{45}{8} c_{\frac{\iota}{2}}^{4} s_{2 \theta} s_{\frac{\iota}{2}}^{2} \sin (\alpha+3 \Psi)+\frac{9}{2} c_{2 \theta} c_{\frac{\iota}{2}}^{5} s_{\frac{\iota}{2}} \sin (2 \alpha+3 \Psi)-\frac{9}{8} c_{\frac{\iota}{2}}^{6} s_{2 \theta} \sin (3 \alpha+3 \Psi)\right] \text {, } \\
& \hat{h}_{\times}^{(1)}=\left(\nu c_{\frac{\iota}{2}}^{3}\left(-12 s_{\theta}-28 s_{3 \theta}\right) s_{\frac{L}{2}}^{5}+c_{\frac{\iota}{2}}^{3}\left(4 s_{\theta}+\frac{28 s_{3 \theta}}{3}\right) s_{\frac{L}{2}}^{5}\right) \sin (\alpha-4 \Psi)+\left(\left(-\frac{4 c_{\theta}}{3}-\frac{28 c_{3 \theta}}{3}\right) c_{\frac{\iota}{2}}^{2} s_{\frac{L}{2}}^{6}\right. \\
& \left.+\nu\left(4 c_{\theta}+28 c_{3 \theta}\right) c_{\frac{\iota}{2}}^{2} s_{\frac{\iota}{2}}^{6}\right) \sin (2 \alpha-4 \Psi)+\left(c_{\frac{\iota}{2}}\left(\frac{4 s_{\theta}}{3}-4 s_{3 \theta}\right) s_{\frac{\iota}{2}}^{7}+\nu c_{\frac{\iota}{2}}\left(-4 s_{\theta}+12 s_{3 \theta}\right) s_{\frac{\iota}{2}}^{7}\right) \sin (3 \alpha-4 \Psi) \\
& +\left(-\frac{8}{3} c_{\theta} s_{\theta}^{2} s_{\frac{\iota}{2}}^{8}+8 \nu c_{\theta} s_{\theta}^{2} s_{\frac{\iota}{2}}^{8}\right) \sin (4 \alpha-4 \Psi)+\left[c _ { \frac { \iota } { 2 } } \left(-\frac{79 s_{\theta}}{8}+c_{\iota}\left(\frac{3 s_{\theta}}{4}-\frac{19 s_{3 \theta}}{12}\right)+c_{2 \iota}\left(\frac{s_{\theta}}{8}+\frac{7 s_{3 \theta}}{24}\right)\right.\right. \\
& \left.\left.-\frac{3 s_{3 \theta}}{8}\right) s_{\frac{\iota}{2}}^{3}+\nu c_{\frac{\iota}{2}}\left(\frac{103 s_{\theta}}{24}+c_{2 \iota}\left(-\frac{3 s_{\theta}}{8}-\frac{7 s_{3 \theta}}{8}\right)+\frac{9 s_{3 \theta}}{8}+c_{\iota}\left(-\frac{9 s_{\theta}}{4}+\frac{19 s_{3 \theta}}{4}\right)\right) s_{\frac{\iota}{2}}^{3}\right] \sin (\alpha-2 \Psi) \\
& +\left[\left(\frac{47 c_{\theta}}{8}+\frac{c_{3 \theta}}{8}+\left(\frac{7 c_{\theta}}{6}+\frac{c_{3 \theta}}{6}\right) c_{\iota}+\left(-\frac{c_{\theta}}{24}-\frac{7 c_{3 \theta}}{24}\right) c_{2 \iota}\right) s_{\frac{\iota}{2}}^{4}+\nu\left(-\frac{119 c_{\theta}}{24}-\frac{3 c_{3 \theta}}{8}+\left(-\frac{7 c_{\theta}}{2}-\frac{c_{3 \theta}}{2}\right) c_{\iota}\right.\right. \\
& \left.\left.+\left(\frac{c_{\theta}}{8}+\frac{7 c_{3 \theta}}{8}\right) c_{2 \iota}\right) s_{\frac{\iota}{2}}^{4}\right] \sin (2 \alpha-2 \Psi)+\left[c_{\frac{\iota}{2}}\left(\frac{4 s_{\theta}}{3}+\left(-\frac{1}{3}-c_{2 \theta}\right) c_{\iota} s_{\theta}\right) s_{\frac{\iota}{2}}^{5}\right. \\
& \left.+\nu c_{\frac{\iota}{2}}\left(-4 s_{\theta}+\left(1+3 c_{2 \theta}\right) c_{\iota} s_{\theta}\right) s_{\frac{\iota}{2}}^{5}\right] \sin (3 \alpha-2 \Psi)+\left(-\frac{2}{3} c_{\theta} c_{\frac{\iota}{2}}^{2} s_{\theta}^{2} s_{\frac{\iota}{2}}^{6}+2 \nu c_{\theta} c_{\frac{\iota}{2}}^{2} s_{\theta}^{2} s_{\frac{\iota}{2}}^{6}\right) \sin (4 \alpha-2 \Psi) \\
& +\left(-\frac{5}{2} c_{\theta} c_{\iota} s_{\theta}^{2} s_{\iota}^{2}+\frac{15}{2} \nu c_{\theta} c_{\iota} s_{\theta}^{2} s_{\iota}^{2}\right) \sin (2 \Psi)+\left[c _ { \frac { \iota } { 2 } } ^ { 3 } \left(\frac{79 s_{\theta}}{8}+c_{\iota}\left(\frac{3 s_{\theta}}{4}-\frac{19 s_{3 \theta}}{12}\right)+c_{2 \iota}\left(-\frac{s_{\theta}}{8}-\frac{7 s_{3 \theta}}{24}\right)\right.\right. \\
& \left.\left.+\frac{3 s_{3 \theta}}{8}\right) s_{\frac{\iota}{2}}+\nu c_{\frac{\iota}{2}}^{3}\left(-\frac{103 s_{\theta}}{24}+c_{2 \iota}\left(\frac{3 s_{\theta}}{8}+\frac{7 s_{3 \theta}}{8}\right)-\frac{9 s_{3 \theta}}{8}+c_{\iota}\left(-\frac{9 s_{\theta}}{4}+\frac{19 s_{3 \theta}}{4}\right)\right) s_{\frac{\iota}{2}}\right] \sin (\alpha+2 \Psi) \\
& +\left[c_{\frac{\iota}{2}}^{4}\left(\frac{47 c_{\theta}}{8}+\frac{c_{3 \theta}}{8}+\left(-\frac{7 c_{\theta}}{6}-\frac{c_{3 \theta}}{6}\right) c_{\iota}+\left(-\frac{c_{\theta}}{24}-\frac{7 c_{3 \theta}}{24}\right) c_{2 \iota}\right)+\nu c_{\frac{\iota}{2}}^{4}\left(-\frac{119 c_{\theta}}{24}-\frac{3 c_{3 \theta}}{8}+\left(\frac{7 c_{\theta}}{2}+\frac{c_{3 \theta}}{2}\right) c_{\iota}\right.\right. \\
& \left.\left.+\left(\frac{c_{\theta}}{8}+\frac{7 c_{3 \theta}}{8}\right) c_{2 \iota}\right)\right] \sin (2 \alpha+2 \Psi)+\left[c_{\frac{\iota}{2}}^{5}\left(-\frac{4 s_{\theta}}{3}+\left(-\frac{1}{3}-c_{2 \theta}\right) c_{\iota} s_{\theta}\right) s_{\frac{\iota}{2}}\right. \\
& \left.+\nu c_{\frac{\iota}{2}}^{5}\left(4 s_{\theta}+\left(1+3 c_{2 \theta}\right) c_{\iota} s_{\theta}\right) s_{\frac{\iota}{2}}\right] \sin (3 \alpha+2 \Psi)+\left(-\frac{2}{3} c_{\theta} c_{\frac{\iota}{2}}^{6} s_{\theta}^{2} s_{\frac{\iota}{2}}^{2}+2 \nu c_{\theta} c_{\frac{\iota}{2}}^{6} s_{\theta}^{2} s_{\frac{\iota}{2}}^{2}\right) \sin (4 \alpha+2 \Psi) \\
& +\left(c_{\frac{\iota}{2}}^{5}\left(-4 s_{\theta}-\frac{28 s_{3 \theta}}{3}\right) s_{\frac{\iota}{2}}^{3}+\nu c_{\frac{\iota}{2}}^{5}\left(12 s_{\theta}+28 s_{3 \theta}\right) s_{\frac{\iota}{2}}^{3}\right) \sin (\alpha+4 \Psi)+\left(\left(-\frac{4 c_{\theta}}{3}-\frac{28 c_{3 \theta}}{3}\right) c_{\frac{\iota}{2}}^{6} s_{\frac{\iota}{2}}^{2}\right. \\
& \left.+\nu\left(4 c_{\theta}+28 c_{3 \theta}\right) c_{\frac{\iota}{2}}^{6} s_{\frac{\iota}{2}}^{2}\right) \sin (2 \alpha+4 \Psi)+\left(\nu\left(-8-24 c_{2 \theta}\right) c_{\frac{\iota}{2}}^{7} s_{\theta} s_{\frac{\iota}{2}}+\left(\frac{8}{3}+8 c_{2 \theta}\right) c_{\frac{\iota}{2}}^{7} s_{\theta} s_{\frac{\iota}{2}}\right) \sin (3 \alpha+4 \Psi) \\
& +\left(-\frac{8}{3} c_{\theta} c_{\frac{\iota}{2}}^{8} s_{\theta}^{2}+8 \nu c_{\theta} c_{\frac{L}{2}}^{8} s_{\theta}^{2}\right) \sin (4 \alpha+4 \Psi), \\
& \hat{h}_{\times}^{(3 / 2)}=8 \pi c_{\frac{\iota}{2}} s_{\theta} s_{\frac{\iota}{2}}^{3} \sin (\alpha-2 \Psi)-4 \pi c_{\theta} s_{\frac{\iota}{2}}^{4} \sin (2 \alpha-2 \Psi)-8 \pi c_{\frac{\iota}{2}}^{3} s_{\theta} s_{\frac{\iota}{2}} \sin (\alpha+2 \Psi)-4 \pi c_{\theta} c_{\frac{\iota}{2}}^{4} \sin (2 \alpha+2 \Psi) \\
& +\delta\left[\left(c_{\frac{L}{2}}^{4}\left(-\frac{4375 s_{2 \theta}}{384}-\frac{4375 s_{4 \theta}}{256}\right) s_{\frac{L}{2}}^{6}+\nu c_{\frac{\iota}{2}}^{4}\left(\frac{4375 s_{2 \theta}}{192}+\frac{4375 s_{4 \theta}}{128}\right) s_{\frac{L}{2}}^{6}\right) \sin (\alpha-5 \Psi)\right. \\
& +\left(\nu\left(-\frac{625 c_{2 \theta}}{48}-\frac{625 c_{4 \theta}}{16}\right) c_{\frac{L}{2}}^{3} s_{\frac{L}{2}}^{7}+\left(\frac{625 c_{2 \theta}}{96}+\frac{625 c_{4 \theta}}{32}\right) c_{\frac{L}{2}}^{3} s_{\frac{L}{2}}^{7}\right) \sin (2 \alpha-5 \Psi) \\
& +\left(\nu c_{\frac{\iota}{2}}^{2}\left(\frac{625 s_{2 \theta}}{128}-\frac{5625 s_{4 \theta}}{256}\right) s_{\frac{\iota}{2}}^{8}+c_{\frac{\iota}{2}}^{2}\left(-\frac{625 s_{2 \theta}}{256}+\frac{5625 s_{4 \theta}}{512}\right) s_{\frac{\iota}{2}}^{8}\right) \sin (3 \alpha-5 \Psi) \\
& +\left(\nu\left(-\frac{625}{48}-\frac{625 c_{2 \theta}}{24}\right) c_{\frac{\iota}{2}} s_{\theta}^{2} s_{\frac{\iota}{2}}^{9}+\left(\frac{625}{96}+\frac{625 c_{2 \theta}}{48}\right) c_{\frac{\iota}{2}} s_{\theta}^{2} s_{\frac{\iota}{2}}^{9}\right) \sin (4 \alpha-5 \Psi) \\
& +\left(\frac{625}{192} c_{\theta} s_{\theta}^{3} s_{\frac{\iota}{2}}^{10}-\frac{625}{96} \nu c_{\theta} s_{\theta}^{3} s_{\frac{\iota}{2}}^{10}\right) \sin (5 \alpha-5 \Psi)+\left[\nu c _ { \frac { \iota } { 2 } } ^ { 2 } \left(-\frac{4923 s_{2 \theta}}{512}+c_{\iota}\left(\frac{459 s_{2 \theta}}{128}-\frac{2079 s_{4 \theta}}{256}\right)-\frac{945 s_{4 \theta}}{1024}\right.\right. \\
& \left.+c_{2 \iota}\left(\frac{567 s_{2 \theta}}{512}+\frac{1701 s_{4 \theta}}{1024}\right)\right) s_{\frac{\iota}{2}}^{4}+c_{\frac{\iota}{2}}^{2}\left(\frac{22203 s_{2 \theta}}{1024}+c_{2 \iota}\left(-\frac{567 s_{2 \theta}}{1024}-\frac{1701 s_{4 \theta}}{2048}\right)+\frac{945 s_{4 \theta}}{2048}\right.
\end{aligned}
$$




$$
\begin{aligned}
& \left.\left.+c_{\iota}\left(-\frac{459 s_{2 \theta}}{256}+\frac{2079 s_{4 \theta}}{512}\right)\right) s_{\frac{\iota}{2}}^{4}\right] \sin (\alpha-3 \Psi)+\left[\nu c _ { \frac { \iota } { 2 } } \left(\frac{27}{16}+\frac{1233 c_{2 \theta}}{128}+\frac{27 c_{4 \theta}}{128}+\left(\frac{27}{8}+\frac{27 c_{2 \theta}}{16}+\frac{27 c_{4 \theta}}{16}\right) c_{\iota}\right.\right. \\
& \left.+\left(-\frac{81 c_{2 \theta}}{128}-\frac{243 c_{4 \theta}}{128}\right) c_{2 \iota}\right) s_{\frac{\iota}{2}}^{5}+c_{\frac{\iota}{2}}\left(-\frac{27}{32}-\frac{4689 c_{2 \theta}}{256}-\frac{27 c_{4 \theta}}{256}+\left(-\frac{27}{16}-\frac{27 c_{2 \theta}}{32}-\frac{27 c_{4 \theta}}{32}\right) c_{\iota}\right. \\
& \left.\left.+\left(\frac{81 c_{2 \theta}}{256}+\frac{243 c_{4 \theta}}{256}\right) c_{2 \iota}\right) s_{\frac{\iota}{2}}^{5}\right] \sin (2 \alpha-3 \Psi)+\left[\nu \left(\left(\frac{4761}{1024}-\frac{1377 c_{2 \theta}}{1024}\right) s_{2 \theta}+\left(\frac{837}{256}-\frac{621 c_{2 \theta}}{256}\right) c_{\iota} s_{2 \theta}\right.\right. \\
& \left.+\left(\frac{243}{1024}-\frac{2187 c_{2 \theta}}{1024}\right) c_{2 \iota} s_{2 \theta}\right) s_{\frac{\iota}{2}}^{6}+\left(\left(-\frac{11673}{2048}+\frac{1377 c_{2 \theta}}{2048}\right) s_{2 \theta}+\left(-\frac{837}{512}+\frac{621 c_{2 \theta}}{512}\right) c_{\iota} s_{2 \theta}\right. \\
& \left.\left.+\left(-\frac{243}{2048}+\frac{2187 c_{2 \theta}}{2048}\right) c_{2 \iota} s_{2 \theta}\right) s_{\frac{\iota}{2}}^{6}\right] \sin (3 \alpha-3 \Psi)+\left[\nu c_{\frac{\iota}{2}}\left(\left(\frac{81}{32}-\frac{27 c_{2 \theta}}{16}\right) s_{\theta}^{2}+\left(-\frac{81}{32}-\frac{81 c_{2 \theta}}{16}\right) c_{\iota} s_{\theta}^{2}\right) s_{\frac{\iota}{2}}^{7}\right. \\
& \left.+c_{\frac{\iota}{2}}\left(\left(-\frac{81}{64}+\frac{27 c_{2 \theta}}{32}\right) s_{\theta}^{2}+\left(\frac{81}{64}+\frac{81 c_{2 \theta}}{32}\right) c_{\iota} s_{\theta}^{2}\right) s_{\frac{L}{2}}^{7}\right] \sin (4 \alpha-3 \Psi) \\
& +\left(\frac{81}{64} c_{\theta} c_{\frac{\iota}{2}}^{2} s_{\theta}^{3} s_{\frac{\iota}{2}}^{8}-\frac{81}{32} \nu c_{\theta} c_{\frac{\iota}{2}}^{2} s_{\theta}^{3} s_{\frac{\iota}{2}}^{8}\right) \sin (5 \alpha-3 \Psi)+\left[\frac{683 c_{\theta} s_{\theta}}{16384}+\left(\frac{557}{4096}-\frac{11 c_{2 \theta}}{12288}\right) c_{2 \iota} s_{2 \theta}\right. \\
& +\left(-\frac{1719}{32768}+\frac{91 c_{2 \theta}}{32768}\right) c_{3 \iota} s_{2 \theta}-\frac{c_{\theta} s_{3 \theta}}{16384}+c_{\iota}\left(-\frac{10511 c_{\theta} s_{\theta}}{49152}+\frac{173 c_{\theta} s_{3 \theta}}{49152}\right)+\nu\left(\frac{85 c_{\theta} s_{\theta}}{8192}\right. \\
& +\left(-\frac{679}{6144}+\frac{11 c_{2 \theta}}{6144}\right) c_{2 \iota} s_{2 \theta}+\left(-\frac{201}{16384}-\frac{91 c_{2 \theta}}{16384}\right) c_{3 \iota} s_{2 \theta}+\frac{c_{\theta} s_{3 \theta}}{8192}+c_{\iota}\left(\frac{6031 c_{\theta} s_{\theta}}{24576}-\frac{173 c_{\theta} s_{3 \theta}}{24576}\right) \\
& \left.+c_{5 \iota}\left(-\frac{7 s_{2 \theta}}{49152}-\frac{7 s_{4 \theta}}{32768}\right)+c_{4 \iota}\left(-\frac{37 s_{2 \theta}}{24576}+\frac{91 s_{4 \theta}}{16384}\right)\right)+c_{4 \iota}\left(\frac{37 s_{2 \theta}}{49152}-\frac{91 s_{4 \theta}}{32768}\right) \\
& \left.+c_{5 \iota}\left(\frac{7 s_{2 \theta}}{98304}+\frac{7 s_{4 \theta}}{65536}\right)\right] \sin (\alpha-\Psi)+\left[\nu \left(\frac{19}{512} c_{4 \theta} c_{\frac{3 \iota}{2}}+\frac{9}{512} c_{4 \theta} c_{\frac{5 \iota}{2}}+c_{\frac{\iota}{2}}\left(-\frac{11}{16}-\frac{35 c_{2 \theta}}{128}+\frac{79 c_{4 \theta}}{1536}\right.\right.\right. \\
& \left.\left.+\left(\frac{1}{32}-\frac{37 c_{2 \theta}}{256}\right) c_{\iota}+\left(\frac{1}{32}+\frac{3 c_{2 \theta}}{128}\right) c_{2 \iota}-\frac{1}{768} c_{2 \theta} c_{3 \iota}\right)-\frac{1}{512} c_{4 \theta} c_{\frac{7 \iota}{2}}\right) s_{\frac{\iota}{2}}^{3}+\left(-\frac{19 c_{4 \theta} c_{\frac{3 \iota}{2}}}{1024}-\frac{9 c_{4 \theta} c_{\frac{5 \iota}{2}}}{1024}\right. \\
& +c_{\frac{\iota}{2}}\left(\frac{19}{32}-\frac{23 c_{2 \theta}}{768}-\frac{79 c_{4 \theta}}{3072}+\left(-\frac{1}{64}-\frac{347 c_{2 \theta}}{512}\right) c_{\iota}+\left(-\frac{1}{64}-\frac{3 c_{2 \theta}}{256}\right) c_{2 \iota}+\frac{c_{2 \theta} c_{3 \iota}}{1536}\right) \\
& \left.\left.+\frac{c_{4 \theta} c_{\frac{7 \iota}{2}}}{1024}\right) s_{\frac{\iota}{2}}^{3}\right] \sin (2 \alpha-\Psi)+\left[c_{\frac{\iota}{2}}^{2}\left(-\frac{355 s_{2 \theta}}{1024}+c_{\iota}\left(-\frac{13 s_{2 \theta}}{256}-\frac{11 s_{4 \theta}}{512}\right)+c_{2 \iota}\left(-\frac{s_{2 \theta}}{1024}+\frac{9 s_{4 \theta}}{2048}\right)-\frac{5 s_{4 \theta}}{2048}\right) s_{\frac{\iota}{2}}^{4}\right. \\
& \left.+\nu c_{\frac{\iota}{2}}^{2}\left(-\frac{29 s_{2 \theta}}{512}+c_{2 \iota}\left(\frac{s_{2 \theta}}{512}-\frac{9 s_{4 \theta}}{1024}\right)+c_{\iota}\left(\frac{13 s_{2 \theta}}{128}+\frac{11 s_{4 \theta}}{256}\right)+\frac{5 s_{4 \theta}}{1024}\right) s_{\frac{\iota}{2}}^{4}\right] \sin (3 \alpha-\Psi) \\
& +\left[\nu c_{\frac{\iota}{2}}^{3}\left(\left(\frac{7}{48}+\frac{c_{2 \theta}}{24}\right) s_{\theta}^{2}+\left(-\frac{1}{48}-\frac{c_{2 \theta}}{24}\right) c_{\iota} s_{\theta}^{2}\right) s_{\frac{L}{2}}^{5}+c_{\frac{\iota}{2}}^{3}\left(\left(-\frac{7}{96}-\frac{c_{2 \theta}}{48}\right) s_{\theta}^{2}\right.\right. \\
& \left.\left.+\left(\frac{1}{96}+\frac{c_{2 \theta}}{48}\right) c_{\iota} s_{\theta}^{2}\right) s_{\frac{\iota}{2}}^{5}\right] \sin (4 \alpha-\Psi)+\left(\frac{1}{96} c_{\theta} c_{\frac{\iota}{2}}^{4} s_{\theta}^{3} s_{\frac{\iota}{2}}^{6}-\frac{1}{48} \nu c_{\theta} c_{\frac{\iota}{2}}^{4} s_{\theta}^{3} s_{\frac{\iota}{2}}^{6}\right) \sin (5 \alpha-\Psi) \\
& +\left[\left(-\frac{77}{256}+\frac{c_{2 \theta}}{256}\right) s_{\theta}^{2} s_{2 \iota}+\left(\frac{5}{512}+\frac{7 c_{2 \theta}}{512}\right) s_{\theta}^{2} s_{4 \iota}+\nu\left(\left(\frac{45}{128}-\frac{c_{2 \theta}}{128}\right) s_{\theta}^{2} s_{2 \iota}+\left(-\frac{5}{256}-\frac{7 c_{2 \theta}}{256}\right) s_{\theta}^{2} s_{4 \iota}\right)\right] \sin (\Psi) \\
& +\left(\nu\left(-\frac{135}{32}-\frac{189 c_{2 \theta}}{32}\right) c_{\iota} s_{\theta}^{2} s_{\iota}^{3}+\left(\frac{135}{64}+\frac{189 c_{2 \theta}}{64}\right) c_{\iota} s_{\theta}^{2} s_{\iota}^{3}\right) \sin (3 \Psi)+\left[-\frac{683 c_{\theta} s_{\theta}}{16384}\right. \\
& +\left(-\frac{557}{4096}+\frac{11 c_{2 \theta}}{12288}\right) c_{2 \iota} s_{2 \theta}+\left(-\frac{1719}{32768}+\frac{91 c_{2 \theta}}{32768}\right) c_{3 \iota} s_{2 \theta}+\frac{c_{\theta} s_{3 \theta}}{16384}+c_{\iota}\left(-\frac{10511 c_{\theta} s_{\theta}}{49152}+\frac{173 c_{\theta} s_{3 \theta}}{49152}\right) \\
& +\nu\left(-\frac{85 c_{\theta} s_{\theta}}{8192}+\left(\frac{679}{6144}-\frac{11 c_{2 \theta}}{6144}\right) c_{2 \iota} s_{2 \theta}+\left(-\frac{201}{16384}-\frac{91 c_{2 \theta}}{16384}\right) c_{3 \iota} s_{2 \theta}-\frac{c_{\theta} s_{3 \theta}}{8192}+c_{\iota}\left(\frac{6031 c_{\theta} s_{\theta}}{24576}-\frac{173 c_{\theta} s_{3 \theta}}{24576}\right)\right. \\
& \left.+c_{4 \iota}\left(\frac{37 s_{2 \theta}}{24576}-\frac{91 s_{4 \theta}}{16384}\right)+c_{5 \iota}\left(-\frac{7 s_{2 \theta}}{49152}-\frac{7 s_{4 \theta}}{32768}\right)\right)+c_{5 \iota}\left(\frac{7 s_{2 \theta}}{98304}+\frac{7 s_{4 \theta}}{65536}\right) \\
& \left.+c_{4 \iota}\left(-\frac{37 s_{2 \theta}}{49152}+\frac{91 s_{4 \theta}}{32768}\right)\right] \sin (\alpha+\Psi)+\left[c _ { \frac { \iota } { 2 } } ^ { 3 } \left(\frac{19}{32}-\frac{23 c_{2 \theta}}{768}-\frac{79 c_{4 \theta}}{3072}+\left(\frac{1}{64}+\frac{347 c_{2 \theta}}{512}\right) c_{\iota}\right.\right. \\
& \left.+\left(-\frac{1}{64}-\frac{3 c_{2 \theta}}{256}\right) c_{2 \iota}-\frac{c_{2 \theta} c_{3 \iota}}{1536}\right) s_{\frac{\iota}{2}}+\frac{19 c_{4 \theta} c_{\frac{\iota}{2}}^{3} s_{\frac{3 \iota}{2}}}{1024}-\frac{9 c_{4 \theta} c_{\frac{\iota}{2}}^{3} s_{\frac{5 \iota}{2}}}{1024}-\frac{c_{4 \theta} c_{\frac{\iota}{2}}^{3} s_{\frac{7 \iota}{2}}}{1024}+\nu\left(c _ { \frac { \iota } { 2 } } ^ { 3 } \left(-\frac{11}{16}-\frac{35 c_{2 \theta}}{128}+\frac{79 c_{4 \theta}}{1536}\right.\right.
\end{aligned}
$$




$$
\begin{aligned}
& \left.+\left(-\frac{1}{32}+\frac{37 c_{2 \theta}}{256}\right) c_{\iota}+\left(\frac{1}{32}+\frac{3 c_{2 \theta}}{128}\right) c_{2 \iota}+\frac{1}{768} c_{2 \theta} c_{3 \iota}\right) s_{\frac{\iota}{2}}-\frac{19}{512} c_{4 \theta} c_{\frac{\iota}{2}}^{3} s_{\frac{3 \iota}{2}}+\frac{9}{512} c_{4 \theta} c_{\frac{\iota}{2}}^{3} s_{\frac{5 \iota}{2}} \\
& \left.\left.+\frac{1}{512} c_{4 \theta} c_{\frac{\iota}{2}}^{3} s_{\frac{7 \iota}{2}}\right)\right] \sin (2 \alpha+\Psi)+\left[\nu c_{\frac{\iota}{2}}^{4}\left(\frac{29 s_{2 \theta}}{512}+c_{2 \iota}\left(-\frac{s_{2 \theta}}{512}+\frac{9 s_{4 \theta}}{1024}\right)+c_{\iota}\left(\frac{13 s_{2 \theta}}{128}+\frac{11 s_{4 \theta}}{256}\right)-\frac{5 s_{4 \theta}}{1024}\right) s_{\frac{\iota}{2}}^{2}\right. \\
& \left.+c_{\frac{\iota}{2}}^{4}\left(\frac{355 s_{2 \theta}}{1024}+c_{\iota}\left(-\frac{13 s_{2 \theta}}{256}-\frac{11 s_{4 \theta}}{512}\right)+c_{2 \iota}\left(\frac{s_{2 \theta}}{1024}-\frac{9 s_{4 \theta}}{2048}\right)+\frac{5 s_{4 \theta}}{2048}\right) s_{\frac{\iota}{2}}^{2}\right] \sin (3 \alpha+\Psi) \\
& +\left[c_{\frac{\iota}{2}}^{5}\left(\left(-\frac{7}{96}-\frac{c_{2 \theta}}{48}\right) s_{\theta}^{2}+\left(-\frac{1}{96}-\frac{c_{2 \theta}}{48}\right) c_{\iota} s_{\theta}^{2}\right) s_{\frac{\iota}{2}}^{3}+\nu c_{\frac{\iota}{2}}^{5}\left(\left(\frac{7}{48}+\frac{c_{2 \theta}}{24}\right) s_{\theta}^{2}\right.\right. \\
& \left.\left.+\left(\frac{1}{48}+\frac{c_{2 \theta}}{24}\right) c_{\iota} s_{\theta}^{2}\right) s_{\frac{\iota}{2}}^{3}\right] \sin (4 \alpha+\Psi)+\left(-\frac{1}{96} c_{\theta} c_{\frac{\iota}{2}}^{6} s_{\theta}^{3} s_{\frac{\iota}{2}}^{4}+\frac{1}{48} \nu c_{\theta} c_{\frac{L}{2}}^{6} s_{\theta}^{3} s_{\frac{\iota}{2}}^{4}\right) \sin (5 \alpha+\Psi) \\
& +\left[\nu c_{\frac{\iota}{2}}^{4}\left(\frac{4923 s_{2 \theta}}{512}+c_{\iota}\left(\frac{459 s_{2 \theta}}{128}-\frac{2079 s_{4 \theta}}{256}\right)+c_{2 \iota}\left(-\frac{567 s_{2 \theta}}{512}-\frac{1701 s_{4 \theta}}{1024}\right)+\frac{945 s_{4 \theta}}{1024}\right) s_{\frac{\iota}{2}}^{2}+c_{\frac{\iota}{2}}^{4}\left(-\frac{22203 s_{2 \theta}}{1024}\right.\right. \\
& \left.\left.+c_{2 \iota}\left(\frac{567 s_{2 \theta}}{1024}+\frac{1701 s_{4 \theta}}{2048}\right)-\frac{945 s_{4 \theta}}{2048}+c_{\iota}\left(-\frac{459 s_{2 \theta}}{256}+\frac{2079 s_{4 \theta}}{512}\right)\right) s_{\frac{\iota}{2}}^{2}\right] \sin (\alpha+3 \Psi) \\
& +\left[\nu c_{\frac{\iota}{2}}^{5}\left(\frac{27}{16}+\frac{1233 c_{2 \theta}}{128}+\frac{27 c_{4 \theta}}{128}+\left(-\frac{27}{8}-\frac{27 c_{2 \theta}}{16}-\frac{27 c_{4 \theta}}{16}\right) c_{\iota}+\left(-\frac{81 c_{2 \theta}}{128}-\frac{243 c_{4 \theta}}{128}\right) c_{2 \iota}\right) s_{\frac{\iota}{2}}\right. \\
& \left.+c_{\frac{\iota}{2}}^{5}\left(-\frac{27}{32}-\frac{4689 c_{2 \theta}}{256}-\frac{27 c_{4 \theta}}{256}+\left(\frac{27}{16}+\frac{27 c_{2 \theta}}{32}+\frac{27 c_{4 \theta}}{32}\right) c_{\iota}+\left(\frac{81 c_{2 \theta}}{256}+\frac{243 c_{4 \theta}}{256}\right) c_{2 \iota}\right) s_{\frac{\iota}{2}}\right] \sin (2 \alpha+3 \Psi) \\
& +\left[c_{\frac{\iota}{2}}^{6}\left(\frac{11673 s_{2 \theta}}{2048}+c_{2 \iota}\left(\frac{243 s_{2 \theta}}{2048}-\frac{2187 s_{4 \theta}}{4096}\right)+c_{\iota}\left(-\frac{837 s_{2 \theta}}{512}+\frac{621 s_{4 \theta}}{1024}\right)-\frac{1377 s_{4 \theta}}{4096}\right)+\nu c_{\frac{\iota}{2}}^{6}\left(-\frac{4761 s_{2 \theta}}{1024}\right.\right. \\
& \left.\left.+c_{\iota}\left(\frac{837 s_{2 \theta}}{256}-\frac{621 s_{4 \theta}}{512}\right)+\frac{1377 s_{4 \theta}}{2048}+c_{2 \iota}\left(-\frac{243 s_{2 \theta}}{1024}+\frac{2187 s_{4 \theta}}{2048}\right)\right)\right] \sin (3 \alpha+3 \Psi) \\
& +\left[c_{\frac{\iota}{2}}^{7}\left(\left(-\frac{81}{64}+\frac{27 c_{2 \theta}}{32}\right) s_{\theta}^{2}+\left(-\frac{81}{64}-\frac{81 c_{2 \theta}}{32}\right) c_{\iota} s_{\theta}^{2}\right) s_{\frac{\iota}{2}}+\nu c_{\frac{\iota}{2}}^{7}\left(\left(\frac{81}{32}-\frac{27 c_{2 \theta}}{16}\right) s_{\theta}^{2}\right.\right. \\
& \left.\left.+\left(\frac{81}{32}+\frac{81 c_{2 \theta}}{16}\right) c_{\iota} s_{\theta}^{2}\right) s_{\frac{\iota}{2}}\right] \sin (4 \alpha+3 \Psi)+\left(-\frac{81}{64} c_{\theta} c_{\frac{\iota}{2}}^{8} s_{\theta}^{3} s_{\frac{\iota}{2}}^{2}+\frac{81}{32} \nu c_{\theta} c_{\frac{\iota}{2}}^{8} s_{\theta}^{3} s_{\frac{\iota}{2}}^{2}\right) \sin (5 \alpha+3 \Psi) \\
& +\left(\nu c_{\frac{L}{2}}^{6}\left(-\frac{4375 s_{2 \theta}}{192}-\frac{4375 s_{4 \theta}}{128}\right) s_{\frac{L}{2}}^{4}+c_{\frac{L}{2}}^{6}\left(\frac{4375 s_{2 \theta}}{384}+\frac{4375 s_{4 \theta}}{256}\right) s_{\frac{L}{2}}^{4}\right) \sin (\alpha+5 \Psi) \\
& +\left(\nu\left(-\frac{625 c_{2 \theta}}{48}-\frac{625 c_{4 \theta}}{16}\right) c_{\frac{L}{2}}^{7} s_{\frac{L}{2}}^{3}+\left(\frac{625 c_{2 \theta}}{96}+\frac{625 c_{4 \theta}}{32}\right) c_{\frac{L}{2}}^{7} s_{\frac{L}{2}}^{3}\right) \sin (2 \alpha+5 \Psi)+\left(c_{\frac{L}{2}}^{8}\left(\frac{625 s_{2 \theta}}{256}-\frac{5625 s_{4 \theta}}{512}\right) s_{\frac{L}{2}}^{2}\right. \\
& \left.+\nu c_{\frac{\iota}{2}}^{8}\left(-\frac{625 s_{2 \theta}}{128}+\frac{5625 s_{4 \theta}}{256}\right) s_{\frac{\iota}{2}}^{2}\right) \sin (3 \alpha+5 \Psi)+\left(\nu\left(-\frac{625}{48}-\frac{625 c_{2 \theta}}{24}\right) c_{\frac{\iota}{2}}^{9} s_{\theta}^{2} s_{\frac{\iota}{2}}\right. \\
& \left.\left.+\left(\frac{625}{96}+\frac{625 c_{2 \theta}}{48}\right) c_{\frac{\iota}{2}}^{9} s_{\theta}^{2} s_{\frac{\iota}{2}}\right) \sin (4 \alpha+5 \Psi)+\left(-\frac{625}{192} c_{\theta} c_{\frac{\iota}{2}}^{10} s_{\theta}^{3}+\frac{625}{96} \nu c_{\theta} c_{\frac{\iota}{2}}^{10} s_{\theta}^{3}\right) \sin (5 \alpha+5 \Psi)\right], \\
& \hat{h}_{\times}^{(1, \mathrm{SO})}=\chi_{a}^{y}\left(\frac{1}{2}+\frac{c_{\iota}}{2}\right) \cos (\alpha+\Psi)+\chi_{a}^{y} \cos (\alpha-\Psi) s_{\frac{\iota}{2}}^{2}+\left(\chi_{a}^{x}\left(\frac{c_{\theta}^{2}}{2}-\frac{1}{2} c_{\theta}^{2} c_{\iota}\right)+\chi_{a}^{z}\left(-\frac{1}{2} c_{\theta} s_{\theta}+\frac{1}{2} c_{\theta} c_{\iota} s_{\theta}\right)\right) \sin (\alpha-\Psi) \\
& +\left(\chi_{a}^{x} c_{\theta} s_{\theta} s_{\iota}-\chi_{a}^{z} s_{\theta}^{2} s_{\iota}\right) \sin (\Psi)+\left(\chi_{a}^{x}\left(\frac{c_{\theta}^{2}}{2}+\frac{1}{2} c_{\theta}^{2} c_{\iota}\right)+\chi_{a}^{z}\left(-\frac{1}{2} c_{\theta} s_{\theta}-\frac{1}{2} c_{\theta} c_{\iota} s_{\theta}\right)\right) \sin (\alpha+\Psi) \\
& +\delta\left[\chi_{s}^{y}\left(\frac{1}{2}+\frac{c_{\iota}}{2}\right) \cos (\alpha+\Psi)+\chi_{s}^{y} \cos (\alpha-\Psi) s_{\frac{\iota}{2}}^{2}+\left(\chi_{s}^{x}\left(\frac{c_{\theta}^{2}}{2}-\frac{1}{2} c_{\theta}^{2} c_{\iota}\right)\right.\right. \\
& \left.+\chi_{s}^{z}\left(-\frac{1}{2} c_{\theta} s_{\theta}+\frac{1}{2} c_{\theta} c_{\iota} s_{\theta}\right)\right) \sin (\alpha-\Psi)+\left(\chi_{s}^{x} c_{\theta} s_{\theta} s_{\iota}-\chi_{s}^{z} s_{\theta}^{2} s_{\iota}\right) \sin (\Psi)+\left(\chi_{s}^{x}\left(\frac{c_{\theta}^{2}}{2}+\frac{1}{2} c_{\theta}^{2} c_{\iota}\right)\right. \\
& \left.\left.+\chi_{s}^{z}\left(-\frac{1}{2} c_{\theta} s_{\theta}-\frac{1}{2} c_{\theta} c_{\iota} s_{\theta}\right)\right) \sin (\alpha+\Psi)\right] \\
& \hat{h}_{\times}^{(3 / 2, \mathrm{SO})}=\chi_{s}^{y}\left(2 c_{\iota}^{3} s_{\theta}-\nu c_{\iota}^{3} s_{\theta}\right)+\chi_{s}^{y} \cos (2 \alpha+2 \Psi)\left(\nu c_{\frac{\iota}{2}}^{4}\left(-\frac{5 s_{\theta}}{3}+\frac{2 c_{\iota} s_{\theta}}{3}\right)+c_{\frac{\iota}{2}}^{4}\left(-\frac{14 s_{\theta}}{3}+\frac{20 c_{\iota} s_{\theta}}{3}\right)\right) \\
& +\chi_{s}^{y} \cos (3 \alpha+2 \Psi)\left(-\frac{20}{3} c_{\theta} c_{\frac{\iota}{2}}^{5} s_{\frac{\iota}{2}}-\frac{2}{3} \nu c_{\theta} c_{\frac{\iota}{2}}^{5} s_{\frac{\iota}{2}}\right)+\chi_{s}^{y} \cos (\alpha+2 \Psi)\left(\nu c_{\frac{\iota}{2}}^{3}\left(\frac{7 c_{\theta}}{3}+\frac{c_{\theta} c_{\iota}}{3}\right) s_{\frac{\iota}{2}}\right.
\end{aligned}
$$




$$
\begin{aligned}
& \left.+c_{\frac{\iota}{2}}^{3}\left(-\frac{2 c_{\theta}}{3}+\frac{10 c_{\theta} c_{\iota}}{3}\right) s_{\frac{\iota}{2}}\right)+\chi_{s}^{y} \cos (\alpha-2 \Psi)\left(c_{\frac{\iota}{2}}\left(-\frac{2 c_{\theta}}{3}-\frac{10 c_{\theta} c_{\iota}}{3}\right) s_{\frac{\iota}{2}}^{3}+\nu c_{\frac{\iota}{2}}\left(\frac{7 c_{\theta}}{3}-\frac{c_{\theta} c_{\iota}}{3}\right) s_{\frac{\iota}{2}}^{3}\right) \\
& +\chi_{s}^{y} \cos (2 \alpha-2 \Psi)\left(\nu\left(\frac{5 s_{\theta}}{3}+\frac{2 c_{\iota} s_{\theta}}{3}\right) s_{\frac{\iota}{2}}^{4}+\left(\frac{14 s_{\theta}}{3}+\frac{20 c_{\iota} s_{\theta}}{3}\right) s_{\frac{\iota}{2}}^{4}\right) \\
& +\chi_{s}^{y} \cos (3 \alpha-2 \Psi)\left(-\frac{20}{3} c_{\theta} c_{\frac{\iota}{2}} s_{\frac{\iota}{2}}^{5}-\frac{2}{3} \nu c_{\theta} c_{\frac{\iota}{2}} s_{\frac{\iota}{2}}^{5}\right)+\chi_{s}^{y} \cos (2 \alpha)\left(2 c_{\iota} s_{\theta} s_{\iota}^{2}-\nu c_{\iota} s_{\theta} s_{\iota}^{2}\right) \\
& +\chi_{s}^{y} \cos (2 \Psi)\left(\frac{10}{3} c_{\iota} s_{\theta} s_{\iota}^{2}+\frac{1}{3} \nu c_{\iota} s_{\theta} s_{\iota}^{2}\right)+\chi_{s}^{y} \cos (3 \alpha)\left(-c_{\theta} s_{\iota}^{3}+\frac{1}{2} \nu c_{\theta} s_{\iota}^{3}\right)+\chi_{s}^{y} \cos (\alpha)\left(-\frac{5}{4} c_{\theta} s_{\iota}-\frac{1}{4} c_{\theta} s_{3 \iota}\right. \\
& \left.+\nu\left(\frac{5 c_{\theta} s_{\iota}}{8}+\frac{1}{8} c_{\theta} s_{3 \iota}\right)\right)+\left[\chi_{s}^{x}\left(\left(-\frac{3 c_{\theta}}{2}-\frac{1}{2} c_{\theta} c_{2 \iota}\right) s_{\iota}+\nu\left(\frac{3 c_{\theta}}{4}+\frac{1}{4} c_{\theta} c_{2 \iota}\right) s_{\iota}\right)\right. \\
& \left.+\chi_{s}^{z}\left(-2 c_{2 \iota} s_{\theta} s_{\iota}+\nu c_{2 \iota} s_{\theta} s_{\iota}\right)\right] \sin (\alpha)+\left(\chi_{s}^{z}\left(2 c_{\theta} c_{\iota} s_{\iota}^{2}-\nu c_{\theta} c_{\iota} s_{\iota}^{2}\right)+\chi_{s}^{x}\left(-2 c_{\iota} s_{\theta} s_{\iota}^{2}+\nu c_{\iota} s_{\theta} s_{\iota}^{2}\right)\right) \sin (2 \alpha) \\
& +\chi_{s}^{x}\left(c_{\theta} s_{\iota}^{3}-\frac{1}{2} \nu c_{\theta} s_{\iota}^{3}\right) \sin (3 \alpha)+\left[\chi_{s}^{x}\left(c_{\frac{\iota}{2}}\left(-\frac{2 c_{\theta}}{3}-\frac{10 c_{\theta} c_{\iota}}{3}\right) s_{\frac{\iota}{2}}^{3}+\nu c_{\frac{\iota}{2}}\left(-\frac{5 c_{\theta}}{3}+4 c_{3 \theta}-\frac{c_{\theta} c_{\iota}}{3}\right) s_{\frac{\iota}{2}}^{3}\right)\right. \\
& \left.+\chi_{s}^{z}\left(c_{\frac{\iota}{2}}\left(-4 s_{\theta}-\frac{40 c_{\iota} s_{\theta}}{3}\right) s_{\frac{\iota}{2}}^{3}+\nu c_{\frac{\iota}{2}}\left(-2 s_{\theta}-\frac{4 c_{\iota} s_{\theta}}{3}-4 s_{3 \theta}\right) s_{\frac{\iota}{2}}^{3}\right)\right] \sin (\alpha-2 \Psi) \\
& +\left[\chi_{s}^{z}\left(\nu\left(5 c_{\theta}+c_{3 \theta}+\frac{2 c_{\theta} c_{\iota}}{3}\right) s_{\frac{\iota}{2}}^{4}+\left(4 c_{\theta}+\frac{20 c_{\theta} c_{\iota}}{3}\right) s_{\frac{\iota}{2}}^{4}\right)+\chi_{s}^{x}\left(\left(-\frac{14 s_{\theta}}{3}-\frac{20 c_{\iota} s_{\theta}}{3}\right) s_{\frac{\iota}{2}}^{4}\right.\right. \\
& \left.\left.+\nu\left(\frac{10 s_{\theta}}{3}-\frac{2 c_{\iota} s_{\theta}}{3}+s_{3 \theta}\right) s_{\frac{\iota}{2}}^{4}\right)\right] \sin (2 \alpha-2 \Psi)+\chi_{s}^{x}\left(\frac{20}{3} c_{\theta} c_{\frac{\iota}{2}} s_{\frac{\iota}{2}}^{5}+\frac{2}{3} \nu c_{\theta} c_{\frac{\iota}{2}} s_{\frac{\iota}{2}}^{5}\right) \sin (3 \alpha-2 \Psi)+\left[-6 \nu \chi_{s}^{z} c_{\theta} s_{\theta}^{2} s_{\iota}^{2}\right. \\
& \left.+\chi_{s}^{x}\left(\frac{1}{3} s_{\theta} s_{\iota}^{2}+\nu\left(-\frac{7}{6}+3 c_{2 \theta}\right) s_{\theta} s_{\iota}^{2}\right)\right] \sin (2 \Psi)+\left[\chi _ { s } ^ { x } \left(\nu c_{\frac{\iota}{2}}^{3}\left(-\frac{5 c_{\theta}}{3}+4 c_{3 \theta}+\frac{c_{\theta} c_{\iota}}{3}\right) s_{\frac{\iota}{2}}\right.\right. \\
& \left.\left.+c_{\frac{\iota}{2}}^{3}\left(-\frac{2 c_{\theta}}{3}+\frac{10 c_{\theta} c_{\iota}}{3}\right) s_{\frac{\iota}{2}}\right)+\chi_{s}^{z}\left(c_{\frac{\iota}{2}}^{3}\left(-4 s_{\theta}+\frac{40 c_{\iota} s_{\theta}}{3}\right) s_{\frac{\iota}{2}}+\nu c_{\frac{\iota}{2}}^{3}\left(-2 s_{\theta}+\frac{4 c_{\iota} s_{\theta}}{3}-4 s_{3 \theta}\right) s_{\frac{\iota}{2}}\right)\right] \sin (\alpha+2 \Psi) \\
& +\left[\chi_{s}^{z}\left(\nu c_{\frac{L}{2}}^{4}\left(-5 c_{\theta}-c_{3 \theta}+\frac{2 c_{\theta} c_{\iota}}{3}\right)+c_{\frac{L}{2}}^{4}\left(-4 c_{\theta}+\frac{20 c_{\theta} c_{\iota}}{3}\right)\right)+\chi_{s}^{x}\left(c_{\frac{L}{2}}^{4}\left(\frac{14 s_{\theta}}{3}-\frac{20 c_{\iota} s_{\theta}}{3}\right)\right.\right. \\
& \left.\left.+\nu c_{\frac{\iota}{2}}^{4}\left(-\frac{10 s_{\theta}}{3}-\frac{2 c_{\iota} s_{\theta}}{3}-s_{3 \theta}\right)\right)\right] \sin (2 \alpha+2 \Psi)+\chi_{s}^{x}\left(\frac{20}{3} c_{\theta} c_{\frac{\iota}{2}}^{5} s_{\frac{\iota}{2}}+\frac{2}{3} \nu c_{\theta} c_{\frac{\iota}{2}}^{5} s_{\frac{\iota}{2}}\right) \sin (3 \alpha+2 \Psi) \\
& +\delta\left[2 \chi_{a}^{y} c_{\iota}^{3} s_{\theta}+\chi_{a}^{y} c_{\frac{\iota}{2}}^{4} \cos (2 \alpha+2 \Psi)\left(-\frac{14 s_{\theta}}{3}+\frac{20 c_{\iota} s_{\theta}}{3}\right)+\chi_{a}^{y} c_{\frac{\iota}{2}}^{3}\left(-\frac{2 c_{\theta}}{3}+\frac{10 c_{\theta} c_{\iota}}{3}\right) \cos (\alpha+2 \Psi) s_{\frac{\iota}{2}}\right. \\
& -\frac{20}{3} \chi_{a}^{y} c_{\theta} c_{\frac{\iota}{2}}^{5} \cos (3 \alpha+2 \Psi) s_{\frac{\iota}{2}}+\chi_{a}^{y} c_{\frac{\iota}{2}}\left(-\frac{2 c_{\theta}}{3}-\frac{10 c_{\theta} c_{\iota}}{3}\right) \cos (\alpha-2 \Psi) s_{\frac{\iota}{2}}^{3} \\
& +\chi_{a}^{y} \cos (2 \alpha-2 \Psi)\left(\frac{14 s_{\theta}}{3}+\frac{20 c_{\iota} s_{\theta}}{3}\right) s_{\frac{\iota}{2}}^{4}-\frac{20}{3} \chi_{a}^{y} c_{\theta} c_{\frac{\iota}{2}} \cos (3 \alpha-2 \Psi) s_{\frac{\iota}{2}}^{5}+2 \chi_{a}^{y} c_{\iota} \cos (2 \alpha) s_{\theta} s_{\iota}^{2} \\
& +\frac{10}{3} \chi_{a}^{y} c_{\iota} \cos (2 \Psi) s_{\theta} s_{\iota}^{2}-\chi_{a}^{y} c_{\theta} \cos (3 \alpha) s_{\iota}^{3}+\chi_{a}^{y} \cos (\alpha)\left(-\frac{5}{4} c_{\theta} s_{\iota}-\frac{1}{4} c_{\theta} s_{3 \iota}\right)+\left(\chi_{a}^{x}\left(-\frac{3 c_{\theta}}{2}-\frac{1}{2} c_{\theta} c_{2 \iota}\right) s_{\iota}\right. \\
& \left.-2 \chi_{a}^{z} c_{2 \iota} s_{\theta} s_{\iota}\right) \sin (\alpha)+\left(2 \chi_{a}^{z} c_{\theta} c_{\iota} s_{\iota}^{2}-2 \chi_{a}^{x} c_{\iota} s_{\theta} s_{\iota}^{2}\right) \sin (2 \alpha)+\chi_{a}^{x} c_{\theta} s_{\iota}^{3} \sin (3 \alpha)+\left(\chi_{a}^{x} c_{\frac{\iota}{2}}\left(-\frac{2 c_{\theta}}{3}-\frac{10 c_{\theta} c_{\iota}}{3}\right) s_{\frac{\iota}{2}}^{3}\right. \\
& \left.+\chi_{a}^{z} c_{\frac{\iota}{2}}\left(-4 s_{\theta}-\frac{40 c_{\iota} s_{\theta}}{3}\right) s_{\frac{\iota}{2}}^{3}\right) \sin (\alpha-2 \Psi)+\left(\chi_{a}^{z}\left(4 c_{\theta}+\frac{20 c_{\theta} c_{\iota}}{3}\right) s_{\frac{\frac{L}{2}}{2}}^{4}\right. \\
& \left.+\chi_{a}^{x}\left(-\frac{14 s_{\theta}}{3}-\frac{20 c_{\iota} s_{\theta}}{3}\right) s_{\frac{\iota}{2}}^{4}\right) \sin (2 \alpha-2 \Psi)+\frac{20}{3} \chi_{a}^{x} c_{\theta} c_{\frac{\iota}{2}} s_{\frac{\iota}{2}}^{5} \sin (3 \alpha-2 \Psi)+\frac{1}{3} \chi_{a}^{x} s_{\theta} s_{\iota}^{2} \sin (2 \Psi) \\
& +\left(\chi_{a}^{x} c_{\frac{\iota}{2}}^{3}\left(-\frac{2 c_{\theta}}{3}+\frac{10 c_{\theta} c_{\iota}}{3}\right) s_{\frac{\iota}{2}}+\chi_{a}^{z} c_{\frac{\iota}{2}}^{3}\left(-4 s_{\theta}+\frac{40 c_{\iota} s_{\theta}}{3}\right) s_{\frac{\iota}{2}}\right) \sin (\alpha+2 \Psi)+\left(\chi_{a}^{z} c_{\frac{\iota}{2}}^{4}\left(-4 c_{\theta}+\frac{20 c_{\theta} c_{\iota}}{3}\right)\right. \\
& \left.\left.+\chi_{a}^{x} c_{\frac{\iota}{2}}^{4}\left(\frac{14 s_{\theta}}{3}-\frac{20 c_{\iota} s_{\theta}}{3}\right)\right) \sin (2 \alpha+2 \Psi)+\frac{20}{3} \chi_{a}^{x} c_{\theta} c_{\frac{\iota}{2}}^{5} s_{\frac{\iota}{2}} \sin (3 \alpha+2 \Phi)\right] .
\end{aligned}
$$

where $s_{X}$ and $c_{X}$ are shorthand for $\sin X$ and $\cos X$, respectively, with $X=\theta, \iota, \ldots$ 


\section{Appendix B: Gravitational-wave modes for precessing binaries on nearly circular orbits through 1.5PN} order: generic inclination angles

In Sec. IV] we wrote the gravitational-wave modes expanded in the inclination angle $\iota$. Here we give the full expression of the $h_{22}, h_{33}$ and $h_{21}$ modes. They read

$$
\begin{aligned}
& h_{22}=-\frac{2 M \nu v^{2}}{D_{L}} e^{-2 i(\alpha+\Psi)} \frac{3}{2} \sqrt{\frac{\pi}{5}}\left\{\frac{1}{6} e^{-2 i \iota}\left(e^{4 i \Psi}\left(-1+e^{i \iota}\right)^{4}+\left(1+e^{i \iota}\right)^{4}\right)+\frac{1}{9} i e^{-i(2 \iota-\Psi)}\left[e^{2 i \Psi}\left(-1+e^{i \iota}\right)^{3}\left(1+e^{i \iota}\right)\right.\right. \\
& \left.-\left(-1+e^{i \iota}\right)\left(1+e^{i \iota}\right)^{3}\right] v \delta \\
& +v^{2}\left[\frac{1}{252} e^{-2 i \iota}\left(-107 e^{4 i \Psi}\left(-1+e^{i \iota}\right)^{4}-107\left(1+e^{i \iota}\right)^{4}\right)+\frac{55}{252} e^{-2 i \iota}\left(e^{4 i \Psi}\left(-1+e^{i \iota}\right)^{4}+\left(1+e^{i \iota}\right)^{4}\right) \eta\right. \\
& +\frac{1}{3} e^{i(\alpha-\iota+\Psi)}\left(e^{2 i \Psi}\left(-1+e^{i \iota}\right)^{2}-\left(1+e^{i \iota}\right)^{2}\right) \chi_{a}^{x}+\frac{1}{3} e^{i(\alpha-\iota+\Psi)}\left(e^{2 i \Psi}\left(-1+e^{i \iota}\right)^{2}-\left(1+e^{i \iota}\right)^{2}\right) \delta \chi_{s}^{x} \\
& \left.+i\left(\frac{1}{3} e^{i(\alpha-\iota+\Psi)}\left(-e^{2 i \Psi}\left(-1+e^{i \iota}\right)^{2}+\left(1+e^{i \iota}\right)^{2}\right) \chi_{a}^{y}+\frac{1}{3} e^{i(\alpha-\iota+\Psi)}\left(-e^{2 i \Psi}\left(-1+e^{i \iota}\right)^{2}+\left(1+e^{i \iota}\right)^{2}\right) \delta \chi_{s}^{y}\right)\right] \\
& +v^{3}\left[\frac{1}{3} e^{-2 i \iota}\left(e^{4 i \Psi}\left(-1+e^{i \iota}\right)^{4}+\left(1+e^{i \iota}\right)^{4}\right) \pi+\delta\left(\frac { 1 } { 3 6 } e ^ { - i ( \alpha + 3 \iota ) } \left(-5\left(-1+e^{i \iota}\right)\left(1+e^{i \iota}\right)^{5}\right.\right.\right. \\
& +e^{2 i \alpha}\left(1+e^{i \iota}\right)^{3}\left(-5+7 e^{i \iota}-7 e^{2 i \iota}+5 e^{3 i \iota}\right)+e^{4 i \Psi}\left(-1+e^{i \iota}\right)^{3}\left(1+e^{i \iota}\right)\left(-5+5 e^{2 i \alpha}+5 e^{2 i \iota}\left(-1+e^{2 i \alpha}\right)\right. \\
& \left.\left.+2 e^{i \iota}\left(5+e^{2 i \alpha}\right)\right)+6 e^{2 i \Psi}\left(\left(-1+e^{2 i \iota}\right)^{3}-e^{2 i \alpha}\left(-1-5 e^{2 i \iota}+5 e^{4 i \iota}+e^{6 i \iota}\right)\right)\right) \chi_{a}^{y} \\
& +\frac{1}{18} e^{-3 i \iota}\left(6 e^{2 i \Psi}\left(-1+e^{2 i \iota}\right)^{2}\left(1+e^{2 i \iota}\right)-\left(1+e^{i \iota}\right)^{4}\left(5-6 e^{i \iota}+5 e^{2 i \iota}\right)\right. \\
& \left.\left.-e^{4 i \Psi}\left(-1+e^{i \iota}\right)^{4}\left(5+6 e^{i \iota}+5 e^{2 i \iota}\right)\right) \chi_{a}^{z}\right) \\
& +\frac{1}{36} e^{-i(\alpha+3 \iota)}\left(-5\left(-1+e^{i \iota}\right)\left(1+e^{i \iota}\right)^{5}+e^{2 i \alpha}\left(1+e^{i \iota}\right)^{3}\left(-5+7 e^{i \iota}-7 e^{2 i \iota}+5 e^{3 i \iota}\right)\right. \\
& +e^{4 i \Psi}\left(-1+e^{i \iota}\right)^{3}\left(1+e^{i \iota}\right)\left(-5+5 e^{2 i \alpha}+5 e^{2 i \iota}\left(-1+e^{2 i \alpha}\right)+2 e^{i \iota}\left(5+e^{2 i \alpha}\right)\right)+6 e^{2 i \Psi}\left(\left(-1+e^{2 i \iota}\right)^{3}\right. \\
& \left.\left.-e^{2 i \alpha}\left(-1-5 e^{2 i \iota}+5 e^{4 i \iota}+e^{6 i \iota}\right)\right)\right) \chi_{s}^{y}+\frac{1}{18} e^{-3 i \iota}\left(6 e^{2 i \Psi}\left(-1+e^{2 i \iota}\right)^{2}\left(1+e^{2 i \iota}\right)-\left(1+e^{i \iota}\right)^{4}\left(5-6 e^{i \iota}+5 e^{2 i \iota}\right)\right. \\
& \left.-e^{4 i \Psi}\left(-1+e^{i \iota}\right)^{4}\left(5+6 e^{i \iota}+5 e^{2 i \iota}\right)\right) \chi_{s}^{z}+i\left(\delta \left(\frac { 1 } { 2 5 2 } e ^ { - i ( 2 \iota - \Psi ) } \left(-17 e^{2 i \Psi}\left(-1+e^{i \iota}\right)^{3}\left(1+e^{i \iota}\right)\right.\right.\right. \\
& \left.+17\left(-1+e^{i \iota}\right)\left(1+e^{i \iota}\right)^{3}\right)+\frac{1}{63} e^{-i(2 \iota-\Psi)}\left(5 e^{2 i \Psi}\left(-1+e^{i \iota}\right)^{3}\left(1+e^{i \iota}\right)-5\left(-1+e^{i \iota}\right)\left(1+e^{i \iota}\right)^{3}\right) \eta \\
& +\frac{1}{36} e^{-i(\alpha+3 \iota)}\left(5\left(-1+e^{i \iota}\right)\left(1+e^{i \iota}\right)^{5}+e^{2 i \alpha}\left(1+e^{i \iota}\right)^{3}\left(-5+7 e^{i \iota}-7 e^{2 i \iota}+5 e^{3 i \iota}\right)\right. \\
& +e^{4 i \Psi}\left(-1+e^{i \iota}\right)^{3}\left(1+e^{i \iota}\right)\left(5\left(-1+e^{i \iota}\right)^{2}+e^{2 i \alpha}\left(5+2 e^{i \iota}+5 e^{2 i \iota}\right)\right)+6 e^{2 i \Psi}\left(-\left(-1+e^{2 i \iota}\right)^{3}\right. \\
& \left.\left.\left.-e^{2 i \alpha}\left(-1-5 e^{2 i \iota}+5 e^{4 i \iota}+e^{6 i \iota}\right)\right)\right) \chi_{a}^{x}\right)+\frac{1}{36} e^{-i(\alpha+3 \iota)}\left(5\left(-1+e^{i \iota}\right)\left(1+e^{i \iota}\right)^{5}\right. \\
& +e^{2 i \alpha}\left(1+e^{i \iota}\right)^{3}\left(-5+7 e^{i \iota}-7 e^{2 i \iota}+5 e^{3 i \iota}\right)+e^{4 i \Psi}\left(-1+e^{i \iota}\right)^{3}\left(1+e^{i \iota}\right) \\
& \times\left(5\left(-1+e^{i \iota}\right)^{2}+e^{2 i \alpha}\left(5+2 e^{i \iota}+5 e^{2 i \iota}\right)\right) \\
& \left.+6 e^{2 i \Psi}\left(-\left(-1+e^{2 i \iota}\right)^{3}-e^{2 i \alpha}\left(-1-5 e^{2 i \iota}+5 e^{4 i \iota}+e^{6 i \iota}\right)\right)\right) \chi_{s}^{x}+\frac{1}{72} e^{-i(\alpha+3 \iota)}\left(( - 1 + e ^ { i \iota } ) ( 1 + e ^ { i \iota } ) ^ { 3 } \left(1+e^{2 i \alpha}\right.\right. \\
& \left.+e^{2 i \iota}\left(1+e^{2 i \alpha}\right)-2 e^{i \iota}\left(-1+9 e^{2 i \alpha}\right)\right)+e^{4 i \Psi}\left(-1+e^{i \iota}\right)^{3}\left(1+e^{i \iota}\right) \\
& \times\left(1+e^{2 i \alpha}+e^{2 i \iota}\left(1+e^{2 i \alpha}\right)+2 e^{i \iota}\left(-1+9 e^{2 i \alpha}\right)\right) \\
& \left.\left.+6 e^{2 i \Psi}\left(\left(-1+e^{2 i \iota}\right)^{3}+e^{2 i \alpha}\left(-1-5 e^{2 i \iota}+5 e^{4 i \iota}+e^{6 i \iota}\right)\right)\right) \eta \chi_{s}^{x}\right)+\eta\left(\frac { 1 } { 7 2 } e ^ { - i ( \alpha + 3 \iota ) } \left(-\left(-1+e^{i \iota}\right)\left(1+e^{i \iota}\right)^{5}\right.\right. \\
& +e^{2 i \alpha}\left(1+e^{i \iota}\right)^{3}\left(-1+19 e^{i \iota}-19 e^{2 i \iota}+e^{3 i \iota}\right)+e^{4 i \Psi}\left(-1+e^{i \iota}\right)^{3}\left(1+e^{i \iota}\right)\left(-1+e^{2 i \alpha}+e^{2 i \iota}\left(-1+e^{2 i \alpha}\right)\right.
\end{aligned}
$$




$$
\begin{aligned}
& \left.\left.+2 e^{i \iota}\left(1+9 e^{2 i \alpha}\right)\right)+6 e^{2 i \Psi}\left(-\left(-1+e^{2 i \iota}\right)^{3}+e^{2 i \alpha}\left(-1-5 e^{2 i \iota}+5 e^{4 i \iota}+e^{6 i \iota}\right)\right)\right) \chi_{s}^{y} \\
& +\frac{1}{36} e^{-3 i \iota}\left(-6 e^{2 i \Psi}\left(-1+e^{2 i \iota}\right)^{2}\left(1+e^{2 i \iota}\right)-\left(1+e^{i \iota}\right)^{4}\left(1-10 e^{i \iota}+e^{2 i \iota}\right)\right. \\
& \left.\left.\left.\left.-e^{4 i \Psi}\left(-1+e^{i \iota}\right)^{4}\left(1+10 e^{i \iota}+e^{2 i \iota}\right)\right) \chi_{s}^{z}\right)\right]+\mathcal{O}\left(v^{4}\right)\right\}
\end{aligned}
$$

$$
\begin{aligned}
& h_{21}=-\frac{2 M \nu v^{2}}{D_{L}} e^{-i(\alpha+\Psi)} i \sqrt{\frac{\pi}{5}}\left\{\frac{1}{2} e^{-i(2 \iota+\Psi)}\left(-e^{4 i \Psi}\left(-1+e^{i \iota}\right)^{3}\left(1+e^{i \iota}\right)-\left(-1+e^{i \iota}\right)\left(1+e^{i \iota}\right)^{3}\right)\right. \\
& +\frac{1}{3} i e^{-2 i \iota}\left[1+e^{i \iota}+e^{3 i \iota}+e^{4 i \iota}-e^{2 i \Psi}\left(-1+e^{i \iota}\right)^{2}\left(1+e^{i \iota}+e^{2 i \iota}\right)\right] v \delta \\
& +v^{2}\left[\frac{107}{84} e^{-i(2 \iota+\Psi)}\left(-1+e^{i \iota}\right)\left(1+e^{i \iota}\right)\left(e^{4 i \Psi}\left(-1+e^{i \iota}\right)^{2}+\left(1+e^{i \iota}\right)^{2}\right)+\frac{1}{84} e^{-i(2 \iota+\Psi)}\left(-55 e^{4 i \Psi}\left(-1+e^{i \iota}\right)^{3}\right.\right. \\
& \left.\times\left(1+e^{i \iota}\right)-55\left(-1+e^{i \iota}\right)\left(1+e^{i \iota}\right)^{3}\right) \eta-\frac{1}{2} e^{i(\alpha-\iota)}\left(-1+e^{2 i \iota}\right)\left(-1+e^{2 i \Psi}\right) \chi_{a}^{x} \\
& -\frac{1}{2} e^{i(\alpha-\iota)}\left(-1+e^{2 i \iota}\right)\left(-1+e^{2 i \Psi}\right) \delta \chi_{s}^{x}+i\left(\frac{1}{2} e^{i(\alpha-\iota)}\left(-1+e^{2 i \iota}\right)\left(-1+e^{2 i \Psi}\right) \chi_{a}^{y}\right. \\
& +\frac{1}{2} e^{-i \iota}\left(e^{2 i \Psi}\left(-1+e^{i \iota}\right)^{2}-\left(1+e^{i \iota}\right)^{2}\right) \chi_{a}^{z}+\delta\left(\frac{1}{2} e^{i(\alpha-\iota)}\left(-1+e^{2 i \iota}\right)\left(-1+e^{2 i \Psi}\right) \chi_{s}^{y}\right. \\
& \left.\left.\left.+\frac{1}{2} e^{-i \iota}\left(e^{2 i \Psi}\left(-1+e^{i \iota}\right)^{2}-\left(1+e^{i \iota}\right)^{2}\right) \chi_{s}^{z}\right)\right)\right] \\
& +v^{3}\left[e^{-i(2 \iota+\Psi)}\left(-e^{4 i \Psi}\left(-1+e^{i \iota}\right)^{3}\left(1+e^{i \iota}\right) \pi-\left(-1+e^{i \iota}\right)\left(1+e^{i \iota}\right)^{3} \pi\right)\right. \\
& +\delta\left(\frac { 1 } { 1 2 } e ^ { - i ( \alpha + 3 \iota + \Psi ) } \left(\left(1+e^{i \iota}\right)^{4}\left(5-7 e^{i \iota}+5 e^{2 i \iota}\right)-e^{2 i \alpha}\left(-1+e^{2 i \iota}\right)^{2}\left(5+e^{i \iota}+5 e^{2 i \iota}\right)\right.\right. \\
& +6 e^{2 i \Psi}\left(1+e^{2 i \iota}\right)\left(-\left(-1+e^{2 i \iota}\right)^{2}\right. \\
& \left.\left.+e^{2 i \alpha}\left(1+e^{2 i \iota}\right)^{2}\right)+e^{4 i \Psi}\left(-e^{2 i \alpha}\left(-1+e^{2 i \iota}\right)^{2}\left(5-e^{i \iota}+5 e^{2 i \iota}\right)+\left(-1+e^{i \iota}\right)^{4}\left(5+7 e^{i \iota}+5 e^{2 i \iota}\right)\right)\right) \chi_{a}^{y} \\
& +\frac{1}{6} e^{-i(3 \iota+\Psi)}\left(\left(1+e^{i \iota}\right)^{3}\left(-5+8 e^{i \iota}-8 e^{2 i \iota}+5 e^{3 i \iota}\right)+e^{4 i \Psi}\left(-1+e^{i \iota}\right)^{3}\left(5+8 e^{i \iota}+8 e^{2 i \iota}+5 e^{3 i \iota}\right)\right. \\
& \left.\left.-6 e^{2 i \Psi}\left(-1+e^{2 i \iota}-e^{4 i \iota}+e^{6 i \iota}\right)\right) \chi_{a}^{z}\right)+\frac{1}{12} e^{-i(\alpha+3 \iota+\Psi)}\left(\left(1+e^{i \iota}\right)^{4}\left(5-7 e^{i \iota}+5 e^{2 i \iota}\right)\right. \\
& -e^{2 i \alpha}\left(-1+e^{2 i \iota}\right)^{2}\left(5+e^{i \iota}+5 e^{2 i \iota}\right)+6 e^{2 i \Psi}\left(1+e^{2 i \iota}\right)\left(-\left(-1+e^{2 i \iota}\right)^{2}+e^{2 i \alpha}\left(1+e^{2 i \iota}\right)^{2}\right) \\
& \left.+e^{4 i \Psi}\left(-e^{2 i \alpha}\left(-1+e^{2 i \iota}\right)^{2}\left(5-e^{i \iota}+5 e^{2 i \iota}\right)+\left(-1+e^{i \iota}\right)^{4}\left(5+7 e^{i \iota}+5 e^{2 i \iota}\right)\right)\right) \chi_{s}^{y} \\
& +\frac{1}{6} e^{-i(3 \iota+\Psi)}\left(\left(1+e^{i \iota}\right)^{3}\left(-5+8 e^{i \iota}-8 e^{2 i \iota}+5 e^{3 i \iota}\right)+e^{4 i \Psi}\left(-1+e^{i \iota}\right)^{3}\left(5+8 e^{i \iota}+8 e^{2 i \iota}+5 e^{3 i \iota}\right)\right. \\
& \left.-6 e^{2 i \Psi}\left(-1+e^{2 i \iota}-e^{4 i \iota}+e^{6 i \iota}\right)\right) \chi_{s}^{z}+i\left(\delta \left(\frac { 1 } { 8 4 } e ^ { - 2 i \iota } \left(-17+17 e^{2 i \Psi}\left(-1+e^{i \iota}\right)^{2}\left(1+e^{i \iota}+e^{2 i \iota}\right)\right.\right.\right. \\
& \left.-17 e^{i \iota}\left(1+e^{2 i \iota}+e^{3 i \iota}\right)\right)+\frac{5}{21} e^{-2 i \iota}\left(1+e^{i \iota}+e^{3 i \iota}+e^{4 i \iota}-e^{2 i \Psi}\left(-1+e^{i \iota}\right)^{2}\left(1+e^{i \iota}+e^{2 i \iota}\right)\right) \eta \\
& +\frac{1}{12} e^{-i(\alpha+3 \iota+\Psi)}\left(-\left(1+e^{i \iota}\right)^{4}\left(5-7 e^{i \iota}+5 e^{2 i \iota}\right)-e^{2 i \alpha}\left(-1+e^{2 i \iota}\right)^{2}\left(5+e^{i \iota}+5 e^{2 i \iota}\right)\right. \\
& +6 e^{2 i \Psi}\left(1+e^{2 i \iota}\right)\left(\left(-1+e^{2 i \iota}\right)^{2}+e^{2 i \alpha}\left(1+e^{2 i \iota}\right)^{2}\right)+e^{4 i \Psi}\left(-e^{2 i \alpha}\left(-1+e^{2 i \iota}\right)^{2}\left(5-e^{i \iota}+5 e^{2 i \iota}\right)\right. \\
& \left.\left.\left.-\left(-1+e^{i \iota}\right)^{4}\left(5+7 e^{i \iota}+5 e^{2 i \iota}\right)\right)\right) \chi_{a}^{x}\right)+\frac{1}{12} e^{-i(\alpha+3 \iota+\Psi)}\left(-\left(1+e^{i \iota}\right)^{4}\left(5-7 e^{i \iota}+5 e^{2 i \iota}\right)\right. \\
& -e^{2 i \alpha}\left(-1+e^{2 i \iota}\right)^{2}\left(5+e^{i \iota}+5 e^{2 i \iota}\right)+6 e^{2 i \Psi}\left(1+e^{2 i \iota}\right)\left(\left(-1+e^{2 i \iota}\right)^{2}+e^{2 i \alpha}\left(1+e^{2 i \iota}\right)^{2}\right) \\
& \left.+e^{4 i \Psi}\left(-e^{2 i \alpha}\left(-1+e^{2 i \iota}\right)^{2}\left(5-e^{i \iota}+5 e^{2 i \iota}\right)-\left(-1+e^{i \iota}\right)^{4}\left(5+7 e^{i \iota}+5 e^{2 i \iota}\right)\right)\right) \chi_{s}^{x}
\end{aligned}
$$




$$
\begin{aligned}
& +\frac{1}{24} e^{-i(\alpha+3 \iota+\Psi)}\left(-e^{2 i \alpha}\left(-1+e^{2 i \iota}\right)^{2}\left(1-13 e^{i \iota}+e^{2 i \iota}\right)-\left(1+e^{i \iota}\right)^{4}\left(1+3 e^{i \iota}+e^{2 i \iota}\right)\right. \\
& +6 e^{2 i \Psi}\left(1+e^{2 i \iota}\right)\left(-\left(-1+e^{2 i \iota}\right)^{2}-e^{2 i \alpha}\left(1+e^{2 i \iota}\right)^{2}\right)+e^{4 i \Psi}\left(-\left(-1+e^{i \iota}\right)^{4}\left(1-3 e^{i \iota}+e^{2 i \iota}\right)\right. \\
& \left.\left.\left.-e^{2 i \alpha}\left(-1+e^{2 i \iota}\right)^{2}\left(1+13 e^{i \iota}+e^{2 i \iota}\right)\right)\right) \eta \chi_{s}^{x}\right)+\eta\left(\frac { 1 } { 2 4 } e ^ { - i ( \alpha + 3 \iota + \Psi ) } \left(-e^{2 i \alpha}\left(-1+e^{2 i \iota}\right)^{2}\left(1-13 e^{i \iota}+e^{2 i \iota}\right)\right.\right. \\
& +\left(1+e^{i \iota}\right)^{4}\left(1+3 e^{i \iota}+e^{2 i \iota}\right)+6 e^{2 i \Psi}\left(1+e^{2 i \iota}\right)\left(\left(-1+e^{2 i \iota}\right)^{2}-e^{2 i \alpha}\left(1+e^{2 i \iota}\right)^{2}\right) \\
& \left.+e^{4 i \Psi}\left(\left(-1+e^{i \iota}\right)^{4}\left(1-3 e^{i \iota}+e^{2 i \iota}\right)-e^{2 i \alpha}\left(-1+e^{2 i \iota}\right)^{2}\left(1+13 e^{i \iota}+e^{2 i \iota}\right)\right)\right) \chi_{s}^{y} \\
& +\frac{1}{12} e^{-i(3 \iota+\Psi)}\left(\left(1+e^{i \iota}\right)^{3}\left(-1+6 e^{i \iota}-6 e^{2 i \iota}+e^{3 i \iota}\right)+e^{4 i \Psi}\left(-1+e^{i \iota}\right)^{3}\left(1+6 e^{i \iota}+6 e^{2 i \iota}+e^{3 i \iota}\right)\right. \\
& \left.\left.\left.\left.+6 e^{2 i \Psi}\left(-1+e^{2 i \iota}-e^{4 i \iota}+e^{6 i \iota}\right)\right) \chi_{s}^{z}\right)\right]+\mathcal{O}\left(v^{4}\right)\right\}
\end{aligned}
$$

$$
\begin{aligned}
h_{33}= & \frac{2 M \nu v^{2}}{D_{L}} e^{-3 i(\alpha+\Psi)} \frac{1}{64} \sqrt{\frac{\pi}{42}}\left\{e ^ { - 3 i \iota } \left(9 e^{6 i \Psi}\left(-1+e^{i \iota}\right)^{6}-e^{4 i \Psi}\left(-1+e^{i \iota}\right)^{4}\left(1+e^{i \iota}\right)^{2}\right.\right. \\
& \left.-e^{2 i \Psi}\left(-1+e^{i \iota}\right)^{2}\left(1+e^{i \iota}\right)^{4}+9\left(1+e^{i \iota}\right)^{6}\right) v \delta \\
& +i v^{2}\left[e^{-i(3 \iota-\Psi)}\left(8 e^{4 i \Psi}\left(-1+e^{i \iota}\right)^{5}\left(1+e^{i \iota}\right)-8\left(-1+e^{i \iota}\right)\left(1+e^{i \iota}\right)^{5}\right)\right. \\
& +e^{-i(3 \iota-\Psi)}\left(-24 e^{4 i \Psi}\left(-1+e^{i \iota}\right)^{5}\left(1+e^{i \iota}\right)\right. \\
& \left.\left.+24\left(-1+e^{i \iota}\right)\left(1+e^{i \iota}\right)^{5}\right) \eta\right]+v^{3}\left[\delta \left(\frac { 1 } { 3 } e ^ { - 3 i \iota } \left(-108 e^{6 i \Psi}\left(-1+e^{i \iota}\right)^{6}+8 e^{4 i \Psi}\left(-1+e^{i \iota}\right)^{4}\left(1+e^{i \iota}\right)^{2}\right.\right.\right. \\
& \left.+8 e^{2 i \Psi}\left(-1+e^{i \iota}\right)^{2}\left(1+e^{i \iota}\right)^{4}-108\left(1+e^{i \iota}\right)^{6}\right)+\frac{1}{3} e^{-3 i \iota}\left(54 e^{6 i \Psi}\left(-1+e^{i \iota}\right)^{6}+2 e^{4 i \Psi}\left(-1+e^{i \iota}\right)^{4}\left(1+e^{i \iota}\right)^{2}\right. \\
& \left.\left.+2 e^{2 i \Psi}\left(-1+e^{i \iota}\right)^{2}\left(1+e^{i \iota}\right)^{4}+54\left(1+e^{i \iota}\right)^{6}\right) \eta\right)+64 e^{i(\alpha-2 \iota+\Psi)}\left(-e^{4 i \Psi}\left(-1+e^{i \iota}\right)^{4}+\left(1+e^{i \iota}\right)^{4}\right)^{x} \chi_{s}^{x} \\
& \left.\left.+64 i e^{i(\alpha-2 \iota+\Psi)}\left(e^{4 i \Psi}\left(-1+e^{i \iota}\right)^{4}-\left(1+e^{i \iota}\right)^{4}\right) \eta \chi_{s}^{y}\right]+\mathcal{O}\left(v^{4}\right)\right\}
\end{aligned}
$$

\section{Appendix C: Center-of-mass energy and gravitational-wave energy flux}

For nearly circular orbits, the center-of-mass energy is known through 2PN order, when spins are present and 3PN order when spins are neglected. The coefficients entering Eq. (6.18) are [29, 34, 35, 75, 82 86]

$$
\begin{aligned}
E_{\text {Newt }} & =-\frac{M}{2} \nu \\
E_{2} & =-\frac{3}{4}-\frac{1}{12} \nu \\
E_{3} & =\left[\left(\frac{8}{3}-\frac{4}{3} \nu\right) \boldsymbol{\chi}_{s} \cdot \hat{\mathbf{L}}_{\mathrm{N}}+\frac{8}{3} \delta \boldsymbol{\chi}_{a} \cdot \hat{\mathbf{L}}_{\mathrm{N}}\right] \\
E_{4} & =-\frac{27}{8}+\frac{19}{8} \nu-\frac{1}{24} \nu^{2}+\nu\left\{\left(\boldsymbol{\chi}_{s}^{2}-\boldsymbol{\chi}_{a}^{2}\right)-3\left[\left(\boldsymbol{\chi}_{s} \cdot \hat{\mathbf{L}}_{\mathrm{N}}\right)^{2}-\left(\boldsymbol{\chi}_{a} \cdot \hat{\mathbf{L}}_{\mathrm{N}}\right)^{2}\right]\right\} \\
& +\left(\frac{1}{2}-\nu\right)\left\{\chi_{s}^{2}+\boldsymbol{\chi}_{a}^{2}-3\left[\left(\boldsymbol{\chi}_{s} \cdot \hat{\mathbf{L}}_{\mathrm{N}}\right)^{2}+\left(\boldsymbol{\chi}_{a} \cdot \hat{\mathbf{L}}_{\mathrm{N}}\right)^{2}\right]\right\} \\
+ & \delta\left\{\boldsymbol{\chi}_{s} \cdot \boldsymbol{\chi}_{a}-3\left[\left(\boldsymbol{\chi}_{s} \cdot \hat{\mathbf{L}}_{\mathrm{N}}\right)\left(\boldsymbol{\chi}_{a} \cdot \hat{\mathbf{L}}_{\mathrm{N}}\right)\right]\right\} \\
E_{5} & =\left[\left(8-\frac{121}{9} \nu+\frac{2}{9} \nu^{2}\right) \boldsymbol{\chi}_{s} \cdot \hat{\mathbf{L}}_{\mathrm{N}}+\left(8-\frac{31}{9} \nu\right) \delta \boldsymbol{\chi}_{a} \cdot \hat{\mathbf{L}}_{\mathrm{N}}\right] \\
E_{6} & =-\frac{675}{64}+\left(\frac{34445}{576}-\frac{205}{96} \pi^{2}\right) \nu-\frac{155}{96} \nu^{2}-\frac{35}{5184} \nu^{3} .
\end{aligned}
$$


The GW energy flux is known through 2.5PN order for spin effects [29, 30, 32, 33], and 3.5PN order when spin effects are absent [23]. The coefficients in Eq. (6.19) read

$$
\begin{aligned}
F_{\text {Newt }}= & \frac{32}{5} \nu^{2} \\
F_{2}= & -\frac{1247}{336}-\frac{35}{12} \nu \\
F_{3}= & 4 \pi-\left[\left(\frac{11}{4}-3 \nu\right) \chi_{s} \cdot \hat{\mathbf{L}}_{\mathrm{N}}+\frac{11}{4} \delta \boldsymbol{\chi}_{a} \cdot \hat{\mathbf{L}}_{\mathrm{N}}\right] \\
F_{4}= & -\frac{44711}{9072}+\frac{9271}{504} \nu+\frac{65}{18} \nu^{2}+\left(\frac{287}{96}+\frac{\nu}{24}\right)\left(\chi_{s} \cdot \hat{\mathbf{L}}_{\mathrm{N}}\right)^{2}-\left(\frac{89}{96}+\frac{7 \nu}{24}\right) \chi_{s}^{2} \\
& +\left(\frac{287}{96}-12 \nu\right)\left(\chi_{a} \cdot \hat{\mathbf{L}}_{\mathrm{N}}\right)^{2}+\left(-\frac{89}{96}+4 \nu\right) \chi_{a}^{2}+\frac{287}{48} \delta\left(\chi_{s} \cdot \hat{\mathbf{L}}_{\mathrm{N}}\right)\left(\chi_{a} \cdot \hat{\mathbf{L}}_{\mathrm{N}}\right) \\
& -\frac{89}{48} \delta\left(\chi_{s} \cdot \chi_{a}\right) \\
F_{5}= & \left(-\frac{8191}{672}-\frac{583}{24} \nu\right) \pi+\left[\left(-\frac{59}{16}+\frac{227}{9} \nu-\frac{157}{9} \nu^{2}\right) \chi_{s} \cdot \hat{\mathbf{L}}_{\mathrm{N}}+\left(-\frac{59}{16}+\frac{701}{36} \nu\right) \delta \chi_{a} \cdot \hat{\mathbf{L}}_{\mathrm{N}}\right] \\
F_{6}= & \frac{6643739519}{69854400}+\frac{16}{3} \pi^{2}-\frac{1712}{105} \gamma_{E}-\frac{856}{105} \log \left(16 v^{2}\right)+\left(-\frac{134543}{7776}+\frac{41}{48} \pi^{2}\right) \nu-\frac{94403}{3024} \nu^{2}-\frac{775}{324} \nu^{3}, \\
F_{7}= & \left(-\frac{16285}{504}+\frac{214745}{1728} \nu+\frac{193385}{3024} \nu^{2}\right) \pi .
\end{aligned}
$$

\section{Appendix D: Frequency-domain amplitude corrections}

We give here the complex coefficients $\mathcal{C}_{k}^{(n)}$ appearing in the frequency domain non-precessing waveform (6.13). The lower index in $\mathcal{C}_{k}^{(n)}$ denotes the harmonic of the orbital phase, and the upper index denotes the (half) PN order. Since the different harmonics end at different GW frequencies, the $k^{\text {th }}$ harmonic ends at $k$ times the orbital frequency cutoff. Thus, we introduce step functions $\Theta\left(k F_{\text {cut }}-f\right)$ to ensure each harmonic ends at its proper frequency. We derive

$$
\begin{gathered}
\mathcal{C}_{2}^{(0)}=\frac{1}{\sqrt{2}}\left[-\left(1+c_{\theta}^{2}\right) F_{+}-2 i c_{\theta} F_{\times}\right] \Theta\left(2 F_{\text {cut }}-f\right) \\
\mathcal{C}_{1}^{(1)}=s_{\theta} \delta\left[-\left(\frac{5}{8}+\frac{c_{\theta}^{2}}{8}\right) F_{+}-\frac{3}{4} i c_{\theta} F_{\times}\right] \Theta\left(F_{\text {cut }}-f\right) \\
\mathcal{C}_{3}^{(1)}=\frac{1}{\sqrt{3}}\left[\frac{9}{8} s_{\theta} \delta\left(\left(1+c_{\theta}^{2}\right) F_{+}+i 2 c_{\theta} F_{\times}\right)\right] \Theta\left(3 F_{\text {cut }}-f\right) \\
\mathcal{C}_{1}^{(2)}=s_{\theta}\left(\delta \chi_{s} \cdot \hat{\mathbf{L}}_{\mathrm{N}}+\chi_{a} \cdot \hat{\mathbf{L}}_{\mathrm{N}}\right)\left(F_{+}+i c_{\theta} F_{\times}\right) \Theta\left(F_{\text {cut }}-f\right) \\
\mathcal{C}_{2}^{(2)}=\frac{1}{\sqrt{2}}\left[\left(\frac{1385}{672}-\frac{109 \nu}{24}+\left(\frac{265}{672}+\frac{11 \nu}{24}\right) c_{\theta}^{2}+\left(-\frac{1}{3}+\nu\right) c_{\theta}^{4}\right) F_{+}\right. \\
\left.+i\left(\left(\frac{387}{112}-\frac{85}{12} \nu\right) c_{\theta}+\left(-\frac{4}{3}+4 \nu\right) c_{\theta}^{3}\right) F_{\times}\right] \Theta\left(2 F_{\text {cut }}-f\right) \\
\mathcal{C}_{4}^{(2)}=\frac{1}{2} s_{\theta}^{2}\left[\frac{4}{3}(3 \nu-1)\left(\left(1+c_{\theta}^{2}\right) F_{+}+i 2 c_{\theta} F_{\times}\right)\right] \Theta\left(4 F_{\text {cut }}-f\right) \\
\mathcal{C}_{1}^{(3)}=s_{\theta} \delta\left[\left(-\frac{2119}{5376}-\frac{263 \nu}{192}+\left(\frac{937}{5376}-\frac{3 \nu}{64}\right) c_{\theta}^{2}+\left(-\frac{1}{192}+\frac{\nu}{96}\right) c_{\theta}^{4}\right) F_{+}\right.
\end{gathered}
$$




$$
\begin{aligned}
& \left.+i\left(-\left(\frac{155}{896}+\frac{145 \nu}{96}\right) c_{\theta}+\frac{5}{96}(2 \nu-1) c_{\theta}^{3}\right) F_{\times}\right] \Theta\left(F_{\text {cut }}-f\right) \\
& \mathcal{C}_{2}^{(3)}=\frac{1}{\sqrt{2}}\left[\left(-\frac{27}{8}\left(1+c_{\theta}^{2}\right) \delta\left(\chi_{a} \cdot \hat{\mathbf{L}}_{\mathrm{N}}\right)+\left(-\frac{27}{8}\left(1+c_{\theta}^{2}\right)+\frac{1}{2}\left(9-7 c_{\theta}^{2}\right) \nu\right)\left(\chi_{s} \cdot \hat{\mathbf{L}}_{\mathrm{N}}\right)\right) F_{+}\right. \\
& \left.+i c_{\theta}\left(-\frac{27}{4} \delta\left(\chi_{a} \cdot \hat{\mathbf{L}}_{\mathrm{N}}\right)+\left(-\frac{27}{4}+\left(5-4 c_{\theta}^{2}\right) \nu\right)\left(\chi_{s} \cdot \hat{\mathbf{L}}_{\mathrm{N}}\right)\right) F_{\times}\right] \Theta\left(2 F_{\text {cut }}-f\right), \\
& \mathcal{C}_{3}^{(3)}=\frac{1}{\sqrt{3}} s_{\theta} \delta\left[\left(-\frac{6969}{1792}+\frac{81 \nu}{16}+\left(-\frac{2811}{1792}+\frac{27 \nu}{64}\right) c_{\theta}^{2}+\frac{81}{128}(1-2 \nu) c_{\theta}^{4}\right) F_{+}\right. \\
& \left.+i\left(\left(-\frac{6213}{896}+\frac{135 \nu}{16}\right) c_{\theta}+\frac{135}{64}(1-2 \nu) c_{\theta}^{3}\right) F_{\times}\right] \Theta\left(3 F_{\text {cut }}-f\right), \\
& \mathcal{C}_{5}^{(3)}=\frac{1}{\sqrt{5}} s_{\theta}^{3} \delta(1-2 \nu)\left[\frac{625}{384}\left(\left(1+c_{\theta}^{2}\right) F_{+}+i 2 c_{\theta} F_{\times}\right)\right] \Theta\left(5 F_{\text {cut }}-f\right) \\
& \mathcal{C}_{1}^{(4)}=s_{\theta} \delta\left[\left(\frac{5 \pi}{8}+i\left(\frac{11}{40}+\frac{5 \ln 2}{4}\right)+\left(\frac{\pi}{8}+i\left(\frac{7}{40}+\frac{\ln 2}{4}\right)\right) c_{\theta}^{2}\right) F_{+}\right. \\
& \left.+\left(-\left(\frac{9}{20}+\frac{3 \ln 2}{2}\right)+i \frac{3 \pi}{4}\right) c_{\theta} F_{\times}\right] \Theta\left(F_{\text {cut }}-f\right), \\
& \mathcal{C}_{2}^{(4)}=\frac{1}{\sqrt{2}}\left[\left(\frac{113419241}{40642560}+\frac{152987 \nu}{16128}-\frac{11099 \nu^{2}}{1152}+\left(\frac{165194153}{40642560}-\frac{149 \nu}{1792}+\frac{6709 \nu^{2}}{1152}\right) c_{\theta}^{2}+\left(\frac{1693}{2016}-\frac{5723 \nu}{2016}+\frac{13 \nu^{2}}{12}\right) c_{\theta}^{4}\right.\right. \\
& \left.+\left(-\frac{1}{24}+\frac{5 \nu}{24}-\frac{5 \nu^{2}}{24}\right) c_{\theta}^{6}\right) F_{+}+i\left(\left(\frac{114020009}{20321280}+\frac{133411 \nu}{8064}-\frac{7499 \nu^{2}}{576}\right) c_{\theta}\right. \\
& \left.+\left(\frac{5777}{2520}-\frac{5555 \nu}{504}+\frac{34 \nu^{2}}{3}\right) c_{\theta}^{3}+\left(-\frac{1}{4}+\frac{5 \nu}{4}-\frac{5 \nu^{2}}{4}\right) c_{\theta}^{5}\right) F_{\times} \\
& \left.+\nu\left(\frac{721}{96}\left(\left(\chi_{s} \cdot \hat{\mathbf{L}}_{\mathrm{N}}\right)^{2}-\left(\chi_{a} \cdot \hat{\mathbf{L}}_{\mathrm{N}}\right)^{2}\right)-\frac{439}{96}\left(\chi_{s}^{2}-\chi_{a}^{2}\right)\right)\left(\left(1+c_{\theta}^{2}\right) F_{+}+i 2 c_{\theta} F_{\times}\right)\right] \Theta\left(2 F_{\text {cut }}-f\right) \\
& \mathcal{C}_{3}^{(4)}=\frac{1}{\sqrt{3}} s_{\theta} \delta\left[\left(\frac{9 \pi}{8}+i\left(-\frac{189}{40}+\frac{27 \ln (3 / 2)}{4}\right)\right)\left(\left(1+c_{\theta}^{2}\right) F_{+}+i 2 c_{\theta} F_{\times}\right)\right] \Theta\left(3 F_{\text {cut }}-f\right) \\
& \mathcal{C}_{4}^{(4)}=\frac{1}{2}\left[\left(\frac{16109}{2520}-\frac{13367 \nu}{504}+\frac{39 \nu^{2}}{2}+\left(\frac{16}{15}-\frac{16 \nu}{3}+\frac{16 \nu^{2}}{3}\right) c_{\theta}^{2}\left(c_{\theta}^{4}-3\right)+\left(-\frac{10733}{2520}+\frac{7991 \nu}{504}-\frac{53 \nu^{2}}{6}\right) c_{\theta}^{4}\right) F_{+}\right. \\
& +i\left(\left(\frac{2953}{252}-\frac{12023 \nu}{252}+\frac{101 \nu^{2}}{3}\right) c_{\theta}+\left(-\frac{18797}{1260}+\frac{16055 \nu}{252}-\frac{149 \nu^{2}}{3}\right) c_{\theta}^{3}\right. \\
& \left.\left.+\left(\frac{16}{5}-16 \nu+16 \nu^{2}\right) c_{\theta}^{5}\right) F_{\times}\right] \Theta\left(4 F_{\text {cut }}-f\right) \\
& \mathcal{C}_{6}^{(4)}=\frac{1}{\sqrt{6}} s_{\theta}^{4}\left[\left(-\frac{81}{40}+\frac{81 \nu}{8}-\frac{81 \nu^{2}}{8}\right)\left(\left(1+c_{\theta}^{2}\right) F_{+}+i 2 c_{\theta} F_{\times}\right)\right] \Theta\left(6 F_{\text {cut }}-f\right) \\
& \mathcal{C}_{1}^{(5)}=s_{\theta} \delta\left[\left(\frac{37533829}{325140480}+\frac{76171 \nu}{43008}-\frac{8407 \nu^{2}}{4608}+c_{\theta}^{2}\left(-\frac{29850823}{325140480}+\frac{56543 \nu}{129024}+\frac{139 \nu^{2}}{576}\right)\right.\right. \\
& \left.+c_{\theta}^{4}\left(\frac{255}{14336}-\frac{2659 \nu}{64512}+\frac{127 \nu^{2}}{9216}\right)+c_{\theta}^{6}\left(-\frac{1}{9216}+\frac{\nu}{2304}-\frac{\nu^{2}}{3072}\right)\right) F_{+}
\end{aligned}
$$




$$
\begin{aligned}
& +i\left(c_{\theta}\left(-\frac{3453823}{54190080}+\frac{163015 \nu}{64512}-\frac{4237 \nu^{2}}{2304}\right)+c_{\theta}^{3}\left(\frac{34373}{322560}-\frac{11755 \nu}{32256}+\frac{631 \nu^{2}}{2304}\right)\right. \\
& \left.\left.+c_{\theta}^{5}\left(-\frac{7}{4608}+\frac{7 \nu}{1152}-\frac{7 \nu^{2}}{1536}\right)\right) F_{\times}\right] \Theta\left(F_{\text {cut }}-f\right), \\
& \mathcal{C}_{2}^{(5)}=\frac{1}{\sqrt{2}}\left[\left(\frac{85 \pi}{64}(1-4 \nu)+i\left(-\frac{9}{5}+32 \nu\right)+c_{\theta}^{2}\left(\frac{85 \pi}{64}(1-4 \nu)+i \frac{14}{5}(1+4 \nu)\right)+i c_{\theta}^{4} \frac{7}{5}(1-4 \nu)\right) F_{+}\right. \\
& \left.+\left(c_{\theta}\left(2-\frac{282 \nu}{5}+i \frac{85 \pi}{32}(1-4 \nu)\right)+c_{\theta}^{3}\left(-\frac{22}{5}+\frac{94 \nu}{5}\right)\right) F_{\times}\right] \Theta\left(2 F_{\text {cut }}-f\right), \\
& \mathcal{C}_{3}^{(5)}=\frac{1}{\sqrt{3}} s_{\theta} \delta\left[\left(-\frac{8781361}{7225344}-\frac{366781 \nu}{17920}+\frac{15193 \nu^{2}}{1280}+c_{\theta}^{2}\left(-\frac{238136057}{36126720}+\frac{37829 \nu}{71680}-\frac{7073 \nu^{2}}{1280}\right)\right.\right. \\
& \left.+c_{\theta}^{4}\left(-\frac{328347}{143360}+\frac{396009 \nu}{71680}-\frac{10557 \nu^{2}}{5120}\right)+c_{\theta}^{6}\left(\frac{729}{5120}-\frac{729 \nu}{1280}+\frac{2187 \nu^{2}}{5120}\right)\right) F_{+} \\
& +i\left(c_{\theta}\left(-\frac{63633869}{18063360}-\frac{89609 \nu}{2560}+\frac{697 \nu^{2}}{40}\right)+c_{\theta}^{3}\left(-\frac{508689}{71680}+\frac{812727 \nu}{35840}-\frac{4707 \nu^{2}}{320}\right)\right. \\
& \left.\left.+c_{\theta}^{5}\left(\frac{1701}{2560}-\frac{1701 \nu}{640}+\frac{5103 \nu^{2}}{2560}\right)\right) F_{\times}\right] \Theta\left(3 F_{\text {cut }}-f\right), \\
& \mathcal{C}_{4}^{(5)}=\frac{1}{2} s_{\theta}^{2}\left[\left(\frac{8 \pi}{3}(3 \nu-1)+i\left(\frac{56}{5}-\frac{1193 \nu}{30}+\frac{32 \ln 2}{3}(3 \nu-1)\right)\right)\left(\left(1+c_{\theta}^{2}\right) F_{+}+i 2 c_{\theta} F_{\times}\right)\right] \Theta\left(4 F_{\text {cut }}-f\right)(\mathrm{D} 19) \\
& \mathcal{C}_{5}^{(5)}=\frac{1}{\sqrt{5}} s_{\theta} \delta\left[\left(-\frac{854375}{86016}+\frac{3919375 \nu}{129024}-\frac{160625 \nu^{2}}{9216}+c_{\theta}^{2}\left(\frac{40625}{9216}-\frac{40625 \nu}{2304}+\frac{40625 \nu^{2}}{3072}\right)\right.\right. \\
& \left.+c_{\theta}^{4}\left(\frac{1863125}{258048}-\frac{2519375 \nu}{129024}+\frac{85625 \nu^{2}}{9216}\right)+c_{\theta}^{6}\left(-\frac{15625}{9216}+\frac{15625 \nu}{2304}-\frac{15625 \nu^{2}}{3072}\right)\right) F_{+} \\
& +i\left(c_{\theta}\left(-\frac{2388125}{129024}+\frac{3569375 \nu}{64512}-\frac{141875 \nu^{2}}{4608}\right)+c_{\theta}^{3}\left(\frac{3000625}{129024}-\frac{1598125 \nu}{21504}+\frac{51875 \nu^{2}}{1152}\right)\right. \\
& \left.\left.+c_{\theta}^{5}\left(-\frac{21875}{4608}+\frac{21875 \nu}{1152}-\frac{21875 \nu^{2}}{1536}\right)\right) F_{\times}\right] \Theta\left(5 F_{\text {cut }}-f\right), \\
& \mathcal{C}_{7}^{(5)}=\frac{1}{\sqrt{7}} s_{\theta}^{5} \delta\left[\left(\frac{117649}{46080}-\frac{117649 \nu}{11520}+\frac{117649 \nu^{2}}{15360}\right)\left(\left(1+c_{\theta}^{2}\right) F_{+}+i 2 c_{\theta} F_{\times}\right)\right] \Theta\left(7 F_{\text {cut }}-f\right)
\end{aligned}
$$

[1] http://www.ligo.caltech.edu.

[2] http://www.virgo.infn.it

[3] http://www.geo600.uni-hannover.de

[4] http://lisa.jpl.nasa.gov

[5] B. Allen et al., Phys. Rev. Lett. 83, 1498 (1999).

[6] B. Abbott et al. (LIGO Scientific Collaboration), Phys. Rev. D 72, 082001 (2005).

[7] B. Abbott et al. (LIGO Scientific Collaboration), Phys. Rev. D 73, 062001 (2006).

[8] L. Blanchet, Living Rev. Rel. 9, 4 (2006).

[9] A. Buonanno and T. Damour, Phys. Rev. D 62, 064015 (2000).

[10] A. Buonanno, Y. Chen, Y. Pan, H. Tagoshi, and M. Vallisneri, Phys. Rev. D 72, 084027 (2005).

[11] K. Belczynski, R. E. Taam, E. Rantsiou, and M. van der Sluys, Astrophys. J. 682, 474 (2007).

[12] A. Buonanno, Y. Chen, and M. Vallisneri, Phys. Rev. D 67, 104025 (2003), erratum-ibid. D 74, 029904(E) (2006).

[13] C. Cutler and E. Flanagan, Phys. Rev. D 49, 2658 (1994).

[14] E. Poisson and C. Will, Phys. Rev. D 52, 848 (1995).

[15] M. V. van der Sluys et al. (2007), arXiv:0710.1897 [astro-ph]. 
[16] Y. Pan, A. Buonanno, Y. Chen, and M. Vallisneri, Phys. Rev. D 69, 104017 (2004), erratum-ibid. D 74, 029905(E) (2006).

[17] A. Buonanno, Y. Chen, Y. Pan, and M. Vallisneri, Phys. Rev. D 70, 104003 (2004), erratum-ibid. D 74, 029902(E) (2006).

[18] A. Buonanno, Y. Chen, and T. Damour, Phys. Rev. D 74, 104005 (2006).

[19] L. Blanchet, T. Damour, and B. R. Iyer, Phys. Rev. D 51, 5360 (1995).

[20] L. Blanchet, T. Damour, B. R. Iyer, C. M. Will, and A. G. Wiseman, Phys. Rev. Lett. 74, 3515 (1995).

[21] L. Blanchet, Phys. Rev. D 54, 1417 (1996), erratum-ibid. D 71, 129904(E) (2005).

[22] L. Blanchet, G. Faye, B. R. Iyer, and B. Joguet, Phys. Rev. D 65, 061501(R) (2002), erratum-ibid D. 71, 129902(E) (2005).

[23] L. Blanchet, T. Damour, G. Esposito-Farèse, and B. R. Iyer, Phys. Rev. Lett. 93, 091101 (2004).

[24] L. Blanchet, B. R. Iyer, C. M. Will, and A. G. Wiseman, Class. Quantum Grav. 13, 575 (1996).

[25] K. G. Arun, L. Blanchet, B. R. Iyer, and M. S. S. Qusailah, Class. Quantum Grav. 21, 3771 (2004), erratum-ibid. 22, 3115 (2005).

[26] L. E. Kidder, L. Blanchet, and B. R. Iyer, Class. Quant. Grav. 24, 5307 (2007).

[27] L. E. Kidder, Phys. Rev. D 77, 044016 (2007).

[28] L. Blanchet, G. Faye, B. R. Iyer, and S. Sinha, Class. Quantum Grav. 25, 165003 (2008).

[29] G. Faye, L. Blanchet, and A. Buonanno, Phys. Rev. D 74, 104033 (2006).

[30] L. Blanchet, A. Buonanno, and G. Faye, Phys. Rev. D 74, 104034 (2006), erratum-ibid. D 75, 049903 (E) (2007).

[31] L. Kidder, C. Will, and A. Wiseman, Phys. Rev. D 47, R4183 (1993).

[32] B. Mikóczi, M. Vasúth, and L. Á. Gergely, Phys. Rev. D 71, 124043 (2005).

[33] E. Racine, A. Buonanno, and L. Kidder (2008), in preparation.

[34] L. Kidder, Phys. Rev. D 52, 821 (1995).

[35] C. Will and A. Wiseman, Phys. Rev. D 54, 4813 (1996).

[36] B. Owen, H. Tagoshi, and A. Ohashi, Phys. Rev. D 57, 6168 (1998).

[37] R. A. Porto and I. Z. Rothstein, Phys. Rev. Lett. 97, 021101 (2006).

[38] R. A. Porto and I. Z. Rothstein (2007), arXiv:0712.2032[gr-qc].

[39] R. A. Porto and I. Z. Rothstein (2008), arXiv:0802.0720 [gr-qc].

[40] M. Levi (2008), arXiv:0802.1508 [gr-qc].

[41] J. Steinhoff, S. Hergt, and G. Schaefer, Phys. Rev. D 77, 081501(R) (2008).

[42] J. Steinhoff, G. Schaefer, and S. Hergt, Phys. Rev. D 77, 104018 (2008).

[43] J. Steinhoff, S. Hergt, and G. Schafer (2008), arXiv:0809.2200[gr-qc].

[44] R. A. Porto and I. Z. Rothstein (2008), arXiv:0804.0260 [astro-ph].

[45] A. M. Sintes and A. Vecchio, in Rencontres de Moriond:Gravitational waves and experimental gravity, edited by J. Dumarchez (Frontires, Paris, 2000), gr-qc/0005058.

[46] C. Van Den Broeck, Class. Quantum Grav. 23, L51 (2006).

[47] C. Van Den Broeck and A. Sengupta, Class. Quantum Grav. 24, 155 (2007).

[48] C. Van Den Broeck and A. S. Sengupta, Class. Quantum Grav. 24, 1089 (2007).

[49] A. M. Sintes and A. Vecchio, in Third Amaldi conference on Gravitational Waves, edited by S. Meshkov (American Institute of Physics Conference Series, 2000), p. 403, gr-qc/0005059.

[50] T. A. Moore and R. W. Hellings, Phys. Rev. D 65, 062001 (2002).

[51] R. W. Hellings and T. A. Moore, Class. Quant. Grav. 20, S181 (2003).

[52] K. G. Arun, B. R. Iyer, B. S. Sathyaprakash, and S. Sinha, Phys. Rev. D 75, 124002 (2007).

[53] K. G. Arun, B. R. Iyer, B. S. Sathyaprakash, S. Sinha, and C. Van Den Broeck, Phys. Rev. D 76, 104016 (2007).

[54] M. Trias and A. M. Sintes, Phys. Rev. D 77, 024030 (2008).

[55] S. Babak, M. Hannam, S. Husa, and B. Schutz (2008), arXiv:0806.1591 [gr-qc].

[56] A. Vecchio, Phys. Rev. D 70, 042001 (2004).

[57] R. N. Lang and S. A. Hughes, Phys. Rev. D 74, 122001 (2006), erratum-ibid. D 75, 089902 (2007).

[58] R. N. Lang and S. A. Hughes, Astrophys. J. 677, 1184 (2007).

[59] J. Majár and M. Vasúth, Phys. Rev. D 77, 104005 (2008).

[60] A. Buonanno, G. B. Cook, and F. Pretorius, Phys. Rev. D 75, 124018 (2007).

[61] E. Berti, V. Cardoso, J. A. Gonzalez, U. Sperhake, M. Hannam, S. Husa, and B. Brügmann, Phys. Rev. D 76, 064034 (2007).

[62] J. G. Baker, J. R. van Meter, S. T. McWilliams, J. Centrella, and B. J. Kelly, Phys. Rev. Lett. 99, 181101 (2007).

[63] A. Buonanno, Y. Pan, J. G. Baker, J. Centrella, B. J. Kelly, S. T. McWilliams, and J. R. van Meter, Phys. Rev. D 76, 104049 (2007).

[64] M. Hannam, S. Husa, U. Sperhake, B. Brugmann, and J. A. Gonzalez, Phys. Rev. D 77, 044020 (2008).

[65] M. Boyle et al., Phys. Rev. D 76, 124038 (2007).

[66] M. Hannam, S. Husa, B. Brügmann, and A. Gopakumar (2007), arXiv:0712.3787 [gr-qc].

[67] T. Damour and A. Nagar, Phys. Rev. D 77, 024043 (2008).

[68] T. Damour, A. Nagar, E. N. Dorband, D. Pollney, and L. Rezzolla, Phys. Rev. D 77, 084017 (2008).

[69] T. Damour, A. Nagar, M. Hannam, S. Husa, and B. Brügmann, Phys. Rev. D 78, 044039 (2008).

[70] M. Boyle, A. Buonanno, L. E. Kidder, A. H. Mroué, Y. Pan, H. P. Pfeiffer, and M. A. Scheel (2008), arXiv:0804.4184 [gr-qc].

[71] E. Berti, V. Cardoso, J. A. González, U. Sperhake, and B. Brügmann, Class. Quant. Grav. 25, 114035 (2008).

[72] P. Ajith, S. Babak, Y. Chen, M. Hewitson, B. Krishnan, A. M. Sintes, J. T. Whelan, B. Brügmann, P. Diener, N. Dorband, 
et al., Phys. Rev. D 77, 104017 (2008).

[73] Y. Pan, A. Buonanno, J. G. Baker, J. Centrella, B. J. Kelly, S. T. McWilliams, F. Pretorius, and J. R. van Meter, Phys. Rev. D 77, 024014 (2008).

[74] L. Finn and D. Chernoff, Phys. Rev. D 47, 2198 (1993).

[75] E. Poisson, Phys. Rev. D 57, 5287 (1998).

[76] T. A. Apostolatos, C. Cutler, G. J. Sussman, and K. S. Thorne, Phys. Rev. D 49, 6274 (1994).

[77] J. N. Goldberg, A. J. Macfarlane, E. T. Newman, F. Rohrlich, and E. C. G. Sudarshan, J. Math. Phys. 8, 2155 (1967).

[78] L. Landau and E. Lifshitz, Quantum Mechanics (Pergamon, Oxford, 1977).

[79] L. Gualtieri, E. Berti, V. Cardoso, and U. Sperhake, Class. Quantum Grav. 805 (2008).

[80] M. Campanelli, C. O. Lousto, H. Nakano, and Y. Zlochower (2008), arXiv:0808.0713 [gr-qc].

[81] E. Racine, Phys. Rev. D 78, 044021 (2008).

[82] P. Jaranowski and G. Schäfer, Phys. Rev. D 60, 124003 (1999).

[83] P. Jaranowski and G. Schäfer, Ann. Phys. (Berlin) 9, 378 (2000).

[84] V. C. de Andrade, L. Blanchet, and G. Faye, Class. Quantum Grav. 18, 753 (2001).

[85] L. Blanchet and G. Faye, Phys. Rev. D 63, 062005 (2001).

[86] T. Damour, P. Jaranowski, and G. Schäfer, Phys. Lett. 513B, 147 (2001).

[87] K. G. Arun, B. R. Iyer, B. S. Sathyaprakash, and P. A. Sundararajan, Phys. Rev. D 71, 084008 (2005), erratum-ibid. D 72, 069903 (2005).

[88] E. K. Porter and N. J. Cornish (2008), arXiv:0804.0332 [gr-qc].

[89] E. Berti, A. Buonanno, and C. M. Will, Phys. Rev. D 71, 084025 (2005).

[90] C. Cutler, Phys. Rev. D 57, 7089 (1998).

[91] M. Campanelli, C. O. Lousto, and Y. Zlochower, Phys. Rev. D 74, 041501 (2006).

[92] M. Campanelli, C. O. Lousto, and Y. Zlochower, Phys. Rev. D 74, 084023 (2006).

[93] M. Campanelli, C. O. Lousto, Y. Zlochower, B. Krishnan, and D. Merritt, Phys. Rev. D 75, 064030 (2007).

[94] F. Herrmann, I. Hinder, D. Shoemaker, P. Laguna, and R. A. Matzner, Astrophys. J. 661, 430 (2007).

[95] F. Herrmann, I. Hinder, D. M. Shoemaker, P. Laguna, and R. A. Matzner, Phys. Rev. D 76, 084032 (2007).

[96] K. Alvi, Phys. Rev. D 64, 104020 (2001). 